\title{
Psychometric Methods for
}

Automated Test Design

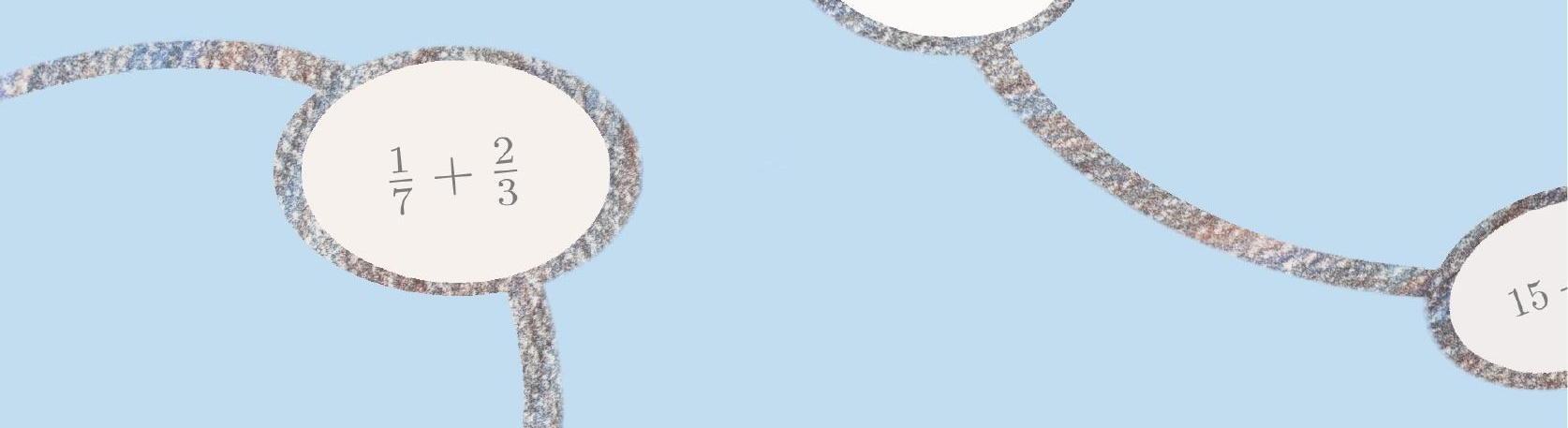



Psychometric Methods for Automated Test Design

Hanneke Geerlings 
Graduation committee

Chairman Prof. Dr. K. I. van Oudenhoven-van der Zee

Promotores Prof. Dr. C. A. W. Glas

Prof. Dr. W. J. van der Linden

Referee Dr. Ir. H. J. A. op den Akker

Members Dr. Ir. G. J. A. Fox

Prof. Dr. H. J. A. Hoijtink

Prof. Dr. H. D. Holling

Prof. Dr. J. K. Vermunt

Prof. Dr. Ing. W. B. Verwey

ISBN: 978-90-365-3330-0

Printed by Ipskamp Drukkers, Enschede

Cover designed by Tessa Vos

Copyright (C)2012 H. Geerlings

This research was partially supported by the Deutsche Forschungsgemeinschaft (grant number HO 1286/5-1). 


\title{
Psychometric Methods for Automated Test Design
}

\author{
DISSERTATION
}

to obtain

the degree of doctor at the University of Twente, on the authority of the rector magnificus, prof. dr. H. Brinksma,

on account of the decision of the graduation committee, to be publicly defended

on Friday, March $23^{\text {rd }}, 2012$ at 14:45

by

Hanneke Geerlings

born on July $3^{\text {rd }}, 1983$

in Oss, The Netherlands 
This dissertation has been approved by the promotores:

Prof. Dr. C. A. W. Glas

Prof. Dr. W. J. van der Linden 


\section{Acknowledgements}

This thesis contains the results of my PhD project at the department of Research Methodology, Measurement and Data Analysis of the University of Twente. I would like to express my gratitude to my colleagues for the pleasant working atmosphere and thank everyone who contributed to this thesis in one way or the other. Special thanks go to my supervisors, Cees Glas and Wim van der Linden, for our discussions and the freedom they gave me in the realization of this thesis. Their suggestions and feedback have been invaluable for my education and the completion of this thesis.

During the project, I have also had the opportunity to work together with researchers from other departments and universities. Specifically, I would like to thank Peter Tellegen and Jacob Laros for their permission to use the dataset on the SON-R $51 / 2-17$ nonverbal intelligence test for Chapter 2 and for the collaboration on Chapter 3; Nina Zeuch, Heinz Holling and their colleagues for the development and testing of the statistical word problems analyzed in Chapter 4; and Roan Boer Rookhuiszen, Rieks op den Akker and Mariët Theune for the development of an item generator for the word problems. Also, I would like to thank the graduation committee for their willingness to read and judge the manuscript.

I am also very grateful for the support of my family and friends. I thank Marjolein Zocca and Tessa Vos for assisting me during the defense as paranymphs. Tessa also created the cover for this thesis, for which I am grateful as well. Finally, I would like to thank Peter Oost, not only for his practical help, but more importantly also for the great time we have together.

Hanneke Geerlings

Enschede, February 2012 



\section{Contents}

1 Introduction 1

1.1 Scope of the Project . . . . . . . . . . . . . . . . . . . . 1

1.2 Automated Assessment . . . . . . . . . . . . . . . 2

1.3 Thesis Overview ....................... 11

2 Modeling Rule-Based Item Generation 13

2.1 Introduction . . . . . . . . . . . . . . . . . . . . . . . 13

2.2 Modeling Approach . . . . . . . . . . . . . . . . . 15

2.2.1 Response Model . . . . . . . . . . . . . . . 16

2.2 .2 Parameter Estimation . . . . . . . . . . . . . 18

2.3 Empirical Study . . . . . . . . . . . . . . . . . . . . . . . . . . . . . . . . . .

2.3.1 Item Structures . . . . . . . . . . . . . . . . . 20

2.3.2 Model Comparison and Model Fit . . . . . . . . . . . 21

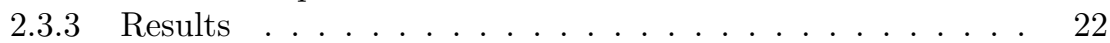

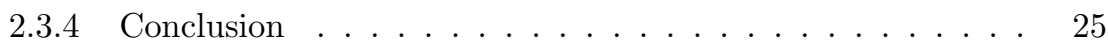

2.4 Simulation Study . . . . . . . . . . . . . . . . . . . . 28

2.4.1 Study Setup. . . . . . . . . . . . . . . . . 28

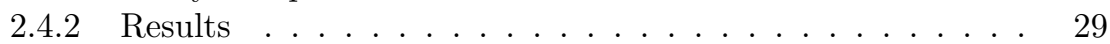

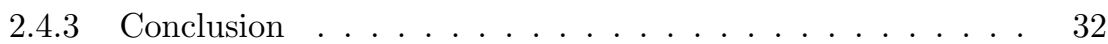

2.5 Discussion . . . . . . . . . . . . . . . . . . . . 32

3 Testing Construction Rules for Intelligence Tests 35

3.1 Introduction . . . . . . . . . . . . . . . . . 35

3.2 Modeling Approach . . . . . . . . . . . . . . . . 36

3.2.1 Response Models . . . . . . . . . . . . . . . . 37

3.2.2 Parameter Estimation . . . . . . . . . . . . . . . 38

3.2.3 Model Fit Assessment . . . . . . . . . . . . . . . . . 39

3.3 Empirical Study . . . . . . . . . . . . . . . . . . . 40

3.3 .1 Item Structures . . . . . . . . . . . . . . . . . . . 42

3.3.2 Results .................... . . 43

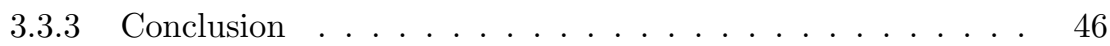

3.4 Simulation Study . . . . . . . . . . . . . . . . . . . . 46

3.4.1 Study Setup. . . . . . . . . . . . . . 49

3.4 Results ..................... 50

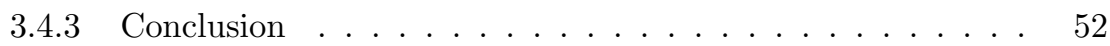

3.5 Discussion ......................... 56 
4 Assessing Item-Family Model Fit $\quad \mathbf{5 7}$

4.1 Introduction . . . . . . . . . . . . . . . . . . 57

4.2 Modeling Approach . . . . . . . . . . . . . . . . . 58

4.2.1 Response Models . . . . . . . . . . . . . . . 58

4.2.2 Parameter Estimation . . . . . . . . . . . . . . 60

4.2 .3 Model Fit Assessment . . . . . . . . . . . . . . . . . . 61

4.2 .4 Response Functions ................ 63

4.3 Empirical Study . . . . . . . . . . . . . . . . . . 63

4.3.1 Dataset .................. 66

4.3.2 Design Matrices and Models . . . . . . . . . . . . 66

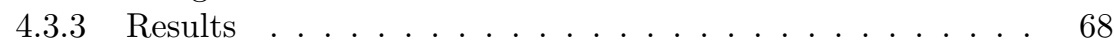

4.4 Discussion . . . . . . . . . . . . . . . . . . 79

5 Optimal Test Design With Rule-Based Item Generation 81

5.1 Introduction . . . . . . . . . . . . . . . . . . . 81

5.2 Rule-Based Item Generation . . . . . . . . . . . . . . . . . . . . . . . 83

5.3 Modeling Rule-Based Item Generation . . . . . . . . . . . . . . . . . 84

5.4 Three Cases of Automated Test Design . . . . . . . . . . . . . 85

5.4.1 Family Information Function . . . . . . . . . . . 86

5.4.2 Test Assembly from Pre-Generated Item Pools . . . . . . . 87

5.4 .3 Test Generation On The Fly . . . . . . . . . . . . 88

5.4.4 Test Generation On The Fly Using Radicals Only . . . . . 89

5.5 Effect of the Covariance Matrix on Family Information . . . . . . . 90

5.6 Simulation Study . . . . . . . . . . . . . . . . . . . . . . . . 92

5.6 .1 Study Setup . . . . . . . . . . . . . . . . . 93

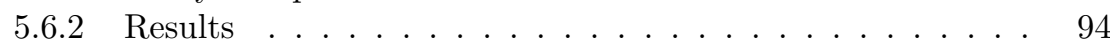

5.7 Discussion . . . . . . . . . . . . . . . . . . . . . 95

\section{Appendices}

A Models and Gibbs Sampling Algorithms 103

A.1 Identical Siblings Models . . . . . . . . . . . . . . . . . 104

A.2 Item Cloning Models . . . . . . . . . . . . . . . . . . . 106

$\begin{array}{lr}\text { B Family Information } & 109\end{array}$

B.1 Identical Siblings Models . . . . . . . . . . . . . . . . . . . . . . . . 109

B.2 Item Cloning Models . . . . . . . . . . . . . . . . . . . . 109

$\begin{array}{ll}\text { References } & 113\end{array}$

$\begin{array}{lr}\text { Samenvatting (Summary in Dutch) } & 121\end{array}$ 


\section{Chapter 1}

\section{Introduction}

Tests are commonly used to measure such diverse things as an individual's abilities, intelligence, psychological traits, attitudes, health status, and quality of life, to name just a few. Within a test, multiple questions or 'items' are administered to increase the precision of measurement. Also, multiple test forms may have to be developed to avoid prior knowledge of the items in consecutive testing sessions. In some of the cases, the time required to generate all these items by hand is considerable, and an alternative is sought for.

By combining research in the fields of cognitive psychology, psychometrics, and computer science, assessments can be partly or fully automated. Apart from potential time savings as compared to generating items by hand, advantages include the possibility of testing on demand with prevention of item disclosure through unique tests, and validity of the generated tests through standardized item generation rules. Using knowledge of the applied item generation rules an estimate can be made of the psychometric properties of sets of similar items that differ only in surface features, so called item 'families'. In this way, the rules allow for example for the generation of parallel test forms, and tests that are adapted in difficulty to a test takers' ability.

Ensuring that the quality of the tests is not inferior to that of hand-generated tests requires methods to translate the test requirements, such as the goal of the test, and the content and difficulty level of the items, into the item generation rules. In the next sections, the scope of the project and the elements of automated assessment systems will be discussed. The chapter will end with an overview of the remaining chapters.

\subsection{Scope of the Project}

This thesis was written as part of a collaborative research project between the departments of Research Methodology, Measurement, and Data Analysis (University of Twente), Electrical Engineering, Mathematics and Computer Science (University of Twente), and Psychology and Sport Science (University of Münster). The goal of this project was to develop a system that can automatically design and 
generate a test consisting of statistical word problems, based on a set of test requirements.

As part of the project, item generation rules and a large sample of statistical word problems were constructed (Zeuch, 2011). Based on this sample, an item generator called Genpex (Generator for narrative probability exercises; Boer Rookhuiszen, 2011) was developed. The present thesis focuses on psychometric models for such rule-based generated items. In particular, estimation and fit assessment of the models (Chapters 2-4), and optimal test design using the models (Chapter 5) will be discussed. An application of the models to the developed sample of statistical word problems shows their utility in practice (Chapter 4). Moreover, in collaboration with researchers from the department of Psychometrics and Statistics of the University of Groningen and the department of Social and Work Psychology of the University of Brasília, their applicability to intelligence data was investigated as well (Chapters 2 en 3 ).

\subsection{Automated Assessment}

In general, the preparation of an automated assessment system consists of the development of candidate models of item difficulty, estimation of the parameters in the models, and model fit assessment and selection. The selected model and the test specifications serve as input for the operational assessment system. This system should design and generate the test and score the test takers' responses. The responses may also be used to update the initial model parameter estimates. The proposed design of automated assessment systems is shown in Figure 1.1.

Given that each of the steps in Figure 1.1 should be performed with no or little interference from a human test developer, adaptations of existing assessment procedures are required. The challenges for each of these preparative and operational steps will be discussed in turn, and the contribution of the present thesis to some of these steps will be outlined. Whereas the methods discussed can be applied to paper-and-pencil tests as well, the focus will be on the possibilities for computerized educational and intelligence assessments. Where relevant, the statistical word problems that can be generated with Genpex will be used as an example.

\subsubsection{Development of a Model of Item Difficulty}

The automation of item generation requires the identification of item features that, when combined, result in construct-relevant items. In this regard, a distinction can be made between item features that are expected to influence the difficulty of the items (radicals; Irvine, 2002), and those that do not and can be used to create diversity among items of similar difficulty (incidentals; Irvine, 2002). Examples of radicals implemented in Genpex are the types of computations required to solve the statistical word problem. The context story, numerical values and the exact sentences are assumed incidentals.

To develop models of item difficulty, information can be obtained from sources such as test developers, existing items, and cognitive theories in the literature. First, rules by which a human test developer generates items can be made explicit using a think-aloud protocol with the assignment to create items of different 


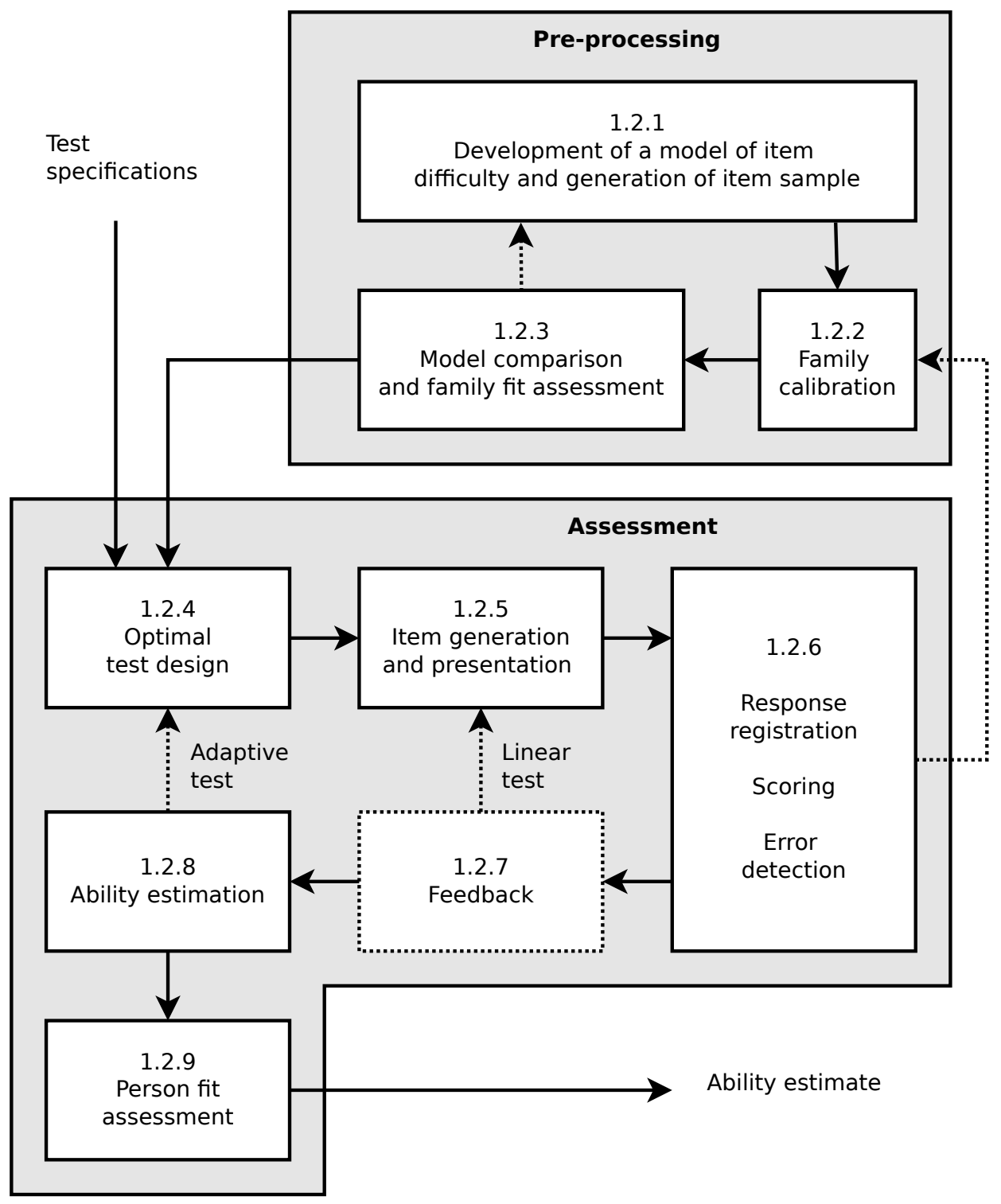

Figure 1.1: Design of an automated assessment system. The numbers refer to the corresponding sections in the text.

difficulty levels. The insights provided by the test developer into the effect of specific item features on the difficulty of items can subsequently be formalized into item-generation rules. Second, item features influencing difficulty can be extracted from a set of hand-made and calibrated items using exploratory data analysis techniques, such as regression trees (Matteucci, Mignani, \& Veldkamp, 2010). In this approach, the difficulty parameters from a standard item response theory (IRT) 
model (for an introduction, see van der Linden \& Hambleton, 1997; Embretson $\&$ Reise, 2000) are regressed on observed item features, and the features that together explain most of the variance in difficulty are selected as potential radicals. Third, research on cognitive theories of problem solving can be used to develop a theory of difficulty. Item difficulty is determined by the number and type of solution steps that are required to solve the item. A second step then has to be performed to define item features that elicit these cognitive processes.

\subsubsection{Family Calibration}

Given a calibration design, to be discussed below, a representative set of items can be generated by means of an application of the radicals and the incidentals (so called rule-based item generation). Data collected on these items can be used to calibrate the parameters of psychometric models. The models serve two purposes. In early stages, they can be used to validate the model of item difficulty, i.e. to verify the categorization of the item features as radicals and incidentals. In later stages, the model that performs best in terms of parsimony and fit to the data can be used to optimally design new tests, and to estimate the ability of new test takers.

In this thesis, several IRT models for use with rule-based item generation will be discussed. The models considered are for dichotomous response variables; that is, each response of a test taker is scored as being either correct or incorrect. In IRT, the effects of test takers and items on the responses are modeled by different sets of parameters. The respective person and item parameters are estimated on the same scale, which enables a comparison of scores even when different test takers have been administered different items. The most basic IRT model, the Rasch model (Rasch, 1960), has only difficulty parameters to describe the items, but extensions have been made to allow for differences in discriminatory power and guessing probabilities of the items (Lord \& Novick, 1968). Three main assumptions of these models are that a single underlying latent trait explains test takers' responses (unidimensionality), that the probability of a correct response increases with ability (monotonicity), and that test takers' responses on the items are independent given the model parameters (local independence).

Models based on radicals and incidentals predict the difficulty of the items to be systematically influenced by the presence of radicals only. Items that have the same set of radicals and thereby form an item family are assumed to have similar psychometric properties. Existing IRT models can be extended to account for the clustering of the items in families. The resulting multilevel models, or item cloning models (Glas \& van der Linden, 2003; Chapter 2), have the advantage of allowing for a generalization to new (uncalibrated) items generated by the same set of radicals and incidentals. In the models, the (transformed) item parameters are modeled by multivariate normal distributions for the families. Within a family, one of the basic IRT models applies, in which each item parameter is equal to its family mean plus an item specific deviation. The (co)variances of the item parameters within families can be modeled either as being heterogeneous or homogeneous across families. In case the deviations are deemed negligible, the models can be simplified by assuming that every item parameter is exactly equal to its family 
mean. The resulting models have been called identical siblings models (Johnson \& Sinharay, 2005; Chapter 4). The effect of the radicals on the difficulty of the items can be investigated by restricting the family difficulties in either of the above mentioned types of models to be equal to a linear combination of radical, and possibly also interaction, effects.

Given the parameters for the families, new items can be designed and test takers' abilities can be estimated based on their responses to these items. Therefore, with a specific psychometric model in mind, the calibration design can be optimized such that collected data provide optimal information on these family parameters given constrained resources. For example, in Section 2.4 the trade-off between the number of items sampled per family and the number of test takers responding to a single item, given a fixed total number of test takers, on the accuracy of the parameter estimates of an item cloning model is investigated. In general, every family that is to be used in the assessment system should be calibrated, and should therefore be represented by a sample of items. However, models with a linear restriction on the family parameters may allow for a more flexible calibration design, because the parameters of the distribution of new uncalibrated families can be computed from the calibrated radical parameters (Section 5.4.4). In this case, each radical should be represented by at least one item, and the radicals should be assigned to the items in a relatively uncorrelated manner to avoid bias in the parameter estimates (Green \& Smith, 1987).

In this thesis, a Bayesian approach to parameter estimation is taken. The multilevel models discussed above are crossed-random effects models, i.e. both the ability parameters and the item parameters are random, and are difficult to estimate in a frequentist framework (see, however, Glas \& van der Linden, 2003, for marginal maximum likelihood estimation of an item cloning model). The Bayesian approach, in particular data-augmented Gibbs sampling (see Appendix A), has the advantage of allowing more complex multilevel models to be estimated using iterative draws from the conditional posterior distribution of subsets of the parameters, which are often in a standard form and therefore easy to sample. It can be shown that, upon convergence, the resulting set of draws are from the joint posterior distribution of the parameters (Casella \& George, 1992).

\subsubsection{Model Comparison and Family Fit Assessment}

As the validity of conclusions based on a psychometric model depends on its fit to the data, it may be worthwhile to consider multiple models and compare their fit. Such a comparison should take into account that an increase in the number of model parameters tends to result in an increased model fit (smaller bias), but may give results that are too sensitive to the specifics of the data (larger inaccuracy). This is well known as the model complexity-model fit trade-off. In information criteria, the trade-off is explicitly taken into account by combining a measure for model fit with a penalty for model complexity. The latter is often based on the number of parameters in the model. In multilevel models, such as the item cloning models discussed above, this number is not easily defined because of the random effects. As an alternative, Spiegelhalter, Best, Carlin, and van der Linde (2002) constructed a measure of model complexity that can be estimated from 
the data. Both their measure of model complexity and model fit are based on deviance statistics. The deviance information criterion (DIC) is defined as the sum of these two measures, and can be used to order different models by preference (Spiegelhalter et al., 2002; Chapter 2).

A more detailed model comparison can be performed by using methods to assess the specific assumptions of the models. A popular Bayesian method in this regard is the posterior predictive check (PPC; Gelman, Meng, \& Stern, 1996; Chapters 3 and 4). PPCs are comparisons of the observed values for a (range of) test statistic(s) with their respective replicated values under the model. The approach is based on the idea that if the model fits well, data replicated under the model are similar to the observed data. Suitable test statistics can be defined based on the data alone or a combination of the data and model parameters. The PPC can be extended to allow for an investigation of the fit of the second level of the item cloning models, i.e. the family distributions for the item parameters. In the extended posterior predictive check (EPPC), the replicated values for the test statistics are computed from replicated data from replicated item parameters drawn under the second-level model (Gelman, Van Mechelen, Verbeke, Heitjan, \& Meulders, 2005; Steinbakk \& Storvik, 2009; Chapter 4).

The test statistics for the (E)PPC should be constructed to reflect questions of interest. Especially important are item and family fit statistics. Questions of interest are whether the hypothesized status of the item features as radicals and incidentals and the assumed (co)variance structure of the item parameters within families is confirmed by the data. Since the sampled items are assumed representative for the families, existing item fit statistics can be used within an (E)PPC to address such questions.

The result of the calibration may be a decision to revise the theory of difficulty and to repeat the previous three steps. Once the final statistical and item generation model has been determined, the item generator can be initialized.

\subsubsection{Optimal Test Design}

The first step in the operational system is to select a design matrix specifying the combinations of radicals that should be employed in the generation of the items, in such a way that a test objective and constraints are satisfied. The incidentals are assumed to be applied randomly.

Because the individual item parameters are unknown (the items have not yet been generated), the objective and constraints of the optimization model should focus on the item families, of which the parameters were estimated using a previous sample of items. Examples of family features that can be used to optimize/constrain family selection are the expected information in the response to a random item from the family (or the expected posterior variance given the response) and the radicals that define the family.

The type of test (linear or adaptive) may also determine the formulation of the optimization model. In a linear test all items are designed prior to test taking. In contrast, in an adaptive test each item is designed during test taking to have optimal properties given an ability estimate based on the previously made items. It has been shown that an adaptive test requires less items to obtain the same 
precision as compared to a non-adaptive test, as items too easy or too difficult (i.e., that do not give much information on the ability of the test taker) are not presented (Weiss \& Kingsbury, 1984).

For the design of linear tests, a popular design objective is to maximize the expected information in the test at various points on the ability scale. For the identical siblings models, the family information measure is similar to the wellknown item information measure (see, for example, van der Linden \& Pashley, 2010, section 1.2.1), with the family parameters substituted for the item parameters. The models, and hence the information measure, ignore the variance in the item parameters within families. For the item cloning models, the uncertainty in the item parameter values is taken into account by integrating them out. Hence, increasing the variance of the item parameters within a family generally decreases the value of the family information measure for this type of models (Chapter 5).

The application of the family information function in the optimal design of tests has a basis in frequentist estimation; the family information function depends on the likelihood only, and its value is the reciprocal of the variance of the maximum likelihood (ML) ability estimate. Hence, a set of families that together give optimal information at the ability parameter will minimize the variance of the ML ability estimate. However, in the design of linear tests, the combination of a maximum information criterion with the expected a posteriori (EAP) ability estimator is popular for practical reasons. Bayesian design criteria are rare for linear tests, and the ML estimator has the disadvantage that it cannot be computed in case of all responses correct or all responses incorrect.

For adaptive testing, van der Linden and Pashley (2010, p. 15) suggest weighting the information function with the posterior distribution of the ability parameter. As an alternative, a minimum expected posterior variance criterion adapted for the case of item-family distributions can be used (Glas \& van der Linden, 2003).

\subsubsection{Item Generation}

Based on the design matrices for the radicals and incidentals, new items can be (semi-)automatically generated. Item generators have been developed previously, for example for:

- arithmetic word problems (Arendasy \& Sommer, 2007),

- cloze items (Smith, Avinesh, \& Kilgarriff, 2010; Liu, Wang, \& Gao, 2005; Mostow et al., 2004; Mitkov, Ha, \& Karamanis, 2006; Goto, Kojiri, Watanabe, Iwata, \& Yamada, 2010; Sumita, Sugaya, \& Yamamoto, 2005),

- and figural matrices (Embretson, 1998; Arendasy \& Sommer, 2005; Hofer, 2004; Freund, Hofer, \& Holling, 2008).

In general, for textual items, such as the statistical word problems discussed above, a distinction is made between concept-to-text generation versus text-to-text generation (Karamanis, Ha, \& Mitkov, 2006), and template-based versus more complex natural language generation (NLG; Reiter \& Dale, 2000). Concept-totext generation refers to a transformation of a non-linguistic representation of the 
information to be expressed to text, whereas in text-to-text generation sentences from digital resources are selected and changed into items. In Genpex, the former approach is used. The 'concept' is the numerical information and the questions that should be included in the item, in the form of formulas. These formulas include all radical information, such as whether the item requires the computation of a conditional probability or a probability of an intersection of independent events.

Template-based text generation requires the (manual) construction of text with open slots that can be filled with sets of alternatives. In the example of a word problem, a template can be a context story for the problem, with slots for the actual numbers that are required to compute an answer, or for synonyms of certain keywords in the problem. In Genpex, templates for basic sentence structures instead of fixed textual templates are used, which can be combined to allow for more complicated structures. Content related information is stored in context files, and is combined with the sentence structures and German grammatical information to obtain a textual representation of the item. Additional 'incidental' variation is obtained by allowing the sentence structures to vary, for example through sentence aggregation, removal of unnecessary words in aggregated sentences (ellipsis), and changes of word order (Boer Rookhuiszen, 2011; Theune, Boer Rookhuiszen, op den Akker, \& Geerlings, 2011).

The items produced by Genpex have an open response format. Whereas multiple choice items have the advantage of simplified response scoring, a disadvantage is the investment required in generating distractors. Distractors should be incorrect but plausible. Another trade-off between the two item types is that whereas multiple choice items provide more information per unit time (Jodoin, 2003), open-ended items may be more authentic to real-world situations (Sireci \& Zenisky, 2006).

An essential part in the development of an item generator is to evaluate whether the items produced essentially have the same quality as expected from a human item writer, especially when the items are not subjected to human review. It should be verified that the system generates items that are linguistically and logically correct and unambiguous. A review of Genpex revealed, for example, that the transformation of certain question formulas into text resulted in items that were ambiguous. Therefore, the software was adapted to exclude the respective question formulas.

\subsubsection{Response Registration, Scoring, and Error Detection}

Computerized testing allows for easy registration of both test taker responses and response times. Whereas this thesis focuses exclusively on the analysis of accuracy ratings, the reader is referred to Klein Entink, Fox, and van der Linden (2009) and Klein Entink, van der Linden, and Fox (2009) for research on IRT models that include both types of output. The added information provided by the response times can result in more accurate estimates of the ability of test takers (van der Linden, Klein Entink, \& Fox, 2010).

In general, two types of methods can be distinguished for the automated scoring of responses. First, some item types lend themselves to an exact comparison 
of the response with a (set of) correct alternative(s). A well-known example is the multiple-choice item type. However, more flexible open response items can also be accommodated by restricting input possibilities through fixed format text fields and on-screen keyboards with only keys that are necessary to solve the item (Bennett, Steffen, Singley, Morley, \& Jacquemin, 1997). The second type of method approximates human ratings by combining extractions of relevant response features into classification decisions (Chapelle \& Chung, 2010; He \& Veldkamp, 2010). Both types of methods require information with regard to what constitutes a (partially) correct response. For the former, the correct alternatives are assumed known, whereas for the latter a training set of human ratings should be available.

Calculation rules in Genpex (Boer Rookhuiszen, 2011) allow the automatic computation of the correct response to a generated item. The rules are, for example, based on distributive, De Morgan, and Bayes' rules. The rules and the correct responses computed by Genpex can be used to develop an automated scoring system that allows for diagnostic, dichotomous, and partial credit scoring. However, both for diagnostic and partial credit scoring, the system should know all relevant alternate solution paths.

Detecting particular student errors may be especially useful in a diagnostic assessment system that contains a feedback component (see Section 1.2.7). The information in the design matrix of the administered items can be used to automatically identify misconceptions of test takers or faults in their problem solving strategy. The incorrect answers of a test taker can be stored and matched with the design matrix. If a test taker responds incorrectly to items with a certain radical, this may indicate that the cognitive processes underlying the radical have not been fully developed. However, as noted by Lee and Corter (2011), such conclusions may be premature, because errors may depend on specific problem contexts or can be occluded by other types of errors (for example, computational). They therefore suggest the use of Bayesian networks, which can be used to compute the probability of specific bugs in a test takers' procedural skills.

\subsubsection{Feedback}

The information from the previous step (responses, accuracy, and possibly the errors detected) can be used to automatically provide a test taker with feedback. In assessment for learning, the most basic form of feedback is whether the given response is correct or incorrect. This feedback can be extended with information on the correct response and the solution steps required to obtain the correct answer. A more complex type of feedback specifically focuses on the errors made by the test taker, and gives information on how these errors are avoided. Through a review of 18 studies, van der Kleij, Timmers, and Eggen (2011) showed that more complex types of feedback, possibly involving remedial information, have the potential to benefit learning.

In a computer-based test, feedback can also be administered adaptively. In case of a correct response, knowing that the answer is correct may be enough. For an incorrect response, the optimal detail and timing of feedback (either immediately after a response or delayed until the end of the test) may depend on the discrepancy between a provisional estimate of student ability and item difficulty. In the context 
of an intelligent tutoring system, Timms (2007) defined three categories based on this discrepancy, and adaptively administered hints with different amounts of detail.

Feedback can also be provided on a test takers' ability to generalize between items from the same family; in other words, to distinguish radicals from incidentals. For example, the ease with which a test taker correctly or incorrectly generalizes between word problems (referred to as the transparency between two problems), depends on whether the solution procedure and the story context of the two problems are the same (Reed, 1987). Two problems are isomorphic if the equations needed to solve the problems are structurally identical. Test takers with a high ability may be more likely to see through surface variation, and recognize whether the underlying solution of two items are the same, whereas test takers with a low ability are more likely to be confused by surface variation.

Note that when test takers learn during the test, their responses are not only dependent on their ability, but also on the order in which the items are administered. In such a case, the assumption of local independence does not hold and additional model parameters are needed to account for the dependency on item administration order (see, for example, Verhelst \& Glas, 1993, Verguts \& De Boeck, 2000, and Glas \& Geerlings, 2009).

\subsubsection{Ability Estimation}

Given the calibrated family parameters, the ability of the test takers can be estimated using an EAP estimator. In the identical siblings models, the item parameters are equated to their family means and the estimate can be computed in the same way as for the respective standard IRT models (Baker \& Kim, 2004, section 7.5.2). In the item cloning models, the parameters of the newly generated and administered items are unknown, which requires an adaptation to the estimator. By integrating over the item parameters, the uncertainty about the unknown item parameter values is taken into account (Glas \& van der Linden, 2003; Chapter 3). Increasing the uncertainty on the item parameters results in a larger standard deviation for the ability estimate. Consequently, to obtain the same precision as for the case of every item having been calibrated separately, more items have to be administered. Or, alternatively, the loss of measurement efficiency can be mitigated by adaptive testing (see Section 1.2.4).

\subsubsection{Person Fit Assessment}

The ability estimate of a test taker is only useful if the model fits the response data of the test taker. Possible reasons for misfit are tiredness or time pressure at the end of the test and guessing or cheating on some of the items. Each of these reasons usually results in inconsistent response behavior during the test. Such inconsistencies can be detected using person fit statistics.

Glas and Meijer (2003) investigated several person fit statistics that have often been used in a frequentist framework for their use in PPCs. The statistics depend on both the data and the model parameters. The approach can be readily applied when an identical siblings model is used, in which the item parameters are assumed 
to be exactly equal to their family means. For the item cloning models, in which only the family distributions are assumed to be known, the statistics can be used in EPPCs (see Section 1.2.3). An advantage of the Bayesian approach is that the uncertainty related to the parameter estimates is explicitly taken into account. Therefore, with increasing uncertainty about the item parameter values, that is, with larger within-family item-parameter variability, the statistics can be expected to have less power to detect person misfit.

\subsection{Thesis Overview}

In this introductory chapter, the design of automated assessment systems was discussed. The focus of the rest of this thesis will be on three of the previously mentioned steps: family calibration, model comparison and family fit assessment (Chapters 2-4), and optimal test design (Chapter 5). The chapters follow a logical order, but have been written to be self contained. Hence, overlap could not be avoided.

The item cloning models with and without radical effect parameters will be further discussed in Chapter 2. In this chapter, hypotheses regarding the radicals and the incidentals are tested by comparing the results of the different models using the DIC, measures of explained variance and pooling (Gelman \& Pardoe, 2006), and Bayesian latent residuals (Fox, 2004; Chaloner \& Brant, 1988). The methodology is illustrated using a dataset on the Analogies subtest of the SON-R $5^{1 / 2-17}$, a non-verbal intelligence test (Laros \& Tellegen, 1991; Tellegen \& Laros, 1993). A parameter recovery study shows the effect of the number of families, items per family, and test takers on the estimation accuracy of the item-level and family-level parameters.

In Chapter 3, a comparison is made between two models with radical effect parameters and equal discrimination parameters: one that allows for residual variance in the item parameters, and one that assumes a perfect prediction. The models are applied to the Mosaics and Patterns subtests of the SON-R 51/2-17, and checked for their fit to the data using PPCs. In a simulation study, the models are compared with regard to the robustness of their model parameters in the presence of unexplained variance.

In Chapter 4, a larger set of identical sibling and item-cloning models is applied to a dataset for statistical word problems (Zeuch, 2011). Assumptions on the item-parameter variability within families, and the prediction of the family discrimination and difficulty parameters by the radicals are tested using both PPCs and EPPCs. The most parsimonious model that fits the data well is selected and its results are visualized using item and family expected response functions.

In Chapter 5, the item cloning models are used to design optimal tests according to a family information measure. The information measure takes the uncertainty about the item parameter values into account and generally yields a lower information value when there is more variation in the item parameter values within a family. Knowledge on item generation rules is used to constrain item family selection. A simulation study shows the effect of different amounts of within-family item-parameter variability and radical constraints on the optimal solution to test design problems. 
The thesis concludes with two appendices. Appendix A presents the Bayesian estimation algorithms for the models of Chapters 2-4. It is shown how a large set of models can be estimated using two general algorithms; one for the identical siblings models and another for the item cloning models. The differences between models within the two classes are identified by the different design matrices for the discrimination, difficulty, and guessing parameters. Appendix B discusses the computations for the family information measures for both types of models. 


\title{
Chapter 2
}

\section{Modeling Rule-Based Item Generation}

\begin{abstract}
An application of a hierarchical IRT model for items in families generated through the application of different combinations of design rules is discussed. Within the families, the items are assumed to differ only in surface features. The parameters of the model are estimated in a Bayesian framework, using a data-augmented Gibbs sampler. An obvious application of the model is computerized algorithmic item generation. Such algorithms have the potential to increase the cost-effectiveness of item generation as well as the flexibility of item administration. The model is applied to data from a non-verbal intelligence test created using design rules. In addition, results from a simulation study conducted to evaluate parameter recovery are presented.
\end{abstract}

Key words: hierarchical modeling, item generation, item response theory, Markov chain Monte Carlo method.

\section{$2.1 \quad$ Introduction}

One of the main reasons why automated item generation has gained interest lately is the need for large item pools to further flexibility in test administration while avoiding overexposure of the items. When done manually, item writing can be a costly and time-consuming endeavor. However, given a well-specified set of rules, a computer can generate a large pool of items in a negligible amount of time. An additional advantage of automated item generation is the availability of precise information about how the items have been constructed. For instance, the information can be used as a check on the validity of the test. In the present article, a hierarchical item response theory (IRT) model incorporating information about the item-design rules is used to analyze a dataset consisting of responses

Adapted from: Geerlings, H., Glas, C. A. W., \& van der Linden, W. J. (2011). Modeling rule-based item generation. Psychometrika, 76, 337-359. 
to rule-based generated items. The parameters of the model are estimated in a Bayesian fashion, using a data-augmented Gibbs sampler.

The model is developed for a combination of two methods of automated item generation. The first method is generation based on cognitive analysis of the item domain. The results from the analysis are then used to devise rules for the generation of new items (Embretson, 1999). Irvine (2002) introduced the term "radicals" to refer to such rules. An example of a radical is whether or not Bayes' rule has to be applied to solve a statistics item. Radicals can be used to automate item generation. In addition, the radicals can be assumed to be important determinants of item difficulty. A psychometric model accounting for the effects of radicals is the linear logistic test model (LLTM; Fischer, 1973; Freund et al., 2008; Holling, Bertling, \& Zeuch, 2009). This model decomposes the difficulty parameter of the Rasch (1960) model into a linear combination of the effects of radicals. An error term can be added to the model to make it less restrictive.

The second method is item cloning. The goal of item cloning is to generate a set or family of items that look different but are generated by the same combination of radicals. The families are created from parent items for the combinations of radicals by changing some of their surface features. Irvine (2002) refers to these features as "incidentals". Incidentals are not assumed to influence the difficulty of the items in any systematic way; their only goal is to ensure that items within a family are sufficiently different to avoid solving them just by remembering earlier solutions. For the earlier example of a statistics item, an incidental could be a context story with information irrelevant to the formal statistical problem. Incidentals can be produced, for example, with the help of replacement sets for some of the insignificant elements of the parent items (Hively, Patterson, \& Page, 1968; Millman \& Westman, 1989; Osburn, 1968; Roid \& Haladyna, 1982), by transforming their text by means of linguistic rules (Bormuth, 1970), or by applying other natural language generation techniques. A psychometric model for this approach is the hierarchical model proposed by Glas and van der Linden (2001, 2003; see also Sinharay, Johnson, \& Williamson, 2003; Glas, van der Linden, \& Geerlings, 2010). This model, which will be referred to as the item cloning model (ICM), assumes that the parameters of the individual items are a combination of family parameters with a random component to allow for the unsystematic variation caused by incidentals. In principle, if the family parameters have been estimated from a previous sample of items with enough precision, newly generated items would not have to be calibrated at all, because their parameters can simply be assumed to be drawn from the known family distributions.

Ideally, a system for automated item generation based on these two methods can produce a large collection of item families. Within each family, similarity among items is caused by the use of the same radicals whereas dissimilarities would be the result of incidentals only. In the present article, we combine the ICM with an LLTM-like structure for the expected value of the item difficulty parameters for each family. The structure decomposes the mean family difficulty into separate effects for each of its radicals.

The model, which will be labeled the linear item cloning model (LICM), is discussed in more detail in the next section. In the third section, an empirical 
study using a dataset from a non-verbal intelligence test is presented to show how the model can be applied in practice. Furthermore, a simulation study was conducted to investigate the effect of different factors in the sampling design on the recovery of the model parameters during calibration. The results from this study will be discussed in the fourth section. The article concludes with a discussion of practical applications and future research on the model.

\subsection{Modeling Approach}

In IRT-based item calibration, person parameters are often considered random to represent the fact that the items are calibrated using data from a random sample of persons. In the current setting, items are treated as random as well, because they can be considered random instantiations ("clones") from their respective families. Therefore, to calibrate item families, it seems natural to model both the person and item parameters as random. The resulting model is a crossed-random effects model. Crossed-random effects models are difficult, but not impossible, to estimate in a frequentistic framework (see Van den Noortgate, De Boeck, \& Meulders, 2003, Glas \& van der Linden, 2003, and Cho \& Rabe-Hesketh, 2011). However, component-wise estimation in the form of Gibbs sampling from the conditional posterior distributions of the parameters reduces the estimation into manageable pieces. Gibbs sampling of the parameters of the ICM has been considered in Glas and van der Linden (2001) and Sinharay et al. (2003). A Gibbs sampler for a similar model, with the correlations between the item parameters restricted to zero, was proposed by Janssen, Tuerlinckx, Meulders, and De Boeck (2000) in the context of criterion-referenced measurement.

In the present article, we do not only wish to account for item-cloning effects in the model, but also for item-generation rules that are hypothesized to have a fixed effect on the item difficulties. The LLTM (Fischer, 1973) was one of the first examples of adding explanatory variables to otherwise descriptive models. The model was later extended to account for residual variance (random-effects LLTM; Janssen, Schepers, \& Peres, 2004) and for random weights (random-weights LLTM; Rijmen \& De Boeck, 2002). Explanatory models have been described in a more general nonlinear mixed modeling framework by Rijmen, Tuerlinckx, De Boeck, and Kuppens (2003), and De Boeck and Wilson (2004).

To summarize, the LICM is a hierarchical IRT model with higher-level explanatory variables accounting for the effects of both item cloning and item generation rules. Fox and Glas (2001) presented a Gibbs sampler for a multilevel model with explanatory variables for the person parameters. In the present article, the focus will be on explanatory variables for the item families. Extending the ICM by Glas and van der Linden (2003) with a linear structure on the family difficulty parameters has several advantages. First, the model can be used to check the theory used to generate the items, and thereby function as a quality control mechanism. In this regard, the model fits into the frameworks of assessment engineering ( $\mathrm{AE}$; Luecht, 2009) and evidence-centered design (ECD; Mislevy \& Levy, 2007). Both frameworks share the point of view of assessment as a process of obtaining evidence about the ability of a test taker. Items developed according to a cognitive model, that is, a model with the cognitive steps a test taker has to take to solve the item, 
can provide such evidence. In doing so, psychometric models are used in a confirmatory manner-to test hypotheses provided by the cognitive model. For instance, methods for model comparison and model fit could be used to investigate whether the radicals properly explain the difficulty of a family, and whether the incidentals only have a random effect on the difficulty of the items (see Section 2.3.2). Second, using the information provided by the item-design process, the estimation of the difficulty of a particular family can borrow strength from data available for the other families (see Section 2.4.2). Finally, the model supports item generation onthe-fly; that is, test administration in which the items are sampled from calibrated families in real time and ability is estimated using the family parameters.

\subsubsection{Response Model}

Consider $f=1, \ldots, F$ item families, with family $f$ consisting of item $i_{f}=1, \ldots I_{f}$. In total, there are $K$ items. The families are identified by combinations of radicals $r=1, \ldots, R$. Each of $n=1, \ldots, N$ persons is administered a subset of the $K$ items, resulting in a response vector with realizations of response variables $U_{i_{f} n}=\{0,1\}$ for every person $n$. Missing responses created by this design are considered as missing at random. Therefore, for convenience, and without loss of generality, we will not make the design explicit in the notation. Furthermore, it will be assumed that each item sampled from a family is administered to more than one test taker. Figure 2.1 offers a simplified representation of an item pool with algorithmically generated items.

\section{First-Level Model}

The first-level model specifies the probability of a person giving a correct response on an item as

$$
p\left(U_{i_{f} n}=1 \mid \theta_{n}, a_{i_{f}}, b_{i_{f}}, \gamma_{i_{f}}\right)=\gamma_{i_{f}}+\left(1-\gamma_{i_{f}}\right) \Phi\left(a_{i_{f}} \theta_{n}-b_{i_{f}}\right) .
$$

This is the three-parameter normal-ogive (3PNO) model in which $a_{i_{f}}, b_{i_{f}}$, and $\gamma_{i_{f}}$ are the item discrimination, difficulty and guessing parameters, respectively, $\theta_{n}$ is the person parameter, and $\Phi($.$) is the cumulative normal density function.$ Alternatively, the $2 \mathrm{PNO}$ (the $3 \mathrm{PNO}$ without the guessing parameter) can be used as the first-level model.

Note that Glas and van der Linden (2003) originally presented the ICM with the three-parameter logistic (3PL) model as the first-level model. Also, the parameterization of the normal ogive model in (2.1) is different from the usual parameterization, which has $a_{i_{f}}\left[\theta_{n}-b_{i_{f}}\right]$ as the argument of $\Phi($.$) . However, the$ normal-ogive link function in combination with the parameterization in (2.1) has the advantage of easy sampling from the conditional posterior distributions (see Appendix A).

\section{Second-Level Model}

The item parameters, denoted as $\boldsymbol{\xi}_{i_{f}}$, are transformed as:

$$
\boldsymbol{\xi}_{i_{f}}=\left(a_{i_{f}}, b_{i_{f}}, \operatorname{logit} \gamma_{i_{f}}\right) .
$$




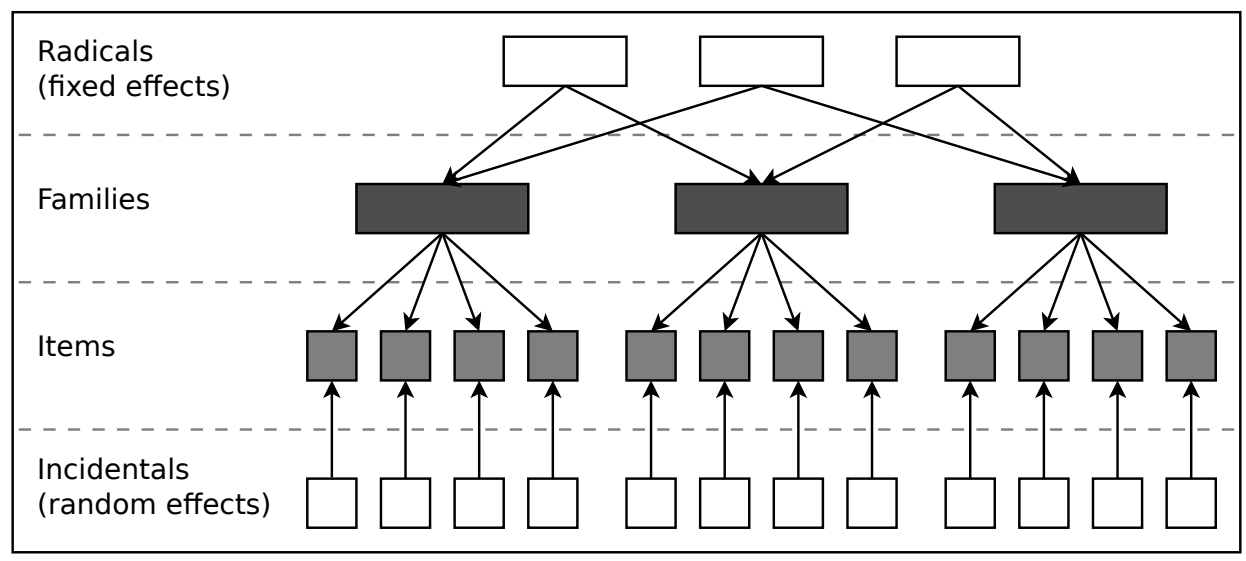

Figure 2.1: The relationship of radicals and incidentals with families and items.

We will use $c_{i_{f}}$ to denote the transformed guessing parameter. Because of this transformation, it can be assumed that the parameters $\boldsymbol{\xi}_{i_{f}}$ have a multivariate normal distribution

$$
\boldsymbol{\xi}_{i_{f}} \sim M V N\left(\boldsymbol{\mu}_{f}, \boldsymbol{\Sigma}_{f}\right)
$$

with $\boldsymbol{\mu}_{f}$ a vector of mean values for the item parameters and $\boldsymbol{\Sigma}_{f}$ the covariance matrix of the item parameters for family $f$.

As an alternative, when the covariance matrices can be assumed to be approximately equal across families, a common covariance matrix can be used,

$$
\boldsymbol{\xi}_{i_{f}} \sim M V N\left(\boldsymbol{\mu}_{f}, \boldsymbol{\Sigma}\right)
$$

The model with family-specific covariance matrices will be labeled LICM-F; the model with a common covariance matrix will be labeled LICM-C. In both models, the mean difficulty of a family is postulated to be a linear combination of the effects of the radicals used to generate an item:

$$
\mu_{b_{f}}=\sum_{r=1}^{R} d_{f r} \beta_{r}
$$

where $\beta_{r}$ is the effect of radical $r$ on the mean difficulty of the item families and $d_{f r}$ is a design variable denoting how often radical $r$ should be used within an item to generate an item from family $f$. Thus, at the item level,

$$
b_{i_{f}}=\sum_{r=1}^{R} d_{f r} \beta_{r}+\varepsilon_{i_{f}}, \quad \varepsilon_{i_{f}} \sim N\left(0, \sigma_{b_{f}}^{2}\right),
$$

with $\sigma_{b_{f}}^{2}$ the second diagonal element of $\boldsymbol{\Sigma}_{f}$. As can be seen from (2.6), the radicals determine the mean family difficulty parameter $\mu_{b_{f}}$ whereas the incidentals determine the family covariance matrix $\boldsymbol{\Sigma}_{f}$.

It is assumed that $\theta$ has a normal distribution with mean $\mu_{\theta}$ and standard deviation $\sigma_{\theta}$. We set $\mu_{\theta}=0$, and $\sigma_{\theta}=1$ to identify the model. 


\subsubsection{Parameter Estimation}

In the studies reported below, the parameters of the model were estimated in a Bayesian framework with data-augmented Gibbs sampling. The specific Gibbs sampling algorithm is described in Appendix A.2 and was programmed in the software environment R (R Development Core Team, 2009).

Independent priors were used for the hyperparameters $\boldsymbol{\lambda}=\left(\boldsymbol{\mu}_{a}, \boldsymbol{\beta}, \boldsymbol{\mu}_{c}\right)$ and $\boldsymbol{\Sigma}_{f}$ (LICM-F) or $\boldsymbol{\Sigma}$ (LICM-C). A convenient prior for $\boldsymbol{\lambda}$ is the multivariate normal distribution with mean $\boldsymbol{\lambda}_{0}$ and covariance matrix $\boldsymbol{V}_{0}$,

$$
\boldsymbol{\lambda} \sim \operatorname{MVN}\left(\boldsymbol{\lambda}_{0}, \boldsymbol{V}_{0}\right)
$$

The prior for $\boldsymbol{\Sigma}_{f}$ was the inverse-Wishart distribution with sum of squares $\boldsymbol{S}_{0}$ and degrees of freedom $\nu_{0}$ greater than or equal to the dimension of $\boldsymbol{\Sigma}_{f}$ (Gelman, Carlin, Stern, \& Rubin, 2004),

$$
\boldsymbol{\Sigma}_{f} \sim \text { inverse-Wishart }\left(\boldsymbol{S}_{0}, \nu_{0}\right)
$$

Let $\boldsymbol{u}=\left(\left(u_{i_{f} n}\right)\right), \boldsymbol{\xi}=\left(\boldsymbol{\xi}_{f}\right)=\left(\left(\boldsymbol{\xi}_{i_{f}}\right)\right)$; the other boldfaced parameters are defined analogously. Combining the above priors with the likelihood results in the following joint posterior for the LICM-F:

$$
\begin{gathered}
p(\boldsymbol{z}, \boldsymbol{w}, \boldsymbol{\theta}, \boldsymbol{\xi}, \boldsymbol{\mu}, \boldsymbol{\Sigma}, \boldsymbol{\lambda} \mid \boldsymbol{u}, \boldsymbol{Q}) \propto \\
\prod_{f=1}^{F}\left[p\left(\boldsymbol{z}, \boldsymbol{w} \mid \boldsymbol{u}, \boldsymbol{\theta}, \boldsymbol{\xi}_{f}\right) p\left(\boldsymbol{\xi}_{f} \mid \boldsymbol{\lambda}, \boldsymbol{Q}_{f}, \boldsymbol{\Sigma}_{f}\right) p\left(\boldsymbol{\Sigma}_{f} \mid \boldsymbol{S}_{0}, \nu_{0}\right)\right] p(\boldsymbol{\theta}) p\left(\boldsymbol{\lambda} \mid \boldsymbol{\lambda}_{0}, \boldsymbol{V}_{0}\right),
\end{gathered}
$$

in which $\boldsymbol{Q}_{f}$ is a design matrix such that $\boldsymbol{\mu}_{f}=\boldsymbol{Q}_{f} \boldsymbol{\lambda}$. The composition of $\boldsymbol{Q}_{f}$ and the data-augmentation variables $\boldsymbol{Z}$ and $\boldsymbol{W}$ are explained in the Appendix. The posterior density of the LICM-C is obtained by replacing $\boldsymbol{\Sigma}_{f}$ in (2.9) by $\boldsymbol{\Sigma}$.

Results from a Markov chain can be used as draws from the full posterior only upon convergence of the chain. In the literature, several convergence diagnostics have been proposed. However, none of them is foolproof, and the use of multiple diagnostics to assess different aspects of the convergence is generally recommended. In the studies below, we used Geweke's (1992), Heidelberger and Welch's (1983) and Raftery and Lewis' (1992) diagnostics to assess convergence. Geweke's (1992) diagnostic is a Z-score test for the equality of the means of the first $10 \%$ and last $50 \%$ of the values drawn in the Markov chain after the burn-in period. Heidelberger and Welch's (1983) and Raftery and Lewis' (1992) diagnostics are based on a criterion of accuracy for the estimated mean and a quantile $q$ of the parameter distributions, respectively. All convergence diagnostics are available in the package Coda for R (Plummer, Best, Cowles, \& Vines, 2006).

\subsection{Empirical Study}

The example is an analysis of the Analogies subtest of the SON-R 51/2-17 nonverbal intelligence test (Laros \& Tellegen, 1991; Tellegen \& Laros, 1993). Each item of the subtest consisted of three different pictures composed of geometrical figures (A, B, and C). The test taker had to choose a fourth picture (D) from a set of four alternatives such that the transformation(s) applied to $\mathrm{C}$ to obtain $\mathrm{D}$ (such 

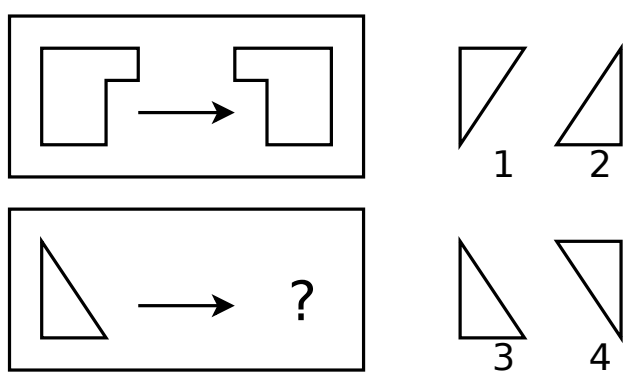

Figure 2.2: Example item similar to the items in the Analogies subtest of the SON-R 51/2-17.

as form changes and rotations) were the same as those applied to A to create B; that is, the test taker had to complete $\mathrm{A}: \mathrm{B}=\mathrm{C}:$ ?. An example item similar to the items in the Analogies subtest of the SON-R 51/2-17 is presented in Figure 2.2. The test was taken by 1,350 children of age $6-14$.

The authors of the test constructed its items by systematically varying their features in accordance with a postulated theory of item difficulty. In all, they distinguished 11 different levels of difficulty and created three items at each level. We interpret this as 11 families with three items each. The difficulty levels were used in an adaptive administration of the test, which ran as follows: The items were combined into three series, where each series contained one item from every family. Within a series, the 11 families were ordered from easy to difficult. Every test taker started with the first item in the first series, and continued with the series until two incorrect answers were given. He or she then continued with item $m$ in the second series, where $m$ was one less than the number of items scored correctly in the first series. The procedure continued similarly, with missing responses at the start of the second series counted as correct responses in the computation of $m$, until two incorrect answers were given to the items in the third series or the last item in this series had been reached. Because of the adaptive nature of the procedure, items too difficult for a particular test taker were not administered.

As guessing was expected not to be an issue, the 11 item families were analyzed using the 2 PNO model as the first-level model. We nevertheless investigated possible item misfit due to guessing by means of a Bayesian latent residual analysis (see Section 2.3.2). As the number of items per family was low, we used the LICM-C. Missing data due to the adaptive design of the test, both at the beginning and the end of the series, can be treated as missing at random. This is justified because the item administration design was completely determined by the observed responses. Because of this, the ignorability principle for missing data (Rubin, 1976; Glas, 2010) holds, and bias in the parameter estimates was avoided.

The total number of observations per item ranged from 139 to 1,350; per family the range was from 680 (Family 11) to 2,483 (Family 5). The prior means of the family discrimination and effect parameters $\boldsymbol{\lambda}_{0}$ were set equal to one. The variances of the prior scale matrix $\boldsymbol{S}_{0}$ were set equal to 0.1 and the covariances to 0.05 . Furthermore, $\boldsymbol{V}_{0}$ was set equal to a diagonal matrix with elements 100 (representing a case of low prior information) and $\nu_{0}$ was set equal to two (i.e., 


$\left[\begin{array}{lll}1 & 1 & 1 \\ 1 & 1 & 1 \\ 1 & 1 & 1 \\ 1 & 1 & 1 \\ 1 & 1 & 2 \\ 1 & 2 & 1 \\ 1 & 1 & 2 \\ 1 & 2 & 2 \\ 1 & 2 & 2 \\ 1 & 2 & 3\end{array}\right]$

Figure 2.3: Design matrix of the restricted model $\boldsymbol{D}_{2}$.

smallest value of $\nu_{0}$ given the dimension of the covariance matrix; Gelman et al., 2004).

Expected a posteriori (EAP) estimates of the hyperparameters of the LICM-C were computed from 100,000 iterations of the Gibbs sampler after the first 20,000 iterations for burn-in. Convergence of the sampler for the hyperparameters was checked using Geweke's (1992) and Heidelberger and Welch's (1983) diagnostics and by inspecting convergence plots.

\subsubsection{Item Structures}

Laros and Tellegen (1991) created the items according to the theory that item difficulty increases with (1) the number of transformations performed on the Aterm, (2) the number of basic elements in the A-term, (3) the complexity of the transformations on the A-term, (4) the dissimilarity between the A-term and the C-term, and (5) the similarity between the correct and incorrect alternatives. For example, to solve the item in Figure 2.2 only one transformation on one basic element is needed (the transformation is to mirror the triangle on the vertical axis). As the item families were not systematically designed with respect to the other three factors (see Laros \& Tellegen, 1991; Tellegen \& Laros, 1993), we tested the hypothesis that the first two rules explained the family difficulty parameters against the alternative that the effects of these other factors could not be ignored.

To test the hypotheses, two models with different design matrices were constructed. The first model contained a different dummy rule for each family; i.e. the design matrix for this baseline model, $\boldsymbol{D}_{1}$, was an identity matrix of size 11 . Note that fitting the LICM for this design matrix is comparable to fitting the ICM by Glas and van der Linden (2003).

The other model was constructed to test the effect of the two rules. For the first ten families, all three items within each family had the same value for the two rules. Family 11 did not systematically vary according to these rules. Therefore, this family was not modeled by the two rules, but by a family-specific intercept. In this way, all eleven families could be analyzed in the same run. As noted above, removing the items from Family 11 from the analysis would have caused a violation of the ignorability principle for missing data.

The design matrix for the first ten families was constructed to investigate the 
effect of the number of transformations and the number of basic elements in the items (see Figure 2.3). The rows of the matrix correspond to the different families and the columns to the different rules, except for the first column, which represents the intercept. The entries in the second and third column represent the effect of adding a specific number of transformations and basic elements, respectively, to the item. Observe that in this model Families 1-4, 5 and 7, and 8-9 were restricted to have the same mean difficulty. However, the means of their discrimination parameters were allowed to vary.

\subsubsection{Model Comparison and Model Fit}

To test the hypotheses mentioned above, the two models were compared by means of the deviance information criterion (DIC; Spiegelhalter et al., 2002). The DIC is a model selection criterion based on a measure of model fit, $\bar{D}(\boldsymbol{\eta})$, and a penalty for model complexity, $p_{D}$ :

$$
D I C=\bar{D}(\boldsymbol{\eta})+p_{D}
$$

where $\boldsymbol{\eta}$ are the parameters of the model. Define the deviance as -2 times the $\log$ likelihood:

$$
D(\boldsymbol{\eta})=-2 \log \prod_{f=1}^{F} p\left(\boldsymbol{u} \mid \boldsymbol{\theta}, \boldsymbol{\xi}_{f}\right) p\left(\boldsymbol{\xi}_{f} \mid \boldsymbol{\mu}_{f}, \boldsymbol{\Sigma}\right) .
$$

Model fit, $\bar{D}(\boldsymbol{\eta})$, is then defined as the posterior mean of the deviance, and model complexity, $p_{D}$, as $\bar{D}(\boldsymbol{\eta})$ minus the deviance at the posterior mean of the parameters $D(\overline{\boldsymbol{\eta}}) . \bar{D}(\boldsymbol{\eta})$ and $D(\overline{\boldsymbol{\eta}})$ can be estimated using posterior simulations of the parameters. The use of the DIC is such that the model with the smallest value for the DIC is to be preferred.

For the LICM, it is important to investigate whether the radicals can properly explain the family difficulty parameters. There are several reasons for a possible discrepancy between the family difficulty parameters in the ICM and LICM. First of all, the set of radicals in the LICM may be incomplete. Similarly, the design matrix may have been misspecified. For example, omitting a term for an interaction between certain radicals that may have occurred may result in bias in the estimated family difficulty parameters. Finally, the assumption of a linear relationship between the radicals and the family difficulty parameters may not hold.

To investigate whether the specified radicals and design matrix could properly explain the family difficulty parameters, a statistic based on a suggestion by one of the reviewers was applied. For each family, the mean and $95 \%$ highest posterior density (HPD) interval of the difference between the empirical and modeled means of the item parameters were computed across iterations of the Gibbs sampler. A HPD interval for this statistic not including zero was taken as a sign of model misspecification. Conclusions based on the statistic may be conservative, because the family mean parameters also serve as means of the prior distributions in the estimation of the item parameters. However, the impact of the means of the family priors is moderated by the estimated covariance matrix, which will be larger when an estimated family difficulty parameter lies further away from the 
empirical mean of the item difficulty parameters. The impact can therefore be expected to automatically decrease with increasing bias in the family parameters.

An alternative way of identifying misfit due to the linear structure on the family difficulty parameters is to compare the ICM and the LICM with regard to the family difficulty estimates, the explained variance at the level of the item parameters, and the degree of pooling of the item parameters around their family means. To this end, Gelman and Pardoe's (2006) explained variance, $R^{2}$, and pooling factor, $\lambda$, were computed. (The standard notation for the pooling factor should not be confused with that for the hyperparameters of the model.) The explained variance in the item difficulty parameters can be computed as

$$
R_{b}^{2}=1-\frac{E\left[\operatorname{Var}\left(b_{i_{f}}-\mu_{b_{f}}\right)\right]}{E\left[\operatorname{Var}\left(b_{i_{f}}\right)\right]},
$$

where $E$ represents the mean over the posterior simulations, and Var represents the finite-sample variance operator over the parameters. The pooling factor of the item difficulty parameters can be computed as

$$
\lambda_{b}=1-\frac{\operatorname{Var}\left[E\left(b_{i_{f}}-\mu_{b_{f}}\right)\right]}{E\left[\operatorname{Var}\left(b_{i_{f}}-\mu_{b_{f}}\right)\right]} .
$$

A pooling factor of less than .5 indicates a higher degree of within-family than between-family information. The explained variance and pooling factor for the discrimination parameters, $R_{a}^{2}$ and $\lambda_{a}$, can be computed analogously.

Bayesian latent residual analyses were performed to further investigate the fit of the first-level models to the data (Fox, 2004; Johnson \& Albert, 1999). For the $2 \mathrm{PNO}$, the Bayesian latent residual corresponding to response $U_{i_{f} n}$ can be defined as

$$
\epsilon_{i_{f} n}=Z_{i_{f} n}-a_{i_{f}} \theta_{n}+b_{i_{f}}
$$

where $Z_{i_{f} n}$ is a data-augmentation variable explained in Appendix A. Outliers were defined as observed responses with absolute residuals greater than two standard deviations. The posterior probabilities of correct or incorrect responses being outliers were computed from the draws of the Markov chain as

$$
\begin{aligned}
& p\left(\left|\epsilon_{i_{f} n}\right|>2 \mid U_{i_{f} n}=1, \theta_{n}, \boldsymbol{\delta}_{i_{f}}\right)=\frac{\Phi(-2)}{\Phi\left(a_{i_{f}} \theta_{n}-b_{i_{f}}\right)}, \\
& p\left(\left|\epsilon_{i_{f} n}\right|>2 \mid U_{i_{f} n}=0, \theta_{n}, \boldsymbol{\delta}_{i_{f}}\right)=\frac{\Phi(-2)}{1-\Phi\left(a_{i_{f}} \theta_{n}-b_{i_{f}}\right)},
\end{aligned}
$$

respectively. A high percentage of outlying responses indicates a poor model fit. In particular, many outliers among the correct responses can be an indication that guessing occurred.

\subsubsection{Results}

Table 2.1 presents the values of the DIC and its constituent model fit measure $\bar{D}(\boldsymbol{\eta})$, model complexity measure $p_{D}$, the explained variance $R^{2}$ and pooling factor $\lambda$ for the two models. As indicated by its value for $p_{D}$, ignoring the residual 
Table 2.1: Summary statistics (DIC, $\bar{D}(\boldsymbol{\eta}), p_{D}, R^{2}$, and $\lambda$ ) for the two models.

\begin{tabular}{lrrrrrrr}
\hline Model & DIC & $\bar{D}(\boldsymbol{\eta})$ & $p_{D}$ & $R_{a}^{2}$ & $\lambda_{a}$ & $R_{b}^{2}$ & $\lambda_{b}$ \\
\hline $\boldsymbol{D}_{1}$ & 22540 & 21326 & 1214 & 0.573 & 0.530 & 0.905 & 0.442 \\
$\boldsymbol{D}_{2}$ & 22562 & 21355 & 1207 & 0.426 & 0.543 & 0.775 & 0.177 \\
\hline
\end{tabular}

variance in the family parameters (model $\boldsymbol{D}_{2}$ ) lead to a more parsimonious model than the model with a different dummy rule for each family, $\boldsymbol{D}_{1}$. However, the decrease in model complexity did not compensate the value of the DIC for the increase in model misfit.

As expected, decreasing the number of second-level parameters in the model resulted in less variance explained in the item parameters according to $R_{b}^{2}$ for both models. Also, when fewer parameters were present in the model to explain the family difficulty parameters, the item difficulty parameters were less pooled around their family means, as indicated by $\lambda_{b}$. This was also reflected by the larger estimates of the within-family variance of the difficulty parameters (see Table 2.2) for the restrictive model.

Tables 2.2, 2.3, and 2.4 present the EAP estimates and the 95\% HPD intervals for the parameters for the radicals, the common covariance matrices, and the family means for the discrimination and difficulty parameters for both models. The two radicals of interest in model $\boldsymbol{D}_{2}$ had a large positive effect on the difficulty of the items (Table 2.2). Thus, both an increase of the number of transformations needed to solve the item and the number of basic elements in it reduced the probability of a correct answer. The effect of the number of basic elements was larger than the effect of the number of transformations.

In both models, the estimate of the common family covariance matrix revealed negative covariance between the discrimination and difficulty parameters. Both within (Table 2.2) and between the families (Tables 2.3 and 2.4), the easier items tended to have larger discrimination parameters than the more difficult items.

The mean discrimination per family was very similar for both models (see Table 2.3). However, the mean family difficulty showed some variation (see Table 2.4). To get an indication as to which families were especially biased by the linear approximation in model $\boldsymbol{D}_{2}$, we checked which ICM estimates of the family difficulty parameters were not included in the 95\% HPD interval of the respective LICM estimates. This comparison showed estimates for Families 1, 2, 4, and 7 that were especially biased by the linear approximation. This finding was also corroborated by the $95 \%$ HPD intervals of the differences between the empirical and modeled family means of the item difficulty parameters. For the same four families, the HPD intervals did not include zero. Based on these results, the hypothesis of two radicals in model $\boldsymbol{D}_{2}$ (the number of transformations and the number of basic elements in an item) explaining the family difficulty parameters was rejected.

Figures 2.4 (Items 1 to 15 ), 2.5 (Items 16 to 30), and 2.6 (Items 30 to 33) show the results of the Bayesian latent residual analysis for model $\boldsymbol{D}_{2}$. (The model fit 
Table 2.2: Expected a posteriori estimates and 95\% highest posterior density intervals of the radical and (co)variance parameters.

\begin{tabular}{lrr}
\hline Difficulty factors & $\boldsymbol{D}_{1}$ & $\boldsymbol{D}_{2}$ \\
\hline Intercept & - & $-3.279[-3.966,-2.623]$ \\
Number of transformations & - & $0.907[0.451,1.368]$ \\
Number of basic elements & - & $0.941[0.606,1.293]$ \\
\hline$\sigma_{a}^{2}$ & $0.062[0.018,0.118]$ & $0.081[0.024,0.155]$ \\
$\sigma_{a b}$ & $-0.012[-0.075,0.042]$ & $-0.029[-0.164,0.097]$ \\
$\sigma_{b}^{2}$ & $0.126[0.042,0.232]$ & $0.284[0.133,0.465]$ \\
\hline
\end{tabular}

Table 2.3: Expected a posteriori estimates and 95\% highest posterior density intervals of the family discrimination parameters.

\begin{tabular}{lllll}
\hline $\mathrm{f}$ & & $\boldsymbol{D}_{1}$ & & $\boldsymbol{D}_{2}$ \\
\hline 1 & 1.191 & {$[0.802,1.586]$} & 0.982 & {$[0.574,1.414]$} \\
2 & 1.226 & {$[0.860,1.629]$} & 1.188 & {$[0.824,1.574]$} \\
3 & 1.379 & {$[1.029,1.734]$} & 1.341 & {$[0.987,1.714]$} \\
4 & 0.951 & {$[0.634,1.277]$} & 1.012 & {$[0.621,1.415]$} \\
5 & 1.082 & {$[0.759,1.409]$} & 1.076 & {$[0.736,1.418]$} \\
6 & 0.728 & {$[0.409,1.035]$} & 0.764 & {$[0.402,1.136]$} \\
7 & 0.806 & {$[0.491,1.125]$} & 0.869 & {$[0.406,1.323]$} \\
8 & 0.690 & {$[0.368,1.015]$} & 0.693 & {$[0.344,1.044]$} \\
9 & 0.599 & {$[0.276,0.919]$} & 0.611 & {$[0.270,0.954]$} \\
10 & 0.665 & {$[0.316,1.020]$} & 0.689 & {$[0.283,1.104]$} \\
11 & 0.392 & {$[0.040,0.748]$} & 0.388 & {$[0.020,0.779]$} \\
\hline
\end{tabular}

Table 2.4: Expected a posteriori estimates and 95\% highest posterior density intervals of the family difficulty parameters.

\begin{tabular}{lrcrl}
\hline $\mathrm{f}$ & & $\boldsymbol{D}_{1}$ & & $\boldsymbol{D}_{2}$ \\
\hline 1 & -2.144 & {$[-2.705,-1.584]$} & -1.431 & {$[-1.737,-1.138]$} \\
2 & -1.842 & {$[-2.390,-1.337]$} & -1.431 & {$[-1.737,-1.138]$} \\
3 & -1.707 & {$[-2.172,-1.249]$} & -1.431 & {$[-1.737,-1.138]$} \\
4 & -0.911 & {$[-1.336,-0.495]$} & -1.431 & {$[-1.737,-1.138]$} \\
5 & -0.604 & {$[-1.020,-0.184]$} & -0.489 & {$[-0.839,-0.142]$} \\
6 & -0.178 & {$[-0.593,0.229]$} & -0.524 & {$[-0.980,-0.064]$} \\
7 & 0.258 & {$[-0.161,0.669]$} & -0.489 & {$[-0.839,-0.142]$} \\
8 & 0.225 & {$[-0.190,0.657]$} & 0.418 & {$[0.109,0.740]$} \\
9 & 0.536 & {$[0.117,0.973]$} & 0.418 & {$[0.109,0.740]$} \\
10 & 1.005 & {$[0.530,1.472]$} & 1.359 & {$[0.889,1.843]$} \\
11 & 0.819 & {$[0.322,1.314]$} & 0.815 & {$[0.159,1.492]$} \\
\hline
\end{tabular}


analyses for model $\boldsymbol{D}_{1}$ yielded almost the same results, due to the similarity of their first-level parameters.) For each item, the probabilities of the responses being outliers (y-axis), as computed by (2.15), were plotted against the EAP estimated ability parameters for the persons giving the responses (x-axis). Of the 20,863 responses, the probabilities of some $0.0008 \%$ were computed as greater than one. In Figure 2.4, these probabilities were set equal to one.

The outlying probabilities for the correct and the incorrect responses were plotted in the same figures. The curves can be distinguished by their form; the upward trends relate to the incorrect responses, whereas the downward trends relate to the correct responses. The former can be explained as follows. When a person of high ability gives an incorrect response to an easy item, the probability of the response being an outlier is larger than that of a person of low ability giving an incorrect response to the same item. The downward trends for the correct responses are explained analogously.

In our model fit analysis, we did not find any indication of model misfit due to guessing. If guessing had occurred, large outlying probabilities for the correct responses should have been observed. However, only five of the items yielded large probabilities for the incorrect responses (Items 1, 4, 7, 10, and 13). These items were the first items from the first five families. For most test takers, they were the first items met in the adaptive test. Therefore, it seems likely that they needed to warm up to the task - a process that might have resulted in more incorrect answers than expected given their ability level.

Note also the effect of the adaptive method of item administration in the plots. The first few items in the first series (Items 1, 4, 7, etc.) were answered by almost all test takers and, consequently, a relatively large range of estimated abilities on the x-axis was observed. The items in the second (Items 2, 5, 8, etc.) and third (Items 3, 6, 9, etc.) series were only answered by test takers with an estimated ability at the location of the difficulty of the item. For example, Item 3 (Family 1) was only answered by test takers with a negative ability estimate, whereas most of the test takers answering Item 33 (Family 11) had a positive ability estimate.

\subsubsection{Conclusion}

By comparing the fit of LICM models of different complexity, hypotheses with regard to the radicals can be tested. Based on such analyses, the set of radicals can be adapted until the selected combination of radicals satisfactorily explains the family difficulty parameters. Such a set of calibrated radicals facilitates item generation on-the-fly. For instance, in adaptive testing, based on a test takers' updated ability estimate, a family can be optimally selected (see Glas \& van der Linden, 2003), and a new item can be generated according to the design of the selected family as specified in the design matrix. Subsequently, after an answer has been given to the item, the ability estimate of the test taker can be updated using the estimates of the family distributions $\boldsymbol{\mu}_{f}$ and $\boldsymbol{\Sigma}_{f}$. 

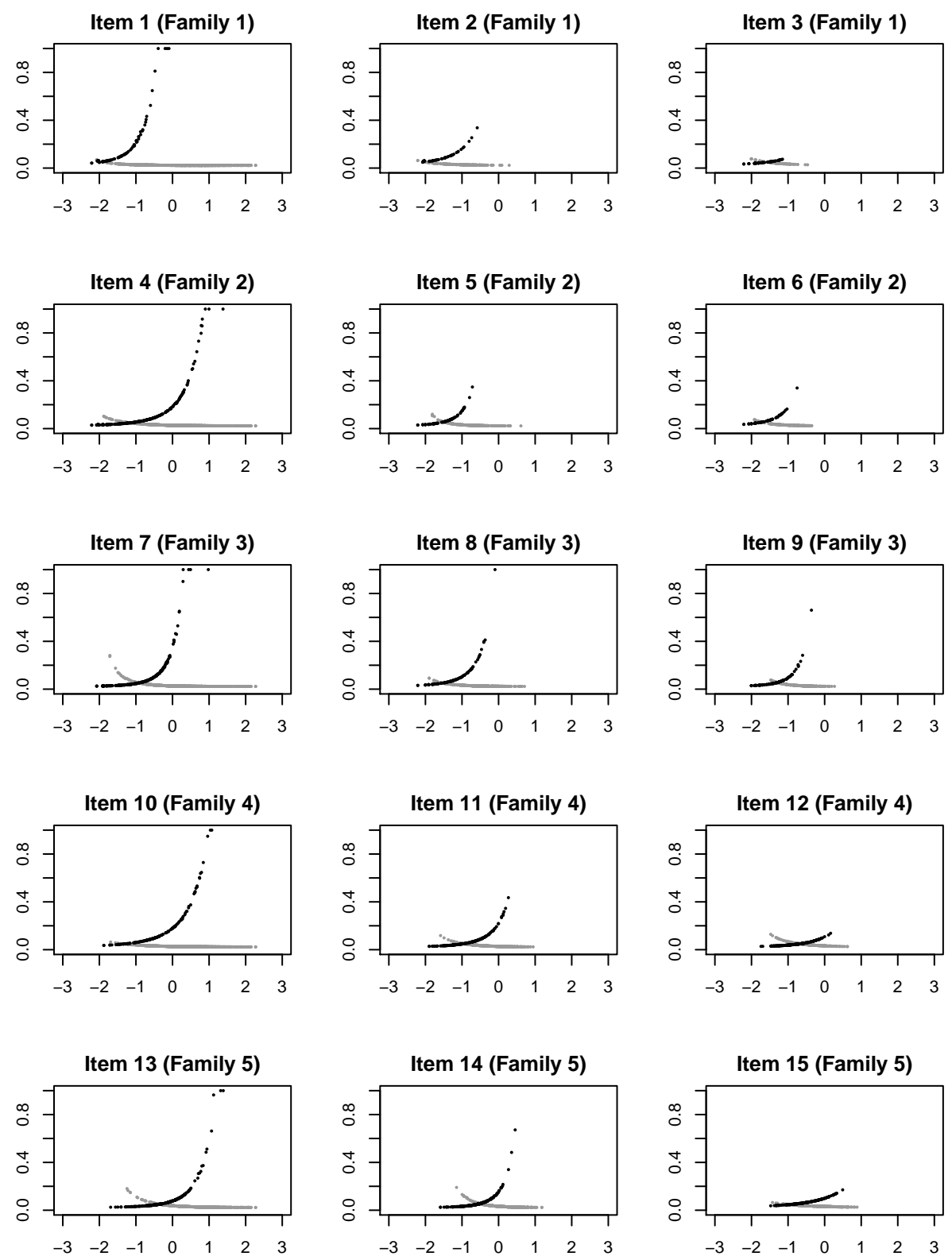

Figure 2.4: Outlying probabilities as a function of the estimated ability parameter for the items in the first five families. The upward trends relate to the incorrect responses; the downward trends relate to the correct responses. 

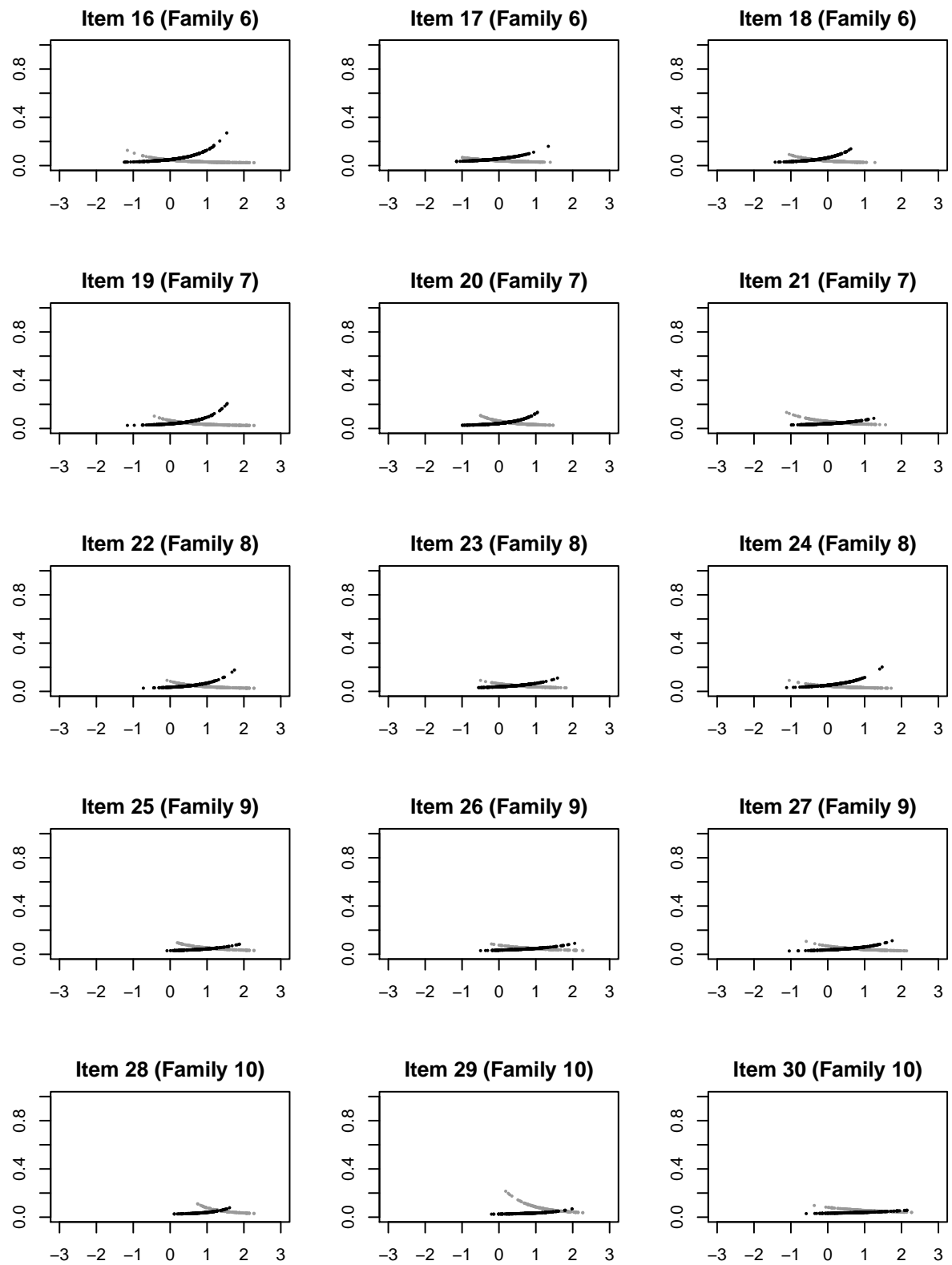

Figure 2.5: Outlying probabilities as a function of the estimated ability parameter for the items in Family 6 to 10. The upward trends relate to the incorrect responses; the downward trends relate to the correct responses. 

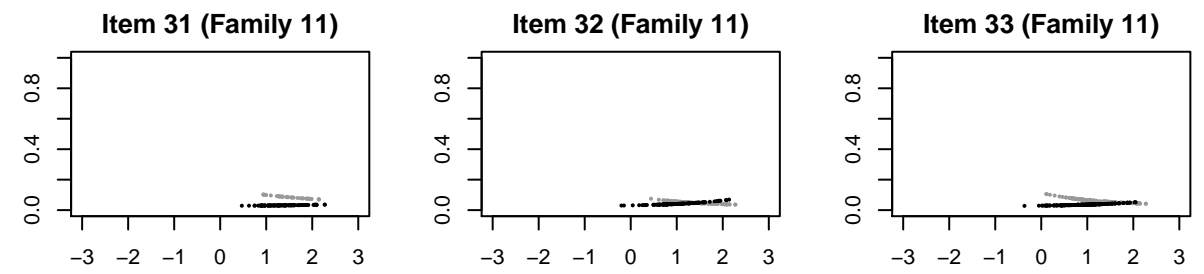

Figure 2.6: Outlying probabilities as a function of the estimated ability parameter for the items in Family 11. The upward trends relate to the incorrect responses; the downward trends relate to the correct responses.

\subsection{Simulation Study}

A parameter recovery study was conducted to explore the impact of the sampling design on the calibration of the second-level parameters in the model. In this study, each design meets the requirement that every test taker responds to one item from every family. The research question is: Conditional on the number of test takers, how should we sample the items? More specifically, if our goal is to estimate the second-level parameters as accurately as possible, would it be better to sample:

- more items per family, reducing the numbers of test takers per item, or

- fewer items per family, thereby increasing the numbers of test takers per item?

\subsubsection{Study Setup}

The design of this study consisted of runs with 8 or 16 families and 10 or 20 items per family. The total number of test takers was fixed at either 500 or 1000 . Each cell of the design was replicated 20 times. The data were simulated and the parameters were estimated under the assumption of equal covariance matrices across families (LICM-C) with the $2 \mathrm{PNO}$ as the first-level model. The choice for the $2 \mathrm{PNO}$ was made for practical reasons only. In Appendix A.2 it is explained that the conditional posterior distribution of the guessing parameters is not a standard distribution, and therefore a method such as importance sampling would have been needed to sample for these parameters. Consequently, more iterations would have been required for the Gibbs sampler to converge. Because of the large number of runs in our study, the total running time would have been prohibitive, while the feasibility of the method for sampling $\gamma_{i_{f}}$ has already been demonstrated by Glas et al. (2010).

As the simulated test takers were required to respond to one item from every family, the number of observations per item $N_{i_{f}}$ was equal to the total number of test takers $N_{f}$ divided by the number of items per family $I_{f}$. Parameters $\boldsymbol{\beta}$ were set equal to $2.9,1.1,0.8$, so that the application of a rule had a positive effect on the difficulty of the items; the common effect was chosen to be -2.7 . 
For the case of eight families, a full factorial design was used:

$$
\boldsymbol{D}=\left[\begin{array}{llll}
1 & 0 & 0 & 0 \\
1 & 0 & 0 & 1 \\
1 & 0 & 1 & 0 \\
1 & 0 & 1 & 1 \\
1 & 1 & 0 & 0 \\
1 & 1 & 0 & 1 \\
1 & 1 & 1 & 0 \\
1 & 1 & 1 & 1
\end{array}\right]
$$

For the case of 16 families, the rows in the matrix were duplicated. This was done to avoid confounding of the number of families with the structure of the design matrix when comparing the precision of parameter estimates. Without duplication of the design matrix, adding new families would have required the introduction of more radicals, and would have resulted in a design matrix with a different structure for the conditions with 16 families than the design matrix for the conditions with eight families. Note that, in the estimation procedure, families with the same combination of radicals will have the same estimated family difficulty parameter, but their estimated discrimination parameters are allowed to vary.

The family discrimination parameters were fixed at one and the difficulty parameters at $\boldsymbol{D} \boldsymbol{\beta}$. The covariance matrices were chosen to be equal across families (LICM-C model); the diagonal of the common matrix was equal to $(.10, .10)$ and the off-diagonal elements were equal to .05. In every replication, the item parameters were randomly drawn from their family distributions, according to (2.4). Negative draws for the discrimination parameters were discarded. The ability parameters were drawn from the standard normal distribution.

The prior means of the family discrimination and effect parameters $\boldsymbol{\lambda}_{0}$ as well as the prior scale matrix $\boldsymbol{S}_{0}$ were set equal to their true values. Furthermore, $\boldsymbol{V}_{0}$ was set equal to the $F+R$ identity matrix and $\nu_{0}$ was set equal to two (i.e., smallest value of $\nu_{0}$ given the dimension of the covariance matrix; Gelman et al., 2004).

The convergence diagnostics of Geweke (1992), Heidelberger and Welch (1983), and Raftery and Lewis (1992) were used to determine the total number of iterations as well as the number of burn-in iterations needed for the Gibbs sampler to reach satisfactory convergence. To reduce the total running time for the study, we used the true values of the parameters as starting values to create a smaller number of burn-in iterations - a choice that did not influence the final results. In every replication, 32,000 iterations were performed, 2,000 of which had to be discarded as a burn-in. Following a recommendation by MacEachern and Berliner (1994), the Gibbs sampler was not subsampled and all iterations after burn-in were used to compute both the EAP estimates of the parameters as well as their mean absolute error (MAE), bias, and variance.

\subsubsection{Results}

The first three columns of Table 2.5 give the number of families $F$, items per family $I_{f}$, and persons $N_{f}$ in the design, respectively. Also, the resulting number 
of observations per item $N_{i_{f}}$ is given. The next three columns give the MAEs for the first-level parameter estimates, and the last columns give the MAEs for the second-level parameter estimates.

Because the values for the MAE depend on the scale that was set for the model, the content of the table should be interpreted in a relative manner; that is, to compare between the conditions. However, to give some feeling for the size of the MAEs, the results can be compared to the results for the two-parameter logistic (2PL) model reported in van der Linden and Glas (2000). These authors also fixed the scale by setting $\boldsymbol{\theta} \sim N(0,1)$. With a test length of 20 items and a true ability value of zero, they found a MAE for the estimated ability parameters of approximately 0.3 when using a weighted maximum likelihood estimation procedure and a maximum-information criterion for selecting the items from an item pool consisting of 400 items (van der Linden \& Glas, 2000, Figure 5). In our simulation study, we found a similar value of approximately 0.35 with a test length of 16 items and an item pool of 320 items (Table 2.5; sixth row).

In general, the higher-level parameters were better recovered than the firstlevel parameters. Because every simulated test taker responded to one item from every family, the conditions with 16 families resulted in a lower MAE for the ability parameters and, consequently, in lower MAEs for all other parameters, as compared to the conditions with only eight families.

In addition, the number of families had a larger effect on the MAE of the estimated family difficulty parameters than for the estimated family discrimination parameters. This was as expected. Across families, every item with a common effect parameter $\beta_{r}$ contributes to the accuracy of the estimate of this parameter and, consequently, to the accuracy of the family difficulty estimates computed from the estimate. In contrast, only items in the same family contribute to the estimate of its discrimination parameter.

Generally, the MAEs for the covariance matrix were small. This finding is assumed to be due to the assumption of a common matrix across families: Under the assumption, the estimate of the covariance matrix is based on $F * I_{f}$ observationsa product ranging from 80 to 320 in this study. When family-specific covariance matrices are to be estimated (i.e., when the LICM-F is used), only $I_{f}$ observations are available to estimate each covariance matrix.

To answer our earlier research question, for each level $F$, the rows of the table with equal $N_{f}$ should be compared. Generally, a larger number of observations per item resulted in lower MAEs for the item parameters. However, for the secondlevel parameters, lower MAEs were obtained for larger numbers of items per family, even though this resulted in lower numbers of observations per item.

Tables 2.6 and 2.7 present the results in terms of mean bias and mean variance. In a simulation study with a limited number of replications, it is important to check whether the results could be due to chance. Therefore, for all types of parameters, $t$-tests for independent samples were conducted on the mean bias and the mean variances to check whether their differences between conditions with an equal number of families $F$ and an equal number of test takers $N_{f}$ were significant over the 20 replications. At a level of 0.05 , the $t$-tests on the mean bias did not reveal any significant differences between the conditions. In all conditions, the bias was small, and differences between the conditions should be due to chance. However, 
Table 2.5: Mean absolute error of the estimated first-level and second-level parameters as a function of the number of families $F$, items within families $I_{f}$ and persons $N_{f}$.

\begin{tabular}{ccllccccccc}
\hline$F$ & $I_{f}$ & $N_{f}$ & $N_{i_{f}}$ & $\boldsymbol{\theta}$ & $\boldsymbol{a}$ & $\boldsymbol{b}$ & $\boldsymbol{\mu}_{a}$ & $\boldsymbol{\mu}_{b}$ & $\boldsymbol{\beta}$ & $\boldsymbol{\Sigma}$ \\
\hline 8 & 10 & 500 & 50 & 0.445 & 0.215 & 0.205 & 0.145 & 0.114 & 0.119 & 0.027 \\
& 20 & 500 & 25 & 0.455 & 0.241 & 0.220 & 0.129 & 0.103 & 0.118 & 0.023 \\
\hline & 10 & 1000 & 100 & 0.444 & 0.196 & 0.170 & 0.116 & 0.091 & 0.102 & 0.020 \\
& 20 & 1000 & 50 & 0.443 & 0.214 & 0.194 & 0.112 & 0.083 & 0.086 & 0.018 \\
\hline \multirow{2}{*}{16} & 10 & 500 & 50 & 0.352 & 0.205 & 0.188 & 0.122 & 0.065 & 0.056 & 0.017 \\
& 20 & 500 & 25 & 0.347 & 0.226 & 0.207 & 0.108 & 0.063 & 0.068 & 0.018 \\
\hline & 10 & 1000 & 100 & 0.343 & 0.173 & 0.159 & 0.097 & 0.052 & 0.055 & 0.019 \\
& 20 & 1000 & 50 & 0.341 & 0.200 & 0.183 & 0.079 & 0.056 & 0.044 & 0.017 \\
\hline
\end{tabular}

Table 2.6: Mean bias of the estimated first-level and second-level parameters as a function of the number of families $F$, items within families $I_{f}$ and persons $N_{f}$.

\begin{tabular}{rrlrrrrrrr}
\hline$F$ & $I_{f}$ & $N_{f}$ & $\boldsymbol{\theta}$ & $\boldsymbol{a}$ & $\boldsymbol{b}$ & $\boldsymbol{\mu}_{a}$ & $\boldsymbol{\mu}_{b}$ & $\boldsymbol{\beta}$ & $\boldsymbol{\Sigma}$ \\
\hline 8 & 10 & 500 & 0.012 & 0.029 & 0.001 & 0.034 & 0.001 & 0.015 & 0.001 \\
& 20 & 500 & 0.015 & 0.039 & 0.020 & 0.038 & 0.019 & 0.026 & -0.001 \\
\hline & 10 & 1000 & -0.006 & 0.021 & -0.010 & 0.018 & -0.015 & 0.005 & 0.001 \\
& 20 & 1000 & 0.002 & 0.012 & 0.009 & 0.019 & 0.008 & 0.010 & 0.003 \\
\hline \multirow{2}{*}{16} & 10 & 500 & -0.014 & 0.033 & -0.004 & 0.027 & 0.003 & 0.010 & 0.003 \\
& 20 & 500 & 0.004 & 0.019 & -0.008 & 0.016 & -0.013 & -0.001 & 0.000 \\
\hline & 10 & 1000 & -0.015 & 0.014 & -0.024 & 0.025 & -0.009 & 0.002 & 0.002 \\
& 20 & 1000 & -0.016 & -0.002 & -0.018 & 0.003 & -0.010 & -0.005 & -0.003 \\
\hline
\end{tabular}

Table 2.7: Mean variance of the estimated first-level and second-level parameters as a function of the number of families $F$, items within families $I_{f}$ and persons $N_{f}$.

\begin{tabular}{cccccccccc}
\hline$F$ & $I_{f}$ & $N_{f}$ & $\boldsymbol{\theta}$ & $\boldsymbol{a}$ & $\boldsymbol{b}$ & $\boldsymbol{\mu}_{a}$ & $\boldsymbol{\mu}_{b}$ & $\boldsymbol{\beta}$ & $\boldsymbol{\Sigma}$ \\
\hline 8 & 10 & 500 & 0.304 & 0.074 & $\mathbf{0 . 0 6 8}$ & $\mathbf{0 . 0 3 5}$ & $\mathbf{0 . 0 2 0}$ & $\mathbf{0 . 0 2 1}$ & 0.001 \\
& 20 & 500 & 0.310 & 0.081 & $\mathbf{0 . 0 8 0}$ & $\mathbf{0 . 0 2 8}$ & $\mathbf{0 . 0 1 6}$ & $\mathbf{0 . 0 1 7}$ & 0.001 \\
\hline & 10 & 1000 & 0.305 & 0.057 & $\mathbf{0 . 0 4 5}$ & $\mathbf{0 . 0 2 4}$ & $\mathbf{0 . 0 1 3}$ & $\mathbf{0 . 0 1 4}$ & $\mathbf{0 . 0 0 1}$ \\
& 20 & 1000 & 0.308 & 0.063 & $\mathbf{0 . 0 5 9}$ & $\mathbf{0 . 0 1 7}$ & $\mathbf{0 . 0 1 0}$ & $\mathbf{0 . 0 1 1}$ & $\mathbf{0 . 0 0 1}$ \\
\hline 16 & 10 & 500 & 0.179 & $\mathbf{0 . 0 6 8}$ & $\mathbf{0 . 0 5 8}$ & $\mathbf{0 . 0 2 7}$ & $\mathbf{0 . 0 1 0}$ & $\mathbf{0 . 0 0 9}$ & $\mathbf{0 . 0 0 1}$ \\
& 20 & 500 & 0.185 & $\mathbf{0 . 0 7 5}$ & $\mathbf{0 . 0 6 8}$ & $\mathbf{0 . 0 2 1}$ & $\mathbf{0 . 0 0 8}$ & $\mathbf{0 . 0 0 7}$ & $\mathbf{0 . 0 0 1}$ \\
\hline & 10 & 1000 & $\mathbf{0 . 1 7 7}$ & $\mathbf{0 . 0 4 7}$ & $\mathbf{0 . 0 3 9}$ & $\mathbf{0 . 0 1 9}$ & $\mathbf{0 . 0 0 6}$ & $\mathbf{0 . 0 0 6}$ & $\mathbf{0 . 0 0 0}$ \\
& 20 & 1000 & $\mathbf{0 . 1 8 5}$ & $\mathbf{0 . 0 5 7}$ & $\mathbf{0 . 0 5 2}$ & $\mathbf{0 . 0 1 3}$ & $\mathbf{0 . 0 0 5}$ & $\mathbf{0 . 0 0 5}$ & $\mathbf{0 . 0 0 0}$ \\
\hline
\end{tabular}

The boldfaced numbers indicate a significant difference between conditions with an equal number of families $F$ and an equal number of test takers $N_{f}$ at the 0.05 level. 
the $t$-tests on the mean variances did produce significant results (see Table 2.7). In all comparisons, the family mean parameters $\boldsymbol{\mu}_{a}$ and $\boldsymbol{\mu}_{b}$, and the effect parameters $\boldsymbol{\beta}$ were estimated with higher precision when the number of items within families was larger.

\subsubsection{Conclusion}

A conspicuous advantage of the ICM and the LICM is that they do not require the calibration of newly generated items from families. However, the advantage does require an investment in the form of the calibration of the families (i.e., estimation of the second-level parameters). As shown in the simulation study, careful balancing between the number of items per family and the number of persons to whom an item from it is administered reduces the amount of data to be collected to obtain a certain precision.

\subsection{Discussion}

A model for the calibration of items generated by rule-based cloning algorithms was presented. The model is applicable to situations where items within families are generated by the same rules. These rules ("radicals") are modeled as fixed effects whereas the joint effects of all irrelevant item features ("incidentals") are modeled as random effects. In the current testing practice, it is still somewhat unusual to consider item parameters as random. But from a theoretical viewpoint it makes sense to view them as sampled from families defined by different content rules. For a more complete discussion of the advantages of treating item parameters as random, see De Boeck (2008).

In practical applications, the status of the radicals and incidentals as fixed and random effects should be checked against empirical results. For example, for the earlier example of a statistics item, one might expect the actual numerical information provided in the context story not to influence the difficulty of the item. However, the assumption has to be rejected when some types of numerical information, for example large numbers, lead to substantially higher difficulty estimates than others. In such cases, an assumed incidental should be turned into a radical.

Of course, the success of automated item generation depends on how well the radicals explain the family difficulty parameters. For instance, an assumed effect of a radical may not be found. Also, the possibility of interaction effects between radicals should be considered. Procedures have been presented to investigate the degree of misfit due to unexplained variance in the family difficulty parameters. If the degree of misfit is unacceptable and the cause for misfit can be identified, it may be worth considering an adaptation of the model. Alternatively, it would be interesting to investigate whether the model can be extended with an error term to account for residual variance in the family difficulty parameters.

Also, a basic assumption of the model is that the radicals are assumed to have the same effect for every individual test taker, but this may not always hold. For example, if different strategies for an item lead to the same solution, a radical may 
be more difficult for a student using one strategy but easier for a student using another. Other models may then provide a better description of the data.

As for the choice of incidentals, a trade-off exists between the likelihood of generating items with a different appearance and keeping the family covariance matrices small. Smaller covariance matrices are expected to lead to more accurate estimation of the ability parameters. Also, the amount of uncertainty in the item parameters influences the information provided by a test assembled from a pool of item families using optimal test assembly methods. 



\title{
Chapter 3
}

\section{Testing Construction Rules for Intelligence Tests}

\begin{abstract}
Item response theory models with a structural model on the item parameters can be used to test hypotheses regarding the effect of item features on item difficulty and to predict the difficulty of newly constructed test items. A comparison is made between two such models: one that assumes a perfect prediction and another that allows for residual variance in the item parameters. The models are used to test the difficulty theory of two subtests of the SON-R $51 / 2-17$, a nonverbal test of intelligence. The effect of the degree of residual variance in the item parameters on the robustness of the results is investigated through a simulation study.
\end{abstract}

Key words: difficulty theory, item response theory, linear logistic test model, model misspecification.

\subsection{Introduction}

After the development of the linear logistic test model (LLTM) by Fischer (1973) a large number of studies have been conducted to investigate the effect of item construction rules on the difficulty of intelligence and achievement test items. The LLTM has been applied, for example, to figural matrices (Hornke \& Habon, 1986; Freund et al., 2008; Kubinger, 2008), word problems (Holling, Blank, Kuchenbäcker, \& Kuhn, 2008; Holling et al., 2009), and reading comprehension items (Gorin, 2005; Gorin \& Embretson, 2006; Sonnleitner, 2008). Apart from testing hypotheses regarding the factors influencing the difficulty of items, the LLTM can also be used to estimate the ability of test takers based on predictions regarding the difficulty of newly constructed test items. However, due to its assumptions of equal discrimination parameters and a perfect prediction of the difficulty parameters by

Adapted from: Geerlings, H., Laros, J. A., Tellegen, P. J., \& Glas, C. A. W. (2011). Testing the difficulty theory of the SON-R 51/2-17, a nonverbal test of intelligence. Submitted for publication. 
the item construction rules, the LLTM is a relatively restrictive model.

The restriction of equal discrimination parameters is often made for computational reasons; the existence of a sufficient statistic for the ability parameter allows for conditional maximum likelihood estimation (Andersen, 1972), which has the advantage that it does not involve assumptions regarding a distribution for ability parameters. If the restriction of equal discrimination parameters is violated, misfitting items are either removed from the item pool, or the model is adapted to allow for item-specific discriminatory power. In the latter case, the increase in model complexity requires a corresponding increase in the size of the calibration sample. As alternatives, the discrimination parameters can be substituted as known constants (Verhelst \& Glas, 1995), or, in a Bayesian context, they can be given a hierarchical prior so that they can borrow strength through their common mean (Geerlings, Glas, \& van der Linden, 2011; Chapter 2).

As mentioned above, the restriction that the item difficulty parameters equal a sum of item feature effects has the advantages of allowing for hypothesis testing and prediction. However, the prediction of item difficulty parameters based on item features is not perfect, and ignoring the residual variance in the difficulty parameters results in underestimates of the standard errors associated with the feature effect parameters (De Boeck, 2008). As an alternative, an error term can be added to the model to capture the residual variance (Janssen et al., 2004).

In this article, a model is suggested that allows both discrimination and difficulty parameters to deviate from their mean values as imposed by a common discrimination parameter and a feature model for item difficulty, respectively. The model is a restricted version of the linear item cloning model of Geerlings et al. (2011; Chapter 2), a hierarchical model for rule-based generated items. In the next section, a normal ogive equivalent of the LLTM and the extended model will be discussed in more detail. An empirical study in which both models are applied to two of the subtests of the SON-R 51/2-17, a nonverbal test of intelligence (Laros \& Tellegen, 1991; Tellegen \& Laros, 1993), will be described in Section 3.3. In Section 3.4, a simulation study will be presented in which the effect of model choice on the estimates of the feature effect and ability parameters is investigated. The article will end with a discussion on the consequences of the results for the two model purposes of hypothesis testing and ability estimation based on a prediction of item difficulty.

\subsection{Modeling Approach}

The LLTM is an example of a so called explanatory item response theory (IRT) model (De Boeck \& Wilson, 2004). In IRT, the effects of persons and items on the responses are modeled by different sets of parameters. The respective person and item parameters are estimated on the same scale, which enables the comparison of scores even when different persons have been administered different items. By extending IRT models with structural models on the item parameters, the models serve not only measurement purposes but also the explanatory purposes discussed in the introduction. In the next sections, the specific models used in the studies below, a Bayesian parameter estimation procedure and model fit measures will be discussed. 


\subsubsection{Response Models}

Let $U_{i n}$ be a response variable indicating whether person $n, n=1, \ldots, N$, completed item $i, i=1, \ldots, K$, correctly $\left(U_{i n}=1\right)$ or incorrectly $\left(U_{i n}=0\right)$, and let $\theta_{n}$ be this person's ability parameter. Furthermore, $\boldsymbol{\xi}_{i}=\left(a_{i}, b_{i}\right)$ are the item parameters. $b_{i}$ is a location parameter, and indicates the difficulty of item $i . a_{i}$ is a slope parameter, and indicates the degree to which the item discriminates between abilities located near this difficulty level. The probability of a correct response can be given by either

$$
P\left(U_{i n}=1 \mid \theta_{n}, \boldsymbol{\xi}_{i}\right)=\Psi\left(a_{i}\left[\theta_{n}-b_{i}\right]\right),
$$

or

$$
P\left(U_{i n}=1 \mid \theta_{n}, \boldsymbol{\xi}_{i}\right)=\Phi\left(a_{i}\left[\theta_{n}-b_{i}\right]\right),
$$

where $\Psi($.$) is the logistic function, and \Phi($.$) is the cumulative normal distribu-$ tion function. The models are known as the two-parameter logistic (2PL) and two-parameter normal ogive (2PNO) model, respectively, and if the argument of $\Psi($.$) is multiplied by a scaling factor of 1.7$, the resulting probabilities are approximately the same. Note that the discrimination parameter weights the item-person difference. If $a_{i}$ equals one, the probability of a correct response equals 0.5 if a person's ability $\theta_{n}$ equals the difficulty $b_{i}$ of the item. Three basic assumptions of the models are that a unidimensional trait accounts for the ability of the persons, that the probability of a correct response increases with ability, and that the responses of a person are independent given the model parameters.

Fischer's (1973) LLTM is obtained by adding two restrictions to (3.1). First, it is assumed that all discrimination parameters are equal. Second, it is assumed that the difficulties of the items can be explained by a linear combination of effect parameters. In this article, we will use the normal ogive version of the model, parameterized as $\Phi\left(a_{i} \theta_{n}-b_{i}\right)$, because estimates are made in a Bayesian framework, and this representation allows for easy sampling from the conditional posterior distributions of the parameters through data augmentation (for details, see Section 3.2.2 and Appendix A). In this representation, the discrimination parameter can be interpreted as the slope, and the difficulty parameter as the intercept in a generalized nonlinear model. The model, which will be labeled the linear one-parameter normal ogive (L1PNO), adds the following restrictions to the item parameters:

$$
\begin{aligned}
a_{i} & =\mu_{a}, \\
b_{i} & =\sum_{r=1}^{R} d_{i r} \beta_{r},
\end{aligned}
$$

where $\mu_{a}$ is the mean value for the discrimination parameters, $\beta_{r}$ is the effect of item feature $r$ on the difficulty of the items and $d_{i r}$ is a design variable denoting how many instantiations of feature $r$ are present in item $i$. The difficulty of an item is thus equal to a weighted sum of the item feature effects.

To account for unequal discrimination parameters and residual variance in the item difficulty parameters, the model can be adapted by modeling the item parameters as random variables. This leads to a multilevel model in which the first level equals the reparameterized $2 \mathrm{PNO}$ model and the second level consists 
of multivariate normal distributions for the item parameters,

$$
\boldsymbol{\xi}_{i} \sim M V N\left(\boldsymbol{\mu}_{i}, \boldsymbol{\Sigma}\right)
$$

with $\boldsymbol{\mu}_{i}=\left(\mu_{a_{i}}, \mu_{b_{i}}\right)$ the mean values for item $i$ imposed by the linear structures described below, and $\operatorname{Vec}(\boldsymbol{\Sigma})=\left(\sigma_{a}^{2}, \sigma_{a b}, \sigma_{b}^{2}\right)$ a covariance matrix denoting the variability of the item parameters around their mean values. The mean parameters are restricted in a manner analogous to (3.3) and (3.4):

$$
\begin{aligned}
\mu_{a_{i}} & =\mu_{a}, \\
\mu_{b_{i}} & =\sum_{r=1}^{R} d_{i r} \beta_{r} .
\end{aligned}
$$

The model, which will be labeled the L1PNO-C, is a restricted version of the linear item cloning model with a common covariance matrix (LICM-C) of Geerlings et al. (2011; Chapter 2), a model developed for sets of items, 'families', with family membership determined by the combination of rules used to generate the items. The L1PNO-C is the special case in which each item is considered a oneitem family, and all families have the same mean discrimination parameter $\mu_{a}$. Furthermore, as the LICM-C was presented including guessing parameters, these parameters should be set to zero for the L1PNO-C.

\subsubsection{Parameter Estimation}

In the studies described below, a Bayesian method (Gibbs sampling; see for example Casella \& George, 1992) was used to estimate the parameters of the models. In Bayesian analyses, inferences are based on the posterior distribution of the parameters. Let $\boldsymbol{\beta}=\left(\beta_{r}\right)$ be the feature effect parameters and $\boldsymbol{D}=\left(\left(d_{i r}\right)\right)$ the design matrix. The other boldfaced parameters are defined analogously. The joint posterior of the L1PNO-C is

$$
p(\boldsymbol{z}, \boldsymbol{\theta}, \boldsymbol{\xi}, \boldsymbol{\lambda}, \boldsymbol{\Sigma} \mid \boldsymbol{u}, \boldsymbol{D}) \propto p(\boldsymbol{z} \mid \boldsymbol{u}, \boldsymbol{\theta}, \boldsymbol{\xi}) p(\boldsymbol{\xi} \mid \boldsymbol{D}, \boldsymbol{\lambda}, \boldsymbol{\Sigma}) p(\boldsymbol{\lambda}) p\left(\boldsymbol{\Sigma} \mid \boldsymbol{S}_{0}, \nu_{0}\right) p(\boldsymbol{\theta}),
$$

where $\boldsymbol{\lambda}=\left(\mu_{a}, \boldsymbol{\beta}\right)$ are the regression parameters for the means. The variables $\boldsymbol{Z}=$ $\left(\left(Z_{i n}\right)\right)$ are data augmentation variables, which simplify the estimation algorithms described in Appendix A. Furthermore, $\boldsymbol{S}_{0}$ and $\nu_{0}$ are parameters for the prior density of $\boldsymbol{\Sigma}$. The joint posterior of the L1PNO is obtained from (3.8) by restricting $\boldsymbol{\Sigma}$ to be zero, corresponding to the assumption of no residual variance, so that $p(\boldsymbol{\xi} \mid \boldsymbol{D}, \boldsymbol{\lambda}, \boldsymbol{\Sigma})$ evaluates to one. That is,

$$
p(\boldsymbol{z}, \boldsymbol{\theta}, \boldsymbol{\lambda} \mid \boldsymbol{u}, \boldsymbol{D}) \propto p(\boldsymbol{z} \mid \boldsymbol{u}, \boldsymbol{\theta}, \boldsymbol{D}, \boldsymbol{\lambda}) p(\boldsymbol{\lambda}) p(\boldsymbol{\theta}) .
$$

In the studies described below, we will use the prior $p(\boldsymbol{\theta}) \sim N(0,1)$. So its mean and variance serve as an identification restriction. The following noninformative prior was used for $\boldsymbol{\lambda}$ in both models:

$$
p(\boldsymbol{\lambda})=I\left(\mu_{a}>0\right) ;
$$


that is, the mean discrimination parameter was restricted to be positive. The prior for the covariance matrix in the L1PNO-C was the inverse-Wishart distribution with sum of squares $\boldsymbol{S}_{0}$ and $\nu_{0}$ degrees of freedom,

$$
\boldsymbol{\Sigma} \sim \text { inverse-Wishart }\left(\boldsymbol{S}_{0}, \nu_{0}\right)
$$

Expected a posteriori (EAP) estimates of the model parameters can be computed from draws taken iteratively from the conditional distributions of subsets of the parameters given all others. These conditional distributions often have standard forms that are easily sampled from. The specific Gibbs sampling algorithms used to estimate the parameters of the L1PNO and L1PNO-C model are based on an algorithm for the $2 \mathrm{PNO}$ constructed by Albert (1992) and are described in detail in Appendix A. Convergence of the draws to the full posterior distribution can be assessed using for example diagnostics by Geweke (1992) and Heidelberger and Welch (1983).

After calibration, the EAP estimates $\hat{\boldsymbol{\lambda}}$ (L1PNO) or $\hat{\boldsymbol{\lambda}}$ and $\hat{\boldsymbol{\Sigma}}$ (L1PNO-C) can be used to estimate the ability of new test takers based on their responses to new (uncalibrated) items that have been generated using the calibrated item construction rules. Let $P_{i}\left(\theta_{n}\right)$ denote the probability of a correct response of test taker $n$ on item $i$; that is,

$$
\begin{array}{rlr}
P_{i}\left(\theta_{n}\right) & =P\left(U_{i n}=1 \mid \theta_{n}, \boldsymbol{D}_{i}, \hat{\boldsymbol{\lambda}}\right), & \\
P_{i}\left(\theta_{n}\right) & =\int P\left(U_{i n}=1 \mid \theta_{n}, \boldsymbol{\xi}_{i}\right) p\left(\boldsymbol{\xi}_{i} \mid \boldsymbol{D}_{i}, \hat{\boldsymbol{\lambda}}, \hat{\boldsymbol{\Sigma}}\right) d \boldsymbol{\xi}_{i} . &
\end{array}
$$

The latter is known as the expected response function for item $i$ and can be approximated using Monte Carlo integration (Lewis, 2001). The posterior distribution of $\theta_{n}$ given response vector $\boldsymbol{u}_{n}=\left(u_{i n}\right)$ is

$$
p\left(\theta_{n} \mid \boldsymbol{u}_{n}, \hat{\boldsymbol{\lambda}}, \hat{\boldsymbol{\Sigma}}\right)=\frac{\int \theta_{n} p(\theta) \prod_{i=1}^{K} P_{i}\left(\theta_{n}\right)^{u_{i n}}\left[1-P_{i}\left(\theta_{n}\right)\right]^{1-u_{i n}} d \theta}{\int p(\theta) \prod_{i=1}^{K} P_{i}\left(\theta_{n}\right)^{u_{i n}}\left[1-P_{i}\left(\theta_{n}\right)\right]^{1-u_{i n}} d \theta} ;
$$

see, for example, Baker and Kim (2004, section 7.5.2). Gauss-Hermite quadrature can be used to approximate the normal distribution $p(\theta)$ and to obtain the EAP estimate for $\theta_{n}$.

\subsubsection{Model Fit Assessment}

Two types of tests are presented to investigate the fit of the two models to the data. First, the assumptions of equal discrimination parameters and no residual variance in the difficulty parameters can be tested by comparisons of the item parameters $\boldsymbol{a}$ and $\boldsymbol{b}$ of the L1PNO-C with their respective means, $\boldsymbol{\mu}_{a}$ and $\boldsymbol{\mu}_{b}$. Let $\zeta$ be the parameter under investigation, and $\mu_{\zeta}$ its mean. The probability corresponding to $\zeta \geq \mu_{\zeta}$ can be computed from their Gibbs samples by counting the number of times that the inequality holds, and dividing by the total number of iterations. If $\zeta$ equals $\mu_{\zeta}$, the probability will be approximately 0.5 . Either a very small or a very large probability value is an indication that $\zeta$ is not well explained by $\mu_{\zeta}$. 
As a second type of test, posterior predictive checks (PPCs) can be performed (Gelman et al., 1996). PPCs are based on the idea that if the model fits well, data replicated under the model should look similar to the observed data. For example, a posterior predictive p-value (PPP) to assess item fit can be given as

$$
\operatorname{PPP}=P\left\{T\left(\boldsymbol{u}_{i}^{\text {rep }}, \boldsymbol{\xi}_{i}, \boldsymbol{\theta}\right) \geq T\left(\boldsymbol{u}_{i}, \boldsymbol{\xi}_{i}, \boldsymbol{\theta}\right) \mid \boldsymbol{u}\right\},
$$

where $T\left(\boldsymbol{u}_{i}^{\text {rep }}, \boldsymbol{\xi}_{i}, \boldsymbol{\theta}\right)$ is the replicated value for a test statistic, and $T\left(\boldsymbol{u}_{i}, \boldsymbol{\xi}_{i}, \boldsymbol{\theta}\right)$ is the observed value for the test statistic. Fit statistics can be defined to target the assumptions of the model under investigation and may depend either on the data only, or both on the data and the model parameters. Replicated datasets can be obtained using Gibbs samples of the model parameters. In case of missing data in the observed dataset, the missing values can simply be copied into the replicated datasets (in case of missing data due to an incomplete data-collection design) or the item-selection algorithm used in collecting the observed data can be applied to the replicated datasets to determine the missing values (in case of missing data due to adaptive testing). The PPP in (3.15) can be computed by counting across Gibbs sampler iterations the number of times that the inequality holds, and dividing by the total number of iterations. Either a very small or a very large probability value is an indication that the predicted distribution of the test statistic differs from its observed value (in case of a test statistic depending on the data only) or the observed distribution (in case of a test statistic depending on both data and model parameters).

As item fit statistics for adaptively collected data, the number of correct, incorrect, and missing responses per item can be used. For data collected according to a fixed design, the latter two statistics are superfluous. Let $y_{\text {in }}$ be a missing data indicator which equals one if the response of person $n$ to item $i$ is missing, and zero otherwise. For item $i$, the three statistics are given by

$$
T_{i}^{+}=\sum_{n=1}^{N} u_{i n}, \quad T_{i}^{-}=\sum_{n=1}^{N}\left(1-u_{i n}\right), \quad \text { and } \quad T_{i}^{N A}=\sum_{n=1}^{N} y_{i n} .
$$

The statistics are indicative of the difficulty of the item, and its observed values will therefore be well predicted by a model with individual item difficulty parameters (Sinharay, Johnson, \& Stern, 2006). The reason is that, for every item, the estimated proportion of correct item responses is only function of one difficulty parameter and the estimation procedure tends to match the observed and expected proportion correct item responses. However, in case of a linear restriction on the difficulty parameters, the statistics are useful in detecting inaccuracy of the prediction for the individual items. This is because the linear restrictions on the item parameters impose restrictions on the expected proportions correct, and as a result, the observed and expected proportions are much harder to match for every item. Therefore, the extent of this discrepancy is a measure of misfit.

\subsection{Empirical Study}

For the empirical study the dataset resulting from the standardization research of the SON-R 51/2-17 (Laros \& Tellegen, 1991; Tellegen \& Laros, 1993) was used. 
The SON-R 51/2-17 is an intelligence test for children aged between 51/2 and 17 years which can be administered individually without the use of spoken language. Therefore, the test can give a fair comparison of deaf and hearing children, and of children who do not speak the language of the test administrator. The SON-R 51/2-17 consists of seven subtests: two abstract reasoning tests (Categories and Analogies), two concrete reasoning tests (Situations and Stories), two spatial tests (Mosaics and Patterns), and one perceptual test (Hidden Pictures). Except for the last test, all tests were constructed according to a theory of difficulty. This enabled the authors to create items within several levels of difficulty.

The items were divided into two or three series of items, depending on the subtest, in such a way that every series contains a range of items from very easy to very hard. The different series of items are used in an adaptive administration procedure of the test. Every test taker starts with the easiest item in the first series, and continues with this series until two incorrect responses have been given. The next item to be administered is an item from the second series, with an item number corresponding to the number of correct responses given in the first series minus one. Again, the second series is continued until two incorrect responses have been given. For those subtests for which a third series is available, the procedure continues analogously, with the missing responses at the start of the series counted as correct responses in the computation of the next item number.

The adaptive procedure has the advantage that fewer items need to be administered to obtain the same accuracy in ability estimates as a standard linear test. Items that would not give much information on the ability of the test taker, because previous responses have shown they are far too easy or too difficult for the test taker, are not administered. As a logical consequence of the adaptive procedure missing data are present. Note, however, that this item administration design can be ignored in data analyses, because the observations completely determine the design (Rubin, 1976). Therefore, as in traditional computerized adaptive testing, inferences can be made ignoring the process causing the missing data.

In the present article, the theories of difficulty for the two spatial subtests will be tested: Mosaics and Patterns. Both subtests consist of two series of respectively 10 and 9 construction items; in total, the tests consist of 20 and 18 items.

1350 Children ( 675 boys and 675 girls) aged between $6-14$ participated in the standardization study (Laros \& Tellegen, 1991; Tellegen \& Laros, 1993). The items were administered in the adaptive procedure described above. The number of (dichotomously scored) responses per item ranged from 92 to 1350 for the Mosaics subtest, with a mean of 814 observations, and from 278 to 1350 for the Patterns subtest, with a mean of 796 observations. For some of the items, the information in the test takers' responses was insufficient to estimate the parameters of the reparameterized $2 \mathrm{PNO}$ model. For example, 90 test takers responded correctly to Item 11 of the Mosaics subtest, whereas only 2 test takers responded incorrectly to this item. However, the sample size did not pose any problems for the two models described above: The L1PNO model has fewer parameters, whereas the L1PNO-C model imposes the informative prior given by (3.5) on the item parameters.

The prior sum of squares $\boldsymbol{S}_{0}$ for the covariance matrix of the L1PNO-C was set to the LICM-C covariance matrix estimates for the Analogies subtest reported by Geerlings et al. (2011; Chapter 2, Table 2.2, model $\left.\boldsymbol{D}_{2}\right)$, i.e., $\operatorname{Vec}\left(\boldsymbol{S}_{0}\right)=$ 
$(0.081,-0.029,0.284)$. The prior degrees of freedom $\nu_{0}$ was set to 2 . For each of the analyses, 300,000 iterations were performed, of which the first 100,000 iterations were discarded as a burn-in. The number of iterations was set very high to ensure that the chains for the effect parameters converged, which was also corroborated by the convergence statistics mentioned above. In the next sections, the item structures and the results of the analyses will be discussed per subtest.

\subsubsection{Item Structures}

\section{Mosaics}

The Mosaics subtest is a special variant of the block design task (Kohs, 1923). To complete an item from this subtest, the test taker is required to copy a redwhite mosaic pattern in a three-by-three grid using different tiles. In the pattern, the border of the tiles is not drawn; it is left to the test taker to figure out its underlying structure.

For the analyses, the design matrix $\boldsymbol{D}$ was constructed using dummy coding of the following difficulty factors. First of all, the types of tiles available to construct the pattern varied per item. In both series of items, for the first two items only completely red and completely white tiles were available. For the third until the seventh items also straight and diagonal half-white/half-red tiles were available, and for the eighth until the tenth items all tiles could be used (including a 'chair'form and an 'hourglass'-form). Also, vertical or horizontal symmetry in the mosaic pattern was expected to result in easier items than asymmetry. Finally, the number of times a color crossed the border between two squares was counted and used as a difficulty factor. An item with many boundary crossings was expected to be more difficult because they obscure the structure of the fields. The counts were recoded into two categories, one representing relatively little boundary crossings, and one representing many boundary crossings.

Figure 3.1(a) presents an example item similar to the items in the subtest Mosaics. All tiles may be used by the test takers to solve the item. The structure of the fields is highly obscured: 88 percent of the boundaries contain the same color on both sides. Therefore, the item is an example of the second category of the number-of-crossings difficulty factor.

\section{Patterns}

Each item from the subtest Patterns consists of one or two repeating line patterns. Part of the image is missing, and the test taker is required to fill in the omission by drawing a continuation of the lines. In doing this, the test taker is helped by a grid drawn over the sheet of paper.

Also in the Patterns subtest, asymmetric patterns were expected to be more difficult than symmetric patterns. A second difficulty factor was the presence of retrograde line parts, which interrupt the progression of a line pattern from left to right. A similar interruption occurs when a line pattern has a large amount of alternation under and above an imaginary base line. Two other difficulty factors were the number of lines (one or two), and whether, in the case of two lines, transection occurred. Also, the size of the omission was expected to influence the 

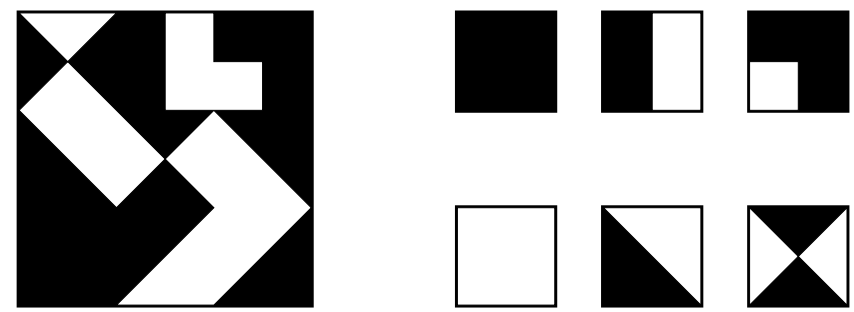

(a) Mosaics

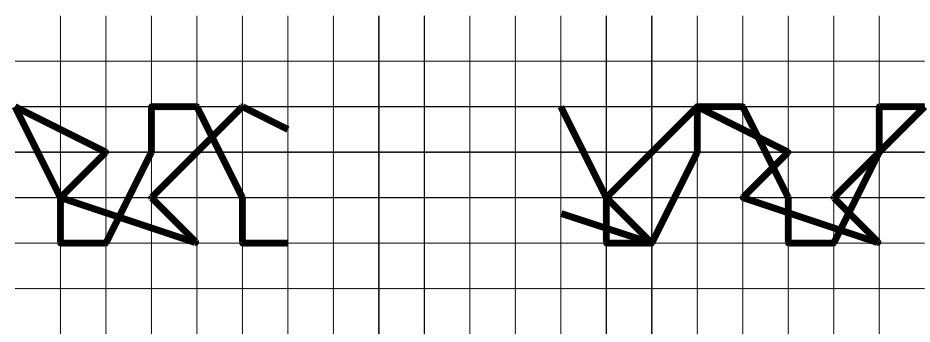

(b) Patterns

Figure 3.1: Example items similar to the items in the SON-R 51/2-17.

difficulty of the items. The sizes were recoded into three categories, with the last category containing the largest sizes.

Figure 3.1(b) presents an example item similar to the items in the subtest Patterns. The figure contains two line patterns, both of which are asymmetric, and transect each other. Also, one of the line patterns contains retrograde line parts. Finally, the size of the omission is larger than the pattern; that is, the item is an example of the third category of the corresponding difficulty factor.

\subsubsection{Results}

\section{Mosaics}

Table 3.1 presents the estimated effects of the difficulty factors $\boldsymbol{\beta}$ (first 6 rows) and the mean discrimination parameter $\mu_{a}$ (last row) for the Mosaics subtest in the L1PNO and L1PNO-C models, respectively. Note that the first difficulty factor, 'Wholes', equals an intercept, because the respective tiles were available for all items. The reported point estimates are EAP estimates and the standard deviations are posterior standard deviations. The difficulty factor estimates that had a $95 \%$ highest posterior density (HPD) interval not including zero are indicated in bold. The probabilities corresponding to the hypotheses tests and the posterior predictive checks are given in Table 3.2. In the table, $P^{a \geq \mu_{a}}$ and $P^{b \geq \mu_{b}}$ refer to the probabilities of respectively an item discrimination or difficulty parameter in the L1PNO-C being larger than its mean. Furthermore, $P^{+}, P^{-}$, and $P^{N A}$ are the posterior predictive p-values for the number of correct, incorrect, and missing 
responses, respectively. For each of the probabilities, a very small or very large value indicates that the targeted assumption (either of a parameter being equal to its mean value, or of replicated item score distributions being equal to the observed item score distribution) is violated.

The L1PNO-C covariance matrix estimates indicated residual variance in the discrimination and difficulty parameters: $\operatorname{Vec}(\boldsymbol{\Sigma})=(0.132,-0.011,0.360)$. The correlation between the item discrimination and difficulty deviations from their means was small: -0.050. Eight items (Items 1, 3, 4, 10, 13, 16, 18, and 19) had an estimated discrimination parameter that deviated significantly from the mean discrimination parameter $\mu_{a}$, which had a value of 1.411 (see $P^{a \geq \mu_{a}}$ in Table 3.2). With regard to residual variance in the difficulty parameters, especially four items (Items 1, 8, 9, and 14) showed large deviations from their mean difficulty parameters. Two of the items with large deviations (Items 1 and 18) had a large impact on the covariance estimate of $\boldsymbol{\Sigma}$, which was negative, whereas the main trend for the other items was positive (see the upper plot in Figure 3.2).

The posterior predictive p-values for the L1PNO model show the effect of these deviations on the fit of the model. The predictions of the model regarding the distribution of the responses differed substantially from the observed distribution. For example, in case of a positive deviation between a difficulty parameter and its mean in the L1PNO-C (Items 1 and 9), replicated data for the L1PNO showed larger numbers of correct responses than the observed data, as indicated by $\mathrm{P}^{+}$. Similarly, in case of negative deviations for the difficulty parameter (Items 8 and 14), replicated data for the L1PNO showed larger numbers of incorrect responses than the observed data, as indicated by $P^{-}$. However, the effects were mediated by the deviations for the discrimination parameters and the adaptive administration procedure (see, for example, the results for Item 19). In general, the model predicted less missing responses in the first series of items, Item 1-10, and more missing responses in the second series of items, Item 11-20.

In contrast, the predictions of the model with random item parameters, the L1PNO-C, regarding the distribution of the responses were close to the observed distribution. The two p-values for $P^{N A}$ equal to one (Items 1 and 2) were caused by the fact that due to the administration procedure every real or simulated test taker responded to the first two items. A side-effect of the more complex L1PNO$\mathrm{C}$ model were larger standard errors, which caused two difficulty factors that were significant in the L1PNO model to be non-significant in the L1PNO-C.

The order of the magnitudes of the difficulty factor estimates were the same for both models. Increasing the number of tiles to choose from made the items substantially more difficult, especially when adding tiles colored in halves. As expected, the two symmetry factors had a negative influence on the difficulty of the items. In the items with a symmetric design, two columns (in the case of vertical symmetry) or two rows (in the case of horizontal symmetry) of the design are the same. Therefore, two-thirds of the item could be solved by detecting the symmetry and solving just one column or row. However, the effect was larger for vertical than for horizontal symmetry. This orientation effect, in which vertical symmetry is more easily detected than horizontal symmetry, has also been described in the literature (Ferguson, 2000). The effect of the number-of-crossings factor is recognized as a 'perceptual cohesiveness' effect (Miller \& Skillman, 2008). 
Table 3.1: Estimated effects of the difficulty factors and the mean discrimination parameter for the Mosaics subtest (and standard deviations).

\begin{tabular}{lrr}
\hline Difficulty factors & L1PNO & L1PNO-C \\
\hline Tiles available & & \\
Wholes & $\mathbf{- 3 . 3 9 6}(0.118)$ & $\mathbf{- 3 . 3 3 0}(0.489)$ \\
Halves & $\mathbf{2 . 5 6 3}(0.112)$ & $\mathbf{2 . 3 6 3}(0.510)$ \\
Chair/hour & $\mathbf{1 . 0 0 9}(0.040)$ & $\mathbf{1 . 5 1 9}(0.387)$ \\
Symmetry & & \\
Vertical & $\mathbf{- 1 . 3 6 1}(0.047)$ & $\mathbf{- 1 . 1 3 1}(0.362)$ \\
Horizontal & $\mathbf{- 0 . 1 7 0}(0.041)$ & $-0.318(0.367)$ \\
No. of crossings & $\mathbf{0 . 7 0 0}(0.041)$ & $0.577(0.399)$ \\
\hline$\mu_{a}$ & $1.195(0.037)$ & $1.411(0.096)$ \\
\hline
\end{tabular}

The boldfaced numbers indicate estimates that had a 95\% HPD interval not including zero.

Table 3.2: Test results for the Mosaics subtest.

\begin{tabular}{lcccccccc}
\hline & \multicolumn{2}{c}{ Hypotheses } & \multicolumn{4}{c}{ Posterior predictive checks } \\
\multicolumn{2}{c}{ L1PNO-C } & \multicolumn{3}{c}{ L1PNO } & \multicolumn{3}{c}{ L1PNO-C } \\
Item & $P^{a \geq \mu_{a}}$ & $P^{b \geq \mu_{b}}$ & $P^{+}$ & $P^{-}$ & $P^{N A}$ & $P^{+}$ & $P^{-}$ & $P^{N A}$ \\
\hline 1 & 0.003 & 0.961 & 0.998 & 0.009 & 1.000 & 0.649 & 0.467 & 1.000 \\
2 & 0.380 & 0.274 & 0.502 & 0.591 & 1.000 & 0.528 & 0.557 & 1.000 \\
3 & 0.024 & 0.764 & 0.980 & 0.028 & 0.540 & 0.497 & 0.531 & 0.652 \\
4 & 0.958 & 0.730 & 1.000 & 0.000 & 0.181 & 0.488 & 0.545 & 0.495 \\
5 & 0.829 & 0.598 & 0.903 & 0.811 & 0.000 & 0.476 & 0.625 & 0.348 \\
6 & 0.141 & 0.888 & 0.999 & 0.971 & 0.000 & 0.476 & 0.605 & 0.396 \\
7 & 0.670 & 0.909 & 0.994 & 0.812 & 0.000 & 0.404 & 0.470 & 0.692 \\
8 & 0.063 & 0.016 & 0.000 & 1.000 & 0.023 & 0.277 & 0.260 & 0.952 \\
9 & 0.736 & 0.991 & 1.000 & 0.000 & 0.819 & 0.511 & 0.240 & 0.834 \\
10 & 0.950 & 0.867 & 0.998 & 0.968 & 0.000 & 0.746 & 0.601 & 0.219 \\
\hline 11 & 0.304 & 0.395 & 0.000 & 0.258 & 1.000 & 0.344 & 0.571 & 0.690 \\
12 & 0.243 & 0.113 & 0.000 & 0.951 & 1.000 & 0.392 & 0.644 & 0.604 \\
13 & 0.016 & 0.210 & 0.000 & 1.000 & 1.000 & 0.639 & 0.575 & 0.329 \\
14 & 0.325 & 0.006 & 0.000 & 1.000 & 0.988 & 0.891 & 0.614 & 0.054 \\
15 & 0.895 & 0.371 & 0.643 & 0.067 & 0.866 & 0.725 & 0.612 & 0.164 \\
16 & 0.959 & 0.587 & 0.000 & 0.000 & 1.000 & 0.259 & 0.326 & 0.912 \\
17 & 0.848 & 0.414 & 0.000 & 0.988 & 1.000 & 0.212 & 0.383 & 0.915 \\
18 & 1.000 & 0.202 & 0.000 & 0.981 & 0.998 & 0.551 & 0.609 & 0.345 \\
19 & 0.001 & 0.130 & 0.947 & 0.000 & 0.998 & 0.516 & 0.683 & 0.251 \\
20 & 0.818 & 0.500 & 0.001 & 0.593 & 0.999 & 0.535 & 0.559 & 0.434 \\
\hline
\end{tabular}




\section{Patterns}

The estimated effects and the model fit results for the Patterns subtest are presented in Tables 3.3 and 3.4, respectively. The EAP estimates of the covariance matrix of the L1PNO-C model were $\operatorname{Vec}(\boldsymbol{\Sigma})=(0.151,-0.225,0.675)$. Hence, the correlation between the item discrimination and difficulty deviations from their means was relatively large: -0.705 . Compared to the results for the Mosaics subtest, the residual variance estimates of the discrimination and especially the difficulty parameters were larger for the Patterns subtest. In particular, eight items (Items 1, 3, 4, 5, 8, 12, 13, and 14) had an estimated discrimination parameter significantly lower or higher than the mean discrimination parameter, which had a value of 1.852 (see $P^{a \geq \mu_{a}}$ in Table 3.4). With regard to residual variance in the difficulty parameters, especially seven items (Items 2, 3, 4, 5, 8, 13, and 14) showed large deviations from their mean difficulty parameters. However, none of the items seemed to be outliers in the negative trend of the deviations; see the lower plot in Figure 3.2.

The posterior predictive p-values for the L1PNO indicated that the predictions of the model with regard to the score distribution per item differed highly from the observed distribution. In many cases, the replicated counts were either almost never or almost always larger than the observed counts. Again, the predictions of the model with random item parameters, L1PNO-C, were close to the observed values.

In general, the models agreed that the addition of a difficulty factor made an item more difficult. However, both the order of the difficulty factor estimates and their absolute magnitudes differed between the models. Similar to the results for the Mosaics subtest, the estimated discrimination parameter $\mu_{a}$ had a lower value in the L1PNO than in the L1PNO-C model.

\subsubsection{Conclusion}

For both subtests, the results of the model fit statistics indicated that the item parameters of the L1PNO model did not describe the discriminatory power and difficulty of the items well. In contrast, the statistics did not show any misfit for the first-level model of the L1PNO-C. Moreover, the results showed a larger amount of residual variance in the item parameters for the Patterns subtest compared to the Mosaics subtest. Also, for the former subtest, larger differences between the L1PNO and L1PNO-C $\boldsymbol{\lambda}$ estimates were found than for the latter subtest. These effects and their implications for testing hypotheses regarding the effect of item construction rules on item difficulty and for ability estimation were further explored in a simulation study.

\subsection{Simulation Study}

The goals of the simulation study were threefold. First, the effects of the difference in fit of the L1PNO and L1PNO-C models to the data of the Mosaics and Patterns subtests on the estimates of $\boldsymbol{\lambda}$ was investigated using replicated data. The two models only differ in their assumptions regarding residual variance in the item 
Table 3.3: Estimated effects of the difficulty factors and the mean discrimination parameter for the Patterns subtest (and standard deviations).

\begin{tabular}{lrr}
\hline Difficulty factors & L1PNO & L1PNO-C \\
\hline Intercept & $\mathbf{- 3 . 2 8 2}(0.092)$ & $\mathbf{- 4 . 4 4 0}(0.534)$ \\
Asymmetric patterns & $\mathbf{0 . 6 8 7}(0.034)$ & $\mathbf{1 . 2 6 5}(0.338)$ \\
Retrograde line parts & $\mathbf{0 . 8 9 2}(0.038)$ & $\mathbf{1 . 6 9 0}(0.439)$ \\
Alternation & $\mathbf{1 . 4 4 6}(0.054)$ & $\mathbf{1 . 3 4 3}(0.562)$ \\
Number of lines & $\mathbf{0 . 2 9 5}(0.089)$ & $0.248(0.847)$ \\
Transection & $\mathbf{2 . 4 7 9}(0.064)$ & $\mathbf{3 . 2 8 5}(0.642)$ \\
Size of the omission & $\mathbf{0 . 8 1 1}(0.073)$ & $0.867(0.537)$ \\
\hline$\mu_{a}$ & $1.377(0.042)$ & $1.852(0.109)$ \\
\hline
\end{tabular}

The boldfaced numbers indicate estimates that had a 95\% HPD interval not including zero.

Table 3.4: Test results for the Patterns subtest.

\begin{tabular}{lcccccccc}
\hline & \multicolumn{2}{c}{ Hypotheses } & \multicolumn{4}{c}{ Posterior predictive checks } \\
\multicolumn{3}{c}{ L1PNO-C } & \multicolumn{3}{c}{ L1PNO } & \multicolumn{3}{c}{ L1PNO-C } \\
Item & $P^{a \geq \mu_{a}}$ & $P^{b \geq \mu_{b}}$ & $P^{+}$ & $P^{-}$ & $P^{N A}$ & $P^{+}$ & $P^{-}$ & $P^{N A}$ \\
\hline 1 & 0.003 & 0.879 & 0.000 & 1.000 & 1.000 & 0.454 & 0.636 & 1.000 \\
2 & 0.589 & 0.039 & 0.000 & 1.000 & 1.000 & 0.432 & 0.606 & 1.000 \\
3 & 0.010 & 0.999 & 1.000 & 0.000 & 1.000 & 0.541 & 0.464 & 0.626 \\
4 & 0.958 & 0.049 & 0.017 & 0.734 & 0.994 & 0.430 & 0.539 & 0.602 \\
5 & 0.004 & 0.985 & 1.000 & 0.000 & 0.000 & 0.422 & 0.675 & 0.349 \\
6 & 0.785 & 0.497 & 0.992 & 0.847 & 0.000 & 0.453 & 0.487 & 0.617 \\
7 & 0.154 & 0.401 & 0.000 & 1.000 & 0.000 & 0.306 & 0.478 & 0.766 \\
8 & 0.012 & 0.961 & 1.000 & 0.000 & 0.027 & 0.558 & 0.381 & 0.642 \\
9 & 0.326 & 0.833 & 1.000 & 0.000 & 0.873 & 0.536 & 0.106 & 0.920 \\
\hline 10 & 0.681 & 0.358 & 0.000 & 0.994 & 1.000 & 0.377 & 0.501 & 0.682 \\
11 & 0.469 & 0.729 & 0.094 & 0.002 & 1.000 & 0.625 & 0.754 & 0.202 \\
12 & 0.978 & 0.170 & 0.000 & 0.596 & 1.000 & 0.733 & 0.533 & 0.234 \\
13 & 0.999 & 0.016 & 0.015 & 0.983 & 0.740 & 0.613 & 0.522 & 0.379 \\
14 & 0.983 & 0.015 & 0.000 & 1.000 & 0.021 & 0.791 & 0.656 & 0.093 \\
15 & 0.634 & 0.253 & 0.000 & 0.275 & 1.000 & 0.197 & 0.494 & 0.895 \\
16 & 0.323 & 0.787 & 0.000 & 0.052 & 1.000 & 0.485 & 0.476 & 0.600 \\
17 & 0.170 & 0.659 & 0.999 & 0.000 & 1.000 & 0.544 & 0.457 & 0.560 \\
18 & 0.828 & 0.175 & 0.001 & 0.000 & 1.000 & 0.496 & 0.287 & 0.774 \\
\hline
\end{tabular}



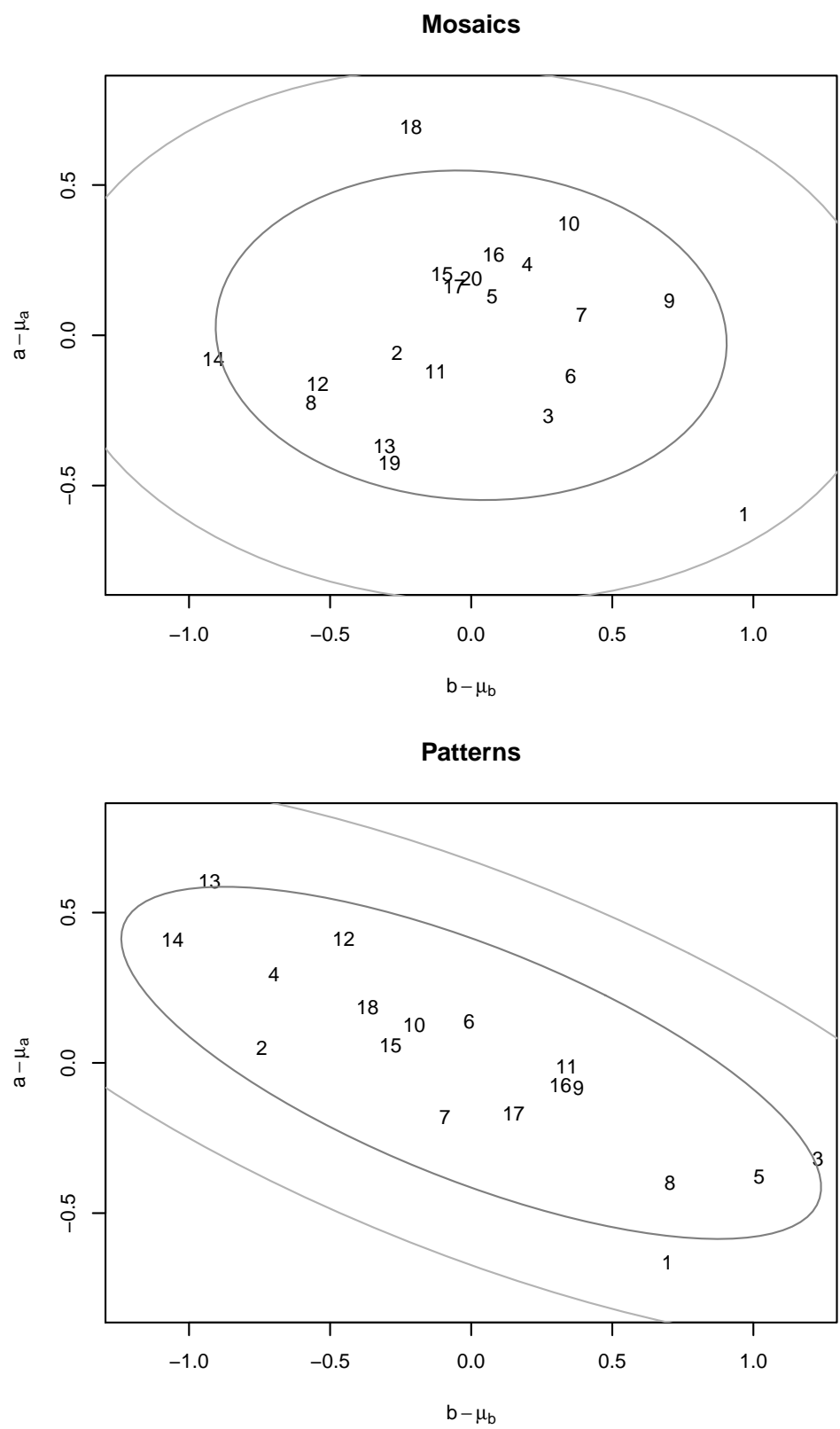

Figure 3.2: Deviations of the L1PNO-C item parameters from their mean values and $68 \%$ and $95 \%$ confidence ellipsoids based on the estimated covariance matrix. 
parameters: The L1PNO assumes that it does not exist, whereas the L1PNO$\mathrm{C}$ assumes it is multivariate normal distributed. The results of the model fit assessment of the empirical study showed that for both subtests the assumption of a perfect prediction by the mean discrimination and difficulty factor parameters $\boldsymbol{\lambda}$ did not hold. However, the fit statistics did not give information as to the degree to which the estimates of $\boldsymbol{\lambda}$ were biased by the model violation. Also, whereas the statistics indicated that the L1PNO-C model fitted well on the item level, they were not indicative with regard to its (second-level) assumption of multivariate normality. Therefore, for both subtests, the expected bias for the $\boldsymbol{\lambda}$ estimates was investigated using data simulated under the estimated first-level model of the L1PNO-C.

The other two goals of the study were to investigate the use of the L1PNO and L1PNO-C models for testing hypotheses regarding the effect of item construction rules on item difficulty and for ability estimation in the presence of residual variance in the item parameters. With regard to hypothesis testing, the results of the empirical study indicated a larger difference in the L1PNO and L1PNO-C model estimates of $\boldsymbol{\lambda}$ for the subtest with a larger amount of residual variance in the item parameters (the Patterns subtest). Therefore, the second goal of the simulation study was to further investigate the effect of the amount of residual variance on the robustness of the $\boldsymbol{\lambda}$ estimates of both models using data simulated under the second-level item models of the L1PNO-C and by varying the values for the covariance matrix $\boldsymbol{\Sigma}$.

With regard to ability estimation, the precision of the estimates depends on the mean discrimination parameter. Hence, any bias in the estimates of this parameter can be expected to influence the precision of the ability estimates. Therefore, the third goal of the simulation study was to investigate the effect of model choice on ability estimates obtained using (3.14) and their standard deviations.

\subsubsection{Study Setup}

For the investigation of the expected bias in the empirical study, the L1PNO-C estimates of the person and item parameters of the Mosaics and Patterns subtests were used to replicate datasets. In each of 20 replications, data were generated according to the item selection procedure described in Section 3.3. Subsequently, the parameters were re-estimated under the L1PNO and L1PNO-C model, using the same priors as specified for the empirical study. To reduce the total running time for the study, the true values of the parameters were used as starting values to create a smaller number of burn-in iterations. For each of the runs 50,000 iterations were performed, of which the first 10,000 iterations were discarded as a burn-in. The mean bias and standard deviations of the effect parameters were computed over replications.

For the investigation of the effect of increasing residual variance in the item parameters on the $\boldsymbol{\lambda}$ estimates the procedure was repeated with the following adaptations. In each of the 20 replications, the parameters of either 20 or 18 items were simulated, depending on the subtest, from a multivariate normal distribution with their respective estimated L1PNO-C family means and one of the following covariance matrices: 
1. Matrix equal to zero, i.e. $\operatorname{Vec}(\boldsymbol{\Sigma})=(0,0,0)$,

2. Matrix of the Mosaics subtest, i.e. $\operatorname{Vec}(\boldsymbol{\Sigma})=(0.132,-0.011,0.360)$,

3. Matrix of the Patterns subtest, i.e. $\operatorname{Vec}(\boldsymbol{\Sigma})=(0.151,-0.225,0.675)$.

Note that across these three matrix conditions (MC) the values for the covariance matrix increase in size. Negative draws for the discrimination parameters were discarded. Using the estimated person parameters and the simulated item parameters, data were generated according to the item selection procedure described in Section 3.3. Subsequently, the parameters were re-estimated under the L1PNO and L1PNO-C model, using the same priors as specified for the empirical study. The three matrix conditions crossed with the two subtests (i.e., ability parameters, family means, design matrix and number of items fixed at the empirical values) resulted in six conditions. Again, the true values of the parameters were used as starting values, except for the first $\mathrm{MC}$ in which $(0.01,0,0.01)$ was used as starting values for the covariance matrix to avoid singularity.

For the investigation of the effect of residual variance in the parameters on ability estimates, one dataset was simulated for each of the two subtests in a manner similar to the one described above for the first part of the simulation study. That is, the EAP estimates of the item and ability parameters of the empirical study were used in combination with the item selection procedure to generate two datasets. Ability was estimated using (3.14) and either of the following:

1. Estimated item parameters of the L1PNO-C model, $\boldsymbol{\xi}$,

2. Estimated effect parameters of the L1PNO model, $\boldsymbol{\lambda}$,

3. Estimated higher-level parameters of the L1PNO-C model, $\boldsymbol{\lambda}$ and $\boldsymbol{\Sigma}$.

The first condition assumed calibration of every item and was used as a baseline. The second and third conditions are for the cases in which the item parameters are assumed unknown, but the parameters $\boldsymbol{\lambda}$ (L1PNO) or $\boldsymbol{\lambda}$ and $\boldsymbol{\Sigma}$ (L1PNO-C) have been calibrated. The integrals in (3.13) were approximated using 5000 Monte Carlo iterations and the integral in (3.14) using 15 Gauss-Hermite quadratures. Although the use of only one replication limited the generalizability of the results of this part of the study, it allowed for a closer inspection of the ability estimates and their standard deviations.

\subsubsection{Results}

In Tables 3.5 (Mosaics subtest) and 3.6 (Patterns subtest), the mean bias and standard deviations for the replication study with the item parameters fixed at their empirical values are presented. To give an indication of the significance of the results, t-tests for independent samples were performed on the bias and standard deviations of the 20 replications. The values that were significantly different between the L1PNO and L1PNO-C model at the 0.05 level are indicated in bold.

A comparison of the bias for both models as applied to the Patterns subtest showed a substantial improvement of the L1PNO-C over the L1PNO (Table 3.6). 
Table 3.5: Mean bias (and mean standard deviations) for the estimated effects of the difficulty factors and the mean discrimination parameter for the Mosaics subtest based on 20 replicated datasets.

\begin{tabular}{lrr}
\hline Difficulty factors & L1PNO & L1PNO-C \\
\hline Tiles available & & \\
Wholes & $\mathbf{- 0 . 1 8 2}(\mathbf{0 . 1 2 5})$ & $\mathbf{- 0 . 5 3 6}(\mathbf{0 . 4 2 4})$ \\
Halves & $\mathbf{0 . 3 0 1}(\mathbf{0 . 1 1 9 )}$ & $\mathbf{0 . 4 8 7}(\mathbf{0 . 4 4 4 )}$ \\
Chair/hour & $\mathbf{- 0 . 4 9 4}(\mathbf{0 . 0 4 1})$ & $\mathbf{- 0 . 1 8 9}(\mathbf{0 . 3 1 0})$ \\
Symmetry & & \\
Vertical & $-0.243(\mathbf{0 . 0 4 6 )}$ & $-0.232(\mathbf{0 . 2 9 5})$ \\
Horizontal & $0.150(\mathbf{0 . 0 4 0 )}$ & $0.140(\mathbf{0 . 3 0 2})$ \\
No. of crossings & $0.095(\mathbf{0 . 0 4 1})$ & $0.134(\mathbf{0 . 3 2 1})$ \\
\hline$\mu_{a}$ & $\mathbf{- 0 . 2 4 5}(\mathbf{0 . 0 3 7})$ & $\mathbf{- 0 . 0 2 1}(\mathbf{0 . 0 8 0})$ \\
\hline
\end{tabular}

The boldfaced numbers indicate significant differences in bias or standard deviations between the L1PNO and L1PNO-C model at the 0.05 level.

Table 3.6: Mean bias (and mean standard deviations) for the estimated effects of the difficulty factors and the mean discrimination parameter for the Patterns subtest based on 20 replicated datasets.

\begin{tabular}{lrr}
\hline Difficulty factors & L1PNO & L1PNO-C \\
\hline Intercept & $\mathbf{1 . 1 6 8}(\mathbf{0 . 0 9 2})$ & $\mathbf{- 0 . 0 4 6}(\mathbf{0 . 5 1 4})$ \\
Asymmetric patterns & $\mathbf{- 0 . 5 7 8}(\mathbf{0 . 0 3 5})$ & $\mathbf{0 . 0 5 9}(\mathbf{0 . 3 4 9})$ \\
Retrograde line parts & $\mathbf{- 0 . 7 8 5}(\mathbf{0 . 0 3 9})$ & $\mathbf{- 0 . 0 4 6}(\mathbf{0 . 4 3 6})$ \\
Alternation & $\mathbf{0 . 0 6 1}(\mathbf{0 . 0 5 3})$ & $\mathbf{- 0 . 0 8 8}(\mathbf{0 . 5 8 0})$ \\
Number of lines & $\mathbf{0 . 0 1 9}(\mathbf{0 . 0 8 9})$ & $\mathbf{- 0 . 1 7 1}(\mathbf{0 . 8 3 0})$ \\
Transection & $\mathbf{- 0 . 7 6 9 ( 0 . 0 6 5 )}$ & $\mathbf{- 0 . 1 2 4}(\mathbf{0 . 6 4 7})$ \\
Size of the omission & $\mathbf{- 0 . 0 6 7}(\mathbf{0 . 0 7 3})$ & $\mathbf{0 . 0 8 9}(\mathbf{0 . 5 3 3})$ \\
\hline$\mu_{a}$ & $\mathbf{- 0 . 5 2 1}(\mathbf{0 . 0 4 0})$ & $\mathbf{- 0 . 0 6 5 ( \mathbf { 0 . 1 0 3 } )}$ \\
\hline
\end{tabular}

The boldfaced numbers indicate significant differences in bias or standard deviations between the L1PNO and L1PNO-C model at the 0.05 level.

Especially for the intercept, three of the difficulty factors, and the mean discrimination parameter of the L1PNO model the bias was large. The bias for the L1PNO model applied to the Mosaics subtest was smaller, but still substantial for some of the difficulty factors (Table 3.5). For this subtest however, the L1PNO-C model also showed large bias for the difficulty factor estimates, in particular for the intercept. We expect these divergent results to be due to the two apparent outlying Items 1 and 18 in the Mosaics subtest (see the upper plot in Figure 3.2), which influenced the estimates of the covariance matrix.

The results for the study of the effect of the values of the covariance matrix on 
$\boldsymbol{\lambda}$ are presented in Tables 3.7 (Mosaics subtest) and 3.8 (Patterns subtest). In all cases, the standard deviations for the estimates of the more complex L1PNO-C model were larger than for the L1PNO estimates. In particular, as opposed to the standard deviations for the L1PNO-C model, the standard deviations for the L1PNO model remained approximately the same with values for the covariance matrix increasing in size. As can be seen from (A.15) in the Appendix, the variance in the conditional distribution of $\boldsymbol{\lambda}$ is independent of $\boldsymbol{\Sigma}$ in the L1PNO. However, the variance of $\boldsymbol{\lambda}$ in the L1PNO-C depends on $\boldsymbol{\Sigma}$; see (A.23).

On the other hand, the bias for the L1PNO discrimination and difficulty factor estimates increased with values for $\boldsymbol{\Sigma}$ increasing in size. With regard to the discrimination parameter, the last row of Tables 3.7 and 3.8 show an increasing negative bias across matrix conditions. Previously, a similar effect has been found by Bradlow, Wainer, and Wang (1999) in the context of a model for items grouped under a common stimulus. These authors explain their finding as follows: “(...) a is a measure of the correlation of the item with the underlying trait, if the model fits less well (there is more noise), this correlation will be smaller." (p. 164).

With regard to the larger difficulty factor parameters, the bias increased inward across matrix conditions. This effect is a logical consequence of the bias in the discrimination parameter and the reparameterization of the model, with $a_{i} \theta_{n}-b_{i}$ as the argument of $\Phi($.$) . In this parameterization, b_{i}$ equals $a_{i} b_{i}$ of the parameterization in (3.2). Hence, any negative bias in the discrimination parameter resulted in an inward bias in the difficulty factor parameters.

Figures 3.3 (Mosaics subtest) and 3.4 (Patterns subtest) show the results for the simulated example of the effect of residual variance in the item parameters on the L1PNO and L1PNO-C estimates of $\boldsymbol{\theta}$ (left-hand columns) and their standard deviations (right-hand columns). The estimates computed from (3.14) given the item parameters $\boldsymbol{\xi}$ of the L1PNO-C model functioned as a baseline. The first and second rows of the figures show these baseline estimates plotted against those based on the $\boldsymbol{\lambda}$ estimates of the L1PNO and the $\boldsymbol{\lambda}$ and $\boldsymbol{\Sigma}$ estimates of the L1PNO$\mathrm{C}$ model, respectively. The third row is for the comparison of the latter two types of estimates.

For the Patterns subtest, the ability estimates were very similar across the three model conditions. However, for the Mosaics subtest, the estimates based on the $\boldsymbol{\lambda}$ and $\boldsymbol{\Sigma}$ estimates of the L1PNO-C model were larger on the lower end of the scale than those based on its first-level item parameters $\boldsymbol{\xi}$ (second row and first column of Figure 3.3). In general, the standard deviations of the ability estimates were larger for both estimation based on $\boldsymbol{\lambda}$ (L1PNO) and $\boldsymbol{\lambda}$ and $\boldsymbol{\Sigma}$ (L1PNO-C) than for estimation based on the individual item parameters $\boldsymbol{\xi}$ of the L1PNO-C. However, a comparison of the standard deviations for the former two (last row and second column of Figures 3.3 and 3.4) showed differences in their exact values.

\subsubsection{Conclusion}

Whereas for the L1PNO-C unexplained variance in the item parameters resulted in larger standard deviations for the effect parameters, for the L1PNO it resulted in an inward bias in these parameters. In both cases, the significance of the effect parameters can be expected to decrease. However, the L1PNO-C model yielded 


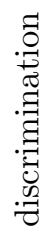

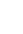

g 곻

궁

范

곤

पै

on

电

궁

冚.

है

范

需

.

की.

衰

.

过

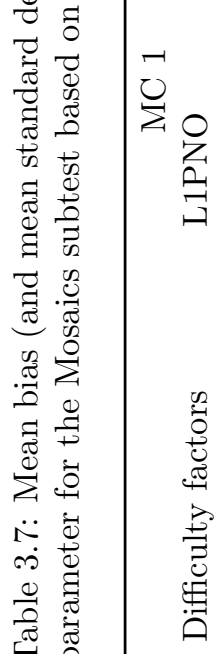

$\infty$

$\circlearrowright$

z

$\mapsto$

.

实

O

.

8

8

फ

웡

己

$\stackrel{2}{0}$

0
1
0
7
$ㄱ$
1 \begin{tabular}{l}
0 \\
1 \\
0 \\
7 \\
01 \\
7 \\
\hdashline
\end{tabular}

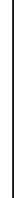

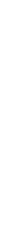

เกิโลิกลิก

O̊

$\dot{\theta} \dot{\theta} \dot{\theta} \quad \dot{\theta} \dot{\theta} \dot{\theta} \dot{\theta}$

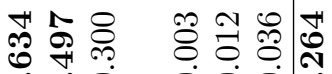

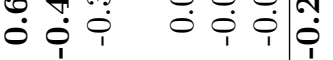

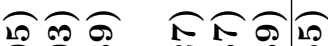

ำ ำ ำ

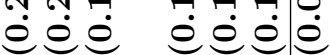

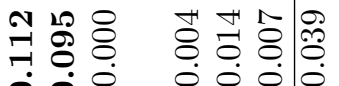

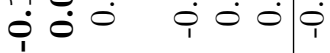

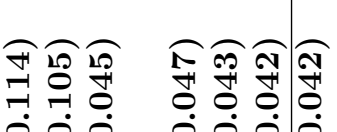

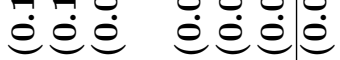

당

ب̂

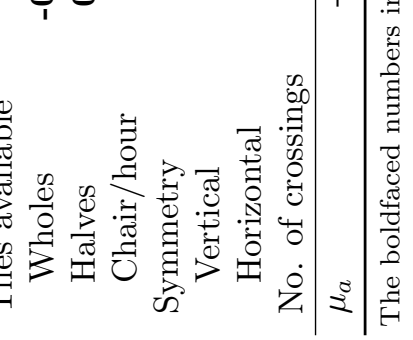

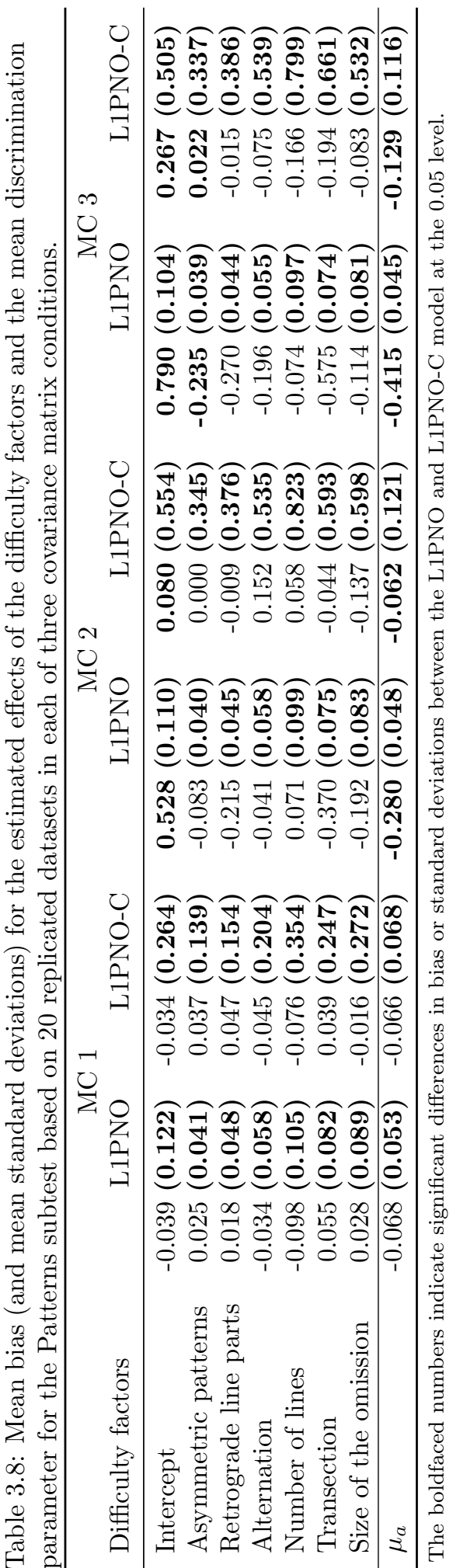



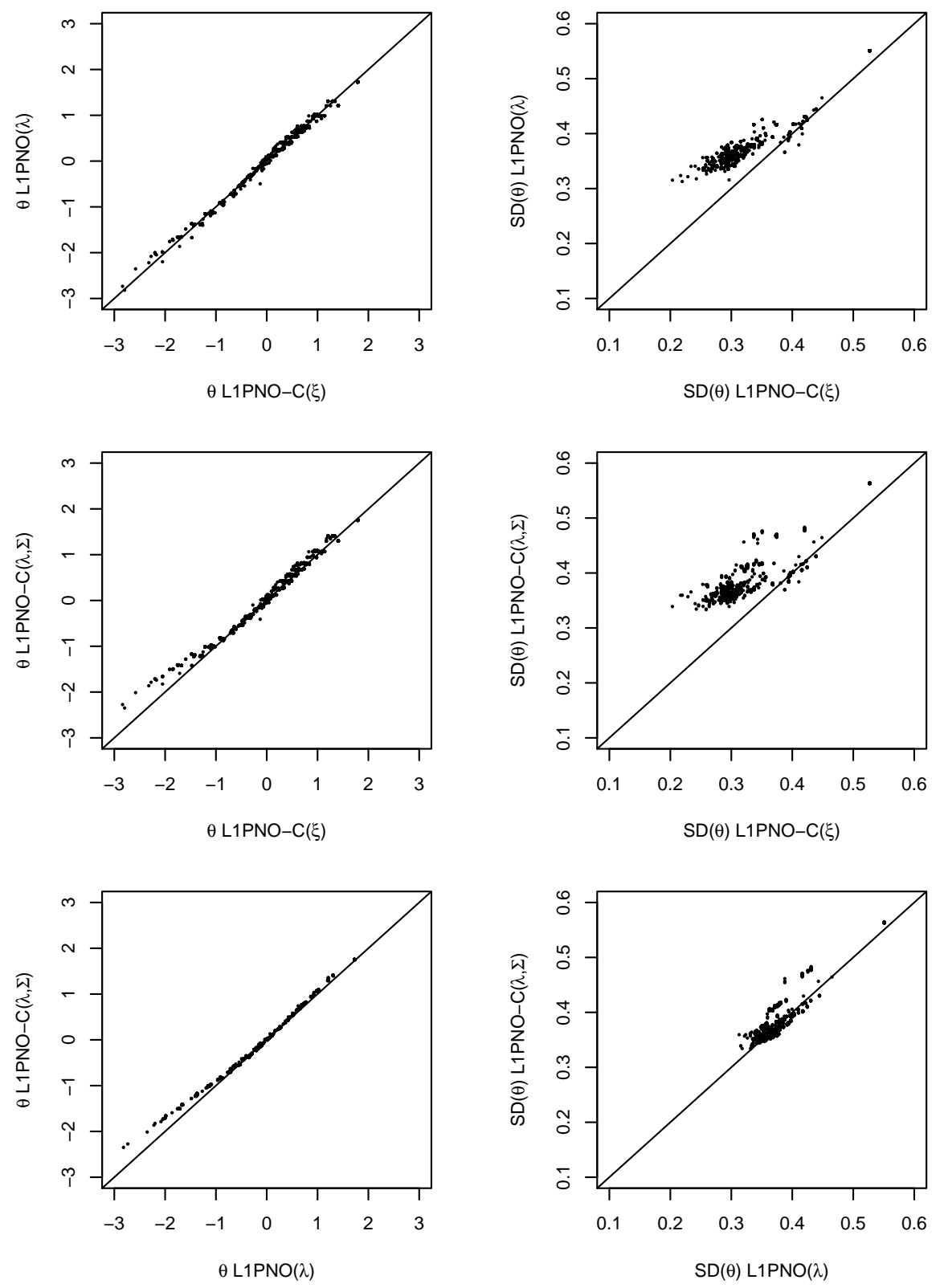

Figure 3.3: Comparisons of the ability estimates (left column) and standard deviations (right column) computed using the effect parameters $\boldsymbol{\lambda}$ of the L1PNO and the item $(\boldsymbol{\xi})$ or second-level parameters $(\boldsymbol{\lambda}, \boldsymbol{\Sigma})$ of the L1PNO-C model for the Mosaics subtest. 

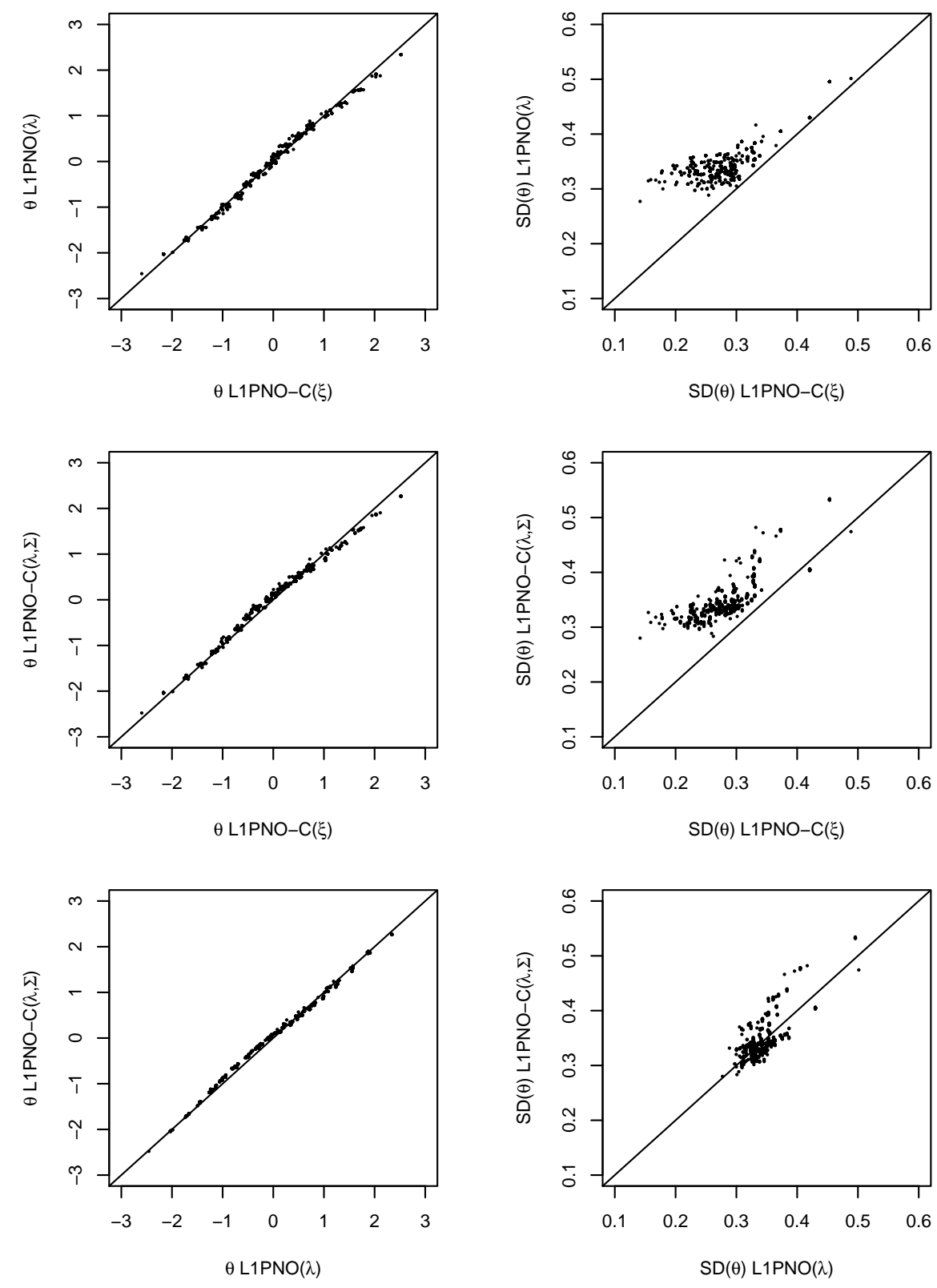

Figure 3.4: Comparisons of the ability estimates (left column) and standard deviations (right column) computed using the effect parameters $\boldsymbol{\lambda}$ of the L1PNO and the item $(\boldsymbol{\xi})$ or second-level parameters $(\boldsymbol{\lambda}, \boldsymbol{\Sigma})$ of the L1PNO-C model for the Patterns subtest. 
more conservative conclusions.

Similarly, for both the L1PNO and the L1PNO-C model, the standard deviations of ability estimates increased with increasing residual variance in the item parameters. For the L1PNO, the increase was due to a negative bias in the estimated discrimination parameter, whereas for the L1PNO-C it was due to explicit modeling of the residual variance.

\subsection{Discussion}

Constructing items based on a feature model for item difficulty has several advantages. First of all, using IRT models with a structural model on the item parameters, hypotheses regarding the cognitive processes involved in solving the items can be tested while taking into account individual ability levels.

A second advantage is that, after calibration of the difficulty factor parameters, it is possible to construct new items within a prespecified range of difficulty. In the context of adaptive testing, this allows for the generation of items adapted to test takers' ability during test taking (so called item generation on-the-fly).

To account for the uncertainty in the item parameters due to prediction based on item features instead of single-item calibration, a random effects model such as the L1PNO-C model discussed in this article can be used to estimate the difficulty factor and ability parameters. The random components in the model encompass all variation not accounted for by the design matrix.

Ignoring residual variance in the item parameters, as in the L1PNO, resulted in a negative bias in the mean discrimination parameter and a corresponding inward bias in the difficulty factor parameters. Although the bias resulted in more conservative conclusions with regard to hypothesis testing and ability estimation, the results for the L1PNO-C tended to be even more so. The L1PNO-C also takes into account that the deviations of the discrimination and difficulty parameters from their mean values imposed by the structural model may be correlated. In our studies, we did not investigate the effects of variances and covariances separately, which may be an area for further research.

The results of the studies also suggested that the advantages of explicit modeling of residual variance may not hold when an incorrect distribution for the (co)variances is assumed. The first-level fit statistics discussed in this article are insufficient to detect such misfit, but in Chapter 4 the topic of second-level model fit assessment is discussed in detail. If such an assessment shows that the multivariate normal distribution cannot accurately describe the residual variance in the item parameters, alternative distributions can be considered. 


\title{
Chapter 4
}

\section{Assessing Item-Family Model Fit}

\begin{abstract}
Bayesian fit statistics are discussed for a range of item response theory models for clusters of items created through item cloning. The statistics can be used to assess whether the psychometric properties of the item clusters can be explained by covariates, and whether the variability of item parameters within clusters is accurately represented by the covariance structure of the models. The statistics are embedded in extended posterior predictive checks (Gelman et al., 2005), which entails a comparison of replicated (latent) data with "observed" (latent) data. The methodology is illustrated using a dataset on statistical word problems generated by systematically applied construction rules.
\end{abstract}

Key words: (extended) posterior predictive check, item generation, item response theory, hierarchical modeling, model fit, statistical word problems.

\subsection{Introduction}

Generating assessment items based on clearly defined construction rules has the potential for automation of the item-generation process. In particular, if the cognitive processes underlying item solving can be made explicit, rules can be defined that either target these processes (so called 'radicals'; Irvine, 2002) or do not (so called 'incidentals'; Irvine, 2002). The radicals can be expected to influence psychometric properties of the generated items, such as their difficulty and discriminatory power. In contrast, the incidentals are expected to result only in minor random variation of such properties. The combined use of radicals and incidentals in item generation results in sets or 'families' of items with similar psychometric properties. Between-family variation is then caused by the different combinations of radicals, whereas within-family variation is caused by the incidentals.

This chapter has been written in collaboration with C. A. W. Glas, H. Holling, W. J. van der Linden, and N. Zeuch. 
In Glas and van der Linden's (2003) item cloning model (ICM), the clustering of items into families is accounted for by means of a multilevel structure on the item parameters of an IRT model. In the ICM, the item parameters are assumed to be multivariate normally distributed within their respective families. That is, each item parameter is equal to its family mean parameter, plus an item-specific deviation. Various restrictions and generalizations can be applied to the model to test assumptions about the effect of the radicals on the psychometric properties of the item families, and the effect of the incidentals on the variability of the item parameters within families (Geerlings et al., 2011; Chapter 2). Comparisons between models with different restrictions can give insight into the validity of the hypothesized structure of the test.

Most existing fit statistics focus on either item or person fit. However, in an assessment of the effect of the radicals and the incidentals on the psychometric properties of the items, the fit of the family distributions is of primary interest. As these are distributions of latent variables, a model fit assessment should ideally take into account that the latent variables are estimated with some error. By using extended posterior predictive checks (Gelman et al., 2005; Steinbakk \& Storvik, 2009), a Bayesian approach to model fit assessment, both the uncertainty regarding the item and the family parameters is taken into account. In this article, a range of fit statistics will be considered that target specific assumptions regarding the family distributions. The statistics can be used in the selection of a structural model that is able to describe the item families well. Furthermore, item and family expected response functions (Lewis, 2001; Sinharay et al., 2003) will be used to visualize the results of a selected model.

The general modeling approach will be discussed in more detail in the next section. In Section 4.3, the methodology will be illustrated with an application on statistical word problems (Zeuch, 2011). The items were systematically generated through the combined use of radicals targeted at the statistical formulas underlying the questions, and incidentals in the form of different context stories in which the questions were embedded. The article will end with a discussion on the model complexity-model fit trade-off in the selection of a model for automatically generated items.

\subsection{Modeling Approach}

Both the general item cloning model and the restricted versions will be discussed in Section 4.2.1. Details on the estimation procedure and the model fit measures used will be given in Sections 4.2.2 and 4.2.3, respectively. Finally, in Section 4.2.4 the item and family expected response functions will be described.

\subsubsection{Response Models}

By means of a multilevel structure, the model of Glas and van der Linden (2003) takes into account grouping of the items in families. Let $\boldsymbol{\xi}_{i_{f}}=\left(a_{i_{f}}, b_{i_{f}}\right)$ denote the item parameters of item $i_{f}, i_{f}=1, \ldots, I_{f}$, in family $f, f=1, \ldots, F$, where $a_{i_{f}}$ refers to the discriminatory power of the item, and $b_{i_{f}}$ to its difficulty. Glas 
and van der Linden (2003) presented the ICM with the inclusion of guessing parameters; however, these parameters will be omitted from the discussion because guessing was expected not to be an issue for the open-ended word problems in the empirical study reported below. Furthermore, let $\theta_{n_{g}}$ denote the ability of person $n_{g}, n_{g}=1, \ldots, N_{g}$, in group $g, g=1, \ldots, G$, and $U_{i_{f} n_{g}}$ be a dichotomous variable for the response of this person to item $i_{f}$. The symbol $\Phi($.$) will be used to denote$ the normal cumulative density function. Although the logistic function was used in Glas and van der Linden (2003), use of the normal cumulative density function simplifies estimation in a Bayesian framework (see Section 4.2.2 and Appendix A). The first-level model is the two-parameter normal ogive (2PNO) model given by

$$
P\left(U_{i_{f} n_{g}}=1 \mid \theta_{n_{g}}, \boldsymbol{\xi}_{i_{f}}\right)=\Phi\left(a_{i_{f}} \theta_{n_{g}}-b_{i_{f}}\right),
$$

which we extend with an ability distribution for $g>1$ :

$$
\theta_{n_{g}} \sim N\left(\mu_{\theta_{g}}, \sigma_{\theta_{g}}^{2}\right)
$$

The ability distribution for $g=1$ will be fixed at $N(0,1)$ to identify the model. The item parameters of item $i_{f}$ of family $f, f=1, \ldots, F$, are assumed to have a multivariate normal distribution

$$
\boldsymbol{\xi}_{i_{f}} \sim M V N\left(\boldsymbol{\mu}_{f}, \boldsymbol{\Sigma}_{f}\right)
$$

with $\boldsymbol{\mu}_{f}=\left(\mu_{a_{f}}, \mu_{b_{f}}\right)$ the parameters for the mean discrimination and difficulty of family $f$, respectively, and $\boldsymbol{\Sigma}_{f}$ the covariance matrix for the variability of the item parameters around these means.

More parsimonious models can be obtained if certain assumptions regarding the within-family item-parameter variability hold. First, if the variability of the item parameters within families can be assumed to be equal across families, (4.3) can be replaced by

$$
\boldsymbol{\xi}_{i_{f}} \sim M V N\left(\boldsymbol{\mu}_{f}, \boldsymbol{\Sigma}\right)
$$

where $\boldsymbol{\Sigma}$ is a covariance matrix common to all families. If the incidentals are applied equally to all families, the assumption entails that there is no interaction between the incidentals and the families with regard to within-family item-parameter variability.

Second, a more restrictive assumption is the one of the item parameters being exactly equal to their respective family mean parameters:

$$
P\left(U_{i_{f} n_{g}}=1 \mid \theta_{n_{g}}, \boldsymbol{\mu}_{f}\right)=\Phi\left(\mu_{a_{f}} \theta_{n_{g}}-\mu_{b_{f}}\right) .
$$

Such models have been referred to as identical siblings models (Sinharay et al., 2003), as they assume identical item parameters within a family.

Apart from restrictions on the within-family item-parameter variability, the ICM can be restricted by using available information on the radicals used to generate the items. In particular, using models with a linear structure on the family means, it can be investigated whether the difficulty and discriminatory power of the families can be explained by the radicals, and possibly their interaction effects. 
To facilitate the comparison with the models discussed below, consider the following general regression notation for $\mu_{a_{f}}$ and $\mu_{b_{f}}$ :

$$
\begin{aligned}
& \mu_{a_{f}}=\boldsymbol{X}_{f}^{a} \boldsymbol{\lambda}^{a}, \\
& \mu_{b_{f}}=\boldsymbol{X}_{f}^{b} \boldsymbol{\lambda}^{b},
\end{aligned}
$$

where $\boldsymbol{X}^{a}=\left(\boldsymbol{X}_{f}^{a}\right)$ and $\boldsymbol{X}^{b}=\left(\boldsymbol{X}_{f}^{b}\right)$ are design matrices relating the parameters for the mean difficulty and discrimination of the families to vectors with regression parameters $\boldsymbol{\lambda}^{a}$ and $\boldsymbol{\lambda}^{b}$. Apart from the above mentioned assumptions regarding within-family item-parameter variability, all models in this section are identified by the definition of matrices $\boldsymbol{X}^{a}$ and $\boldsymbol{X}^{b}$ in (4.6) and (4.7). For the models introduced thus far, both $\boldsymbol{X}^{a}$ and $\boldsymbol{X}^{b}$ are identity matrices, and $\boldsymbol{\lambda}$ is a $2 \times F$ vector containing the parameters for the mean discrimination and difficulty of the families, respectively.

The effect of the radicals on the mean difficulty of the families can be investigated using a model in which the respective parameters are restricted to be equal to a sum of the radical main effects and possibly also their interaction effects. In this case, $\boldsymbol{X}^{b}$ equals a design matrix relating the families to the radicals used to generate items from them, and $\boldsymbol{\lambda}^{b}$ is a vector with the main effect and interaction parameters.

Unlike the effects of the radicals on the difficulty parameters, mostly we have no precise hypotheses as to their effect on the discriminatory power of the items. However, as noted by Embretson (2005), it may be worthwhile to investigate their effect on this item parameter as well. The respective restrictions can be imposed on the ICM in a manner analogous to the one for the difficulty parameters above. The most restrictive assumption regarding the family discrimination parameters is equality across all families. The restriction is applied to the model by setting $\boldsymbol{X}^{a}$ equal to an array of ones, so that $\lambda^{a}$ becomes a single family discrimination parameter. If all restrictions are applied to the ICM simultaneously, a model similar to Fischer's (1973) LLTM is obtained.

\subsubsection{Parameter Estimation}

In the studies reported below, the normal-ogive models were estimated in a Bayesian manner, using Gibbs sampling algorithms programmed in the software environment R (R Development Core Team, 2009). The Gibbs sampling method entails iteratively drawing from the posterior distributions of subsets of parameters conditional on the values of the other parameters. It can be shown that the resulting set of draws converges to the joint posterior distribution of the parameters (see, for example, Casella \& George, 1992).

The method can be implemented using variations on the data-augmented algorithm presented in Albert (1992). In this context, data augmentation amounts to a transformation of the discrete observed data to continuous latent data. It has the advantage of giving the conditional distributions of the parameters standard forms that are easy to sample. A detailed description of the algorithms is given in Appendix A.1 for the identical siblings models, and in Appendix A.2 for the item cloning models. 
The conjugate prior for $\mu_{\theta_{g}}$ and $\sigma_{\theta_{g}}^{2}$ in (4.2) is the normal inverse chi-square distribution:

$$
\begin{aligned}
\sigma_{\theta_{g}}^{2} & \sim \text { inverse- } \chi^{2}\left(\sigma_{0}^{2}, \omega_{0}\right), \\
\mu_{\theta_{g}} \mid \sigma_{\theta_{g}}^{2} & \sim N\left(\mu_{0}, \sigma_{\theta_{g}}^{2} / \kappa_{0}\right),
\end{aligned}
$$

where $\sigma_{0}^{2}$ and $\omega_{0}$ are the prior scale parameter and degrees of freedom of the inverse chi-square distribution, and $\mu_{0}$ and $\sigma_{\theta_{g}}^{2} / \kappa_{0}$ are the location and scale of the normal distribution. For all models, the following conjugate prior for $\boldsymbol{\lambda}=\left(\boldsymbol{\lambda}^{a}, \boldsymbol{\lambda}^{b}\right)$ was used:

$$
p(\boldsymbol{\lambda}) \sim M V N\left(\boldsymbol{\lambda}_{0}, \boldsymbol{V}_{0}\right),
$$

where $\boldsymbol{\lambda}_{0}$ is the prior mean and $\boldsymbol{V}_{0}$ the prior covariance matrix. The conjugate prior for $\boldsymbol{\Sigma}_{f}$ in (4.3) or $\boldsymbol{\Sigma}$ in (4.4) is the inverse-Wishart distribution with sum of squares $\boldsymbol{S}_{0}$ and degrees of freedom $\nu_{0}$,

$$
\boldsymbol{\Sigma}_{f} \sim \text { inverse-Wishart }\left(\boldsymbol{S}_{0}, \nu_{0}\right) .
$$

\subsubsection{Model Fit Assessment}

To investigate the fit of identical siblings models to the data, posterior predictive checks (PPCs) can be performed. A posterior predictive p-value (PPP) is defined as the probability that the values for a test statistic replicated under the model are more extreme than those observed in the data (Gelman et al., 1996). For example, a PPP for the assessment of family fit can be given as

$$
\mathrm{PPP}=P\left\{T\left(\boldsymbol{u}_{f}^{\mathrm{rep}}, \boldsymbol{\mu}_{f}, \boldsymbol{\theta}\right) \geq T\left(\boldsymbol{u}_{f}, \boldsymbol{\mu}_{f}, \boldsymbol{\theta}\right) \mid \boldsymbol{u}\right\},
$$

where $T\left(\boldsymbol{u}_{f}^{\text {rep }}, \boldsymbol{\mu}_{f}, \boldsymbol{\theta}\right)$ is the replicated value for a test statistic, and $T\left(\boldsymbol{u}_{f}, \boldsymbol{\mu}_{f}, \boldsymbol{\theta}\right)$ is the observed value for the test statistic. Note that the test statistic may depend both on the data and the model parameters, but also only on the data. Using $l=1, \ldots, L$ draws from the posterior, the p-value in $(4.12)$ can be approximated by counting the number of times a replicated value for the test statistic is larger than or equal to the observed value and dividing by $L$. Depending on the test statistic, if a PPP is close to zero or one, the observed data deviate from the expectations under the model regarding the aspect targeted by the statistic.

Two test statistics are used to investigate the assumption of the item parameters of a family being exactly equal to their family value. The first statistic is the variance of the point-biserial correlations for the items per family. It can be used to detect misfit caused by the restricted discrimination parameters. The pointbiserial correlation of an item is indicative of its discriminatory power, and has previously been used in PPCs to detect misfit of the Rasch model (Sinharay et al., 2006). Therefore, a comparison between the observed and replicated variances of the correlations within a family should give information on whether the family discrimination parameters accurately explain the variability observed in the data. Let $\operatorname{Var}_{f}$ represent the variance observed within family $f$. The statistic is given by

$$
T_{f}^{a}=\operatorname{Var}_{f}\left[\frac{\operatorname{av}_{i_{f}}^{+}-\operatorname{av}_{i_{f}}}{\operatorname{sd}_{i_{f}}} \sqrt{\frac{\hat{p}_{i_{f}}}{1-\hat{p}_{i_{f}}}}\right],
$$


where $\mathrm{av}_{i_{f}}^{+}$is the average sum score of the persons who answered item $i_{f}$ correctly, $\mathrm{av}_{i_{f}}$ and $\mathrm{sd}_{i_{f}}$ are the average and standard deviation of the sum scores of all persons who responded to the item, and $\hat{p}_{i_{f}}$ is the proportion correct for the item. In case no one answered an item correctly, or a proportion correct equals one, $T_{f}^{a}$ cannot be computed. Therefore, in such cases, $\mathrm{av}_{i_{f}}^{+}$was given the maximum sum score possible and $\hat{p}$ was set equal to 0.99 , respectively.

The second test statistic is the variance of the item score distributions per family. It can be used to detect misfit caused by the restricted difficulty parameters. The proportion of correct responses to an item is indicative of its difficulty. Therefore, a comparison of the observed and replicated variance of the number of correct responses on items within a family gives information on whether the family difficulty parameters accurately explain the variability observed in the data (Sinharay \& Johnson, 2008). The statistic is given by

$$
T_{f}^{b}=\operatorname{Var}_{f}\left[\sum_{g=1}^{G} \sum_{n_{g}=1}^{N_{g}} u_{i_{f} n_{g}}\right] .
$$

Note that (randomly) missing data is not a problem for the computation of the test statistics; the occurrence of a missing value can simply be copied into the replicated datasets, resulting in a fair comparison. For both test statistics, misfit will lead to observed variances larger than the replicated variances, and the resulting $\mathrm{PPP}$-value will be small.

The fit of the second level of item cloning models can be checked by using an extended posterior predictive check (EPPC) with replicated data for replicated item parameters drawn from their second-level distribution (Gelman et al., 2005; Steinbakk \& Storvik, 2009). Let $\boldsymbol{\xi}_{f}=\left(\boldsymbol{\xi}_{i_{f}}\right)$ be the parameters for the items in family $f$, and $\boldsymbol{\xi}_{f}^{\text {rep }} \sim M V N\left(\boldsymbol{\mu}_{f}, \boldsymbol{\Sigma}_{f}\right)$ the respective replicated parameters. The extended posterior predictive p-value (EPPP) equals

$$
\mathrm{EPPP}=P\left\{T\left(\boldsymbol{u}_{f}^{\mathrm{rep}}, \boldsymbol{\xi}_{f}^{\mathrm{rep}}, \boldsymbol{\mu}_{f}, \boldsymbol{\Sigma}_{f}, \boldsymbol{\theta}\right)<T\left(\boldsymbol{u}_{f}, \boldsymbol{\xi}_{f}, \boldsymbol{\mu}_{f}, \boldsymbol{\Sigma}_{f}, \boldsymbol{\theta}\right) \mid \boldsymbol{u}\right\}
$$

Both the test statistics in (4.13) and (4.14) can be used in the EPPC to check the assumption of a perfect prediction of the family parameters from the effect parameters. In case of misfit, the empirical means of the item parameters per family are not approximated accurately by their respective family parameters, and the estimated covariance matrix will be larger. Therefore, the replicated variances will be larger than the observed variances. To account for smaller values for the EPPP in case of misfit, the inequality sign in (4.15) is reversed relative to the one in (4.12).

An additional assumption of the item cloning models is that of multivariate normally distributed item parameters in the families. The assumption can be tested using EPPCs with the two statistics proposed by Gilbride and Lenk (2010). The first test statistic is based on the result that, if the assumption holds, the squared Mahalanobis distances $\left(\boldsymbol{\xi}_{i_{f}}-\boldsymbol{\mu}_{f}\right)^{\prime} \boldsymbol{\Sigma}_{f}^{-1}\left(\boldsymbol{\xi}_{i_{f}}-\boldsymbol{\mu}_{f}\right)$ follow a chi-square distribution with degrees of freedom equal to the dimension of $\boldsymbol{\Sigma}_{f}$. Using the KolmogorovSmirnov test statistic for the EPPC, it can be assessed whether the replicated data are more similar to the theoretical chi-square distribution than the observed 
data. Gilbride and Lenk (2010) note that the statistic is particularly sensitive to departures from the tails of the multivariate normal distribution.

The second test statistic is based on the observation that, under the assumption of multivariate normality, the scores on the first principle component are normal. Hence, the correlation between the empirical and normal cumulative distribution function of these scores can be used as a test statistic. The statistic is sensitive to multimodal deviations from multivariate normality.

For the first statistic, the sign in (4.15) should be $>=$, whereas for the latter it should be <. Gilbride and Lenk (2010) suggest considering all p-values lower than 0.35 as a conservative indicator of misfit.

In addition, for multilevel models, Gelman and Pardoe (2006) proposed measures of explained variance, $R^{2}$, and pooling, $\lambda$, as summary statistics. (This standard notation for the pooling factor should not be confused with that for the hyperparameters of the models.) Let $\boldsymbol{\zeta}_{f}=\left(\zeta_{i_{f}}\right)$ be either the item discrimination or difficulty parameters for family $f$, and $\mu_{\zeta_{f}}$ be its family mean. The variance explained by the respective family mean parameter can be computed as

$$
R_{\zeta}^{2}=1-\frac{E\left[\operatorname{Var}\left(\zeta_{i_{f}}-\mu_{\zeta_{f}}\right)\right]}{E\left[\operatorname{Var}\left(\zeta_{i_{f}}\right)\right]},
$$

where $E$ represents the mean over the posterior simulations, and Var represents the variance over the parameters. The pooling factor can be computed as

$$
\lambda_{\zeta}=1-\frac{\operatorname{Var}\left[E\left(\zeta_{i_{f}}-\mu_{\zeta_{f}}\right)\right]}{E\left[\operatorname{Var}\left(\zeta_{i_{f}}-\mu_{\zeta_{f}}\right)\right]} .
$$

A pooling factor of less than .5 indicates a higher degree of within-family than between-family information.

\subsubsection{Response Functions}

The results of a calibrated IRT model can be visualized by means of plots of the item response functions (IRFs; van der Linden \& Hambleton, 1997). For the models discussed above, the IRF is given by (4.1). Similarly, for the item families in the multilevel models, a family expected response function (FERF; Lewis, 2001; Sinharay et al., 2003) can be defined as the probability of a correct response to a random item from the family, given ability level. The IRF and FERF are given by

$$
\begin{aligned}
P_{i_{f}}(\theta) & =P\left(U_{i_{f}}=1 \mid \theta, \hat{\boldsymbol{\xi}}_{i_{f}}\right), \\
P_{f}(\theta) & =\int P\left(U_{i_{f}}=1 \mid \theta, \boldsymbol{\xi}_{i_{f}}\right) p\left(\boldsymbol{\xi}_{i_{f}} \mid \hat{\boldsymbol{\mu}}_{f}, \hat{\boldsymbol{\Sigma}}_{f}\right) d \boldsymbol{\xi}_{i_{f}},
\end{aligned}
$$

respectively, where $\hat{\boldsymbol{\xi}}_{i}, \hat{\boldsymbol{\mu}}_{f}$, and $\hat{\boldsymbol{\Sigma}}_{f}$ are expected a posteriori (EAP) estimates. To approximate the integrals, Monte Carlo integration can be used (Lewis, 2001).

\subsection{Empirical Study}

An empirical study was conducted using a dataset with dichotomously scored responses to statistical word problems reported in Zeuch (2011). Each problem 
consisted of an introductory story, with information given in the form of frequencies, followed by one or more questions involving the computation of a probability. The ability intended to be assessed by the problems was that of retrieving the relevant numerical information from the story and applying the relevant formulas to obtain the answer. Hence, the factors defining the formulas were considered as radicals.

The specific radicals used in the generation of the items were selected based on a related study (Holling et al., 2009), in which a larger set of radicals was investigated using the LLTM. More specifically, to solve the items in the study of Zeuch (2011), either the probability of an intersection of independent events (IIE) or a conditional event (which will be denoted as IDE, because it requires knowledge of an intersection of dependent events) had to be computed. In addition, some of the items required the computation of the probability of a complementary event (CE), a set union of disjoint events (SDE), or a combination of these. The radicals involved a multiplication, division, subtraction, or summation of probabilities. More formally, they required application of the following rules:

IIE $P(A \cap B)=P(A) \times P(B)$,

IDE $P(A \mid B)=P(A \cap B) / P(B)$,

CE $P(\neg A)=1-P(A)$,

SDE $P(A \cup B)=P(A)+P(B)$.

In Holling et al.'s (2009) study, each of these radicals increased the difficulty of the items, producing effects significant at the 0.05 level. Unlike the items from Holling et al. (2009), the items from Zeuch (2011) used in the current study were created through the application of both radicals and incidentals, and contained numerical information given as frequencies instead of probabilities.

Eight different families of items were created. A translated version of one item from every family is given in Figure 4.1. (The original items were in German, as the test takers were German high school and vocational school students.)

In total, 14 context stories were constructed in which the combinations of radicals were embedded. Alternatives to the computer game story in Figure 4.1 were, for example, a story about a bike store with bikes of different types, colors, and numbers of gears, and a story about a hotel with rooms with a certain view, price, and location. To avoid any structural differences in the complexity of the context stories, their structure and individual sentences were kept as similar as possible; only the topic of the stories differed. The numerical information in the stories was context-specific. The total number of instances (e.g., 'computer games' in Figure 4.1) was between 100 and 2000, and the information provided in the items was such that the correct solutions were probabilities with a maximum of two digits. The context stories, including the numerical information, will be referred to as the incidentals, as their restricted application was expected not to have a substantial effect on the difficulty of the items, but only to result in surface variability within the item families. The families and context stories were crossed to generate a total of $8 \times 14=112$ items. For more information on the item-construction rules, the reader is referred to Zeuch (2011). 
Grandma Miller would like to buy a computer game for her grandchild because he brought quite good grades home. She is not pleased with all these "violence games" and walks to a store which advertises offering only violence-free games. Because grandma Miller has no knowledge about computer games, she decides to choose a game by chance.

The store offers 500 games altogether.

Of these, 100 games are mainly for beginners, 125 games mainly for advanced players, and the remaining games mainly for skilled persons or for professionals. 50 games are strategy games, 150 games adventures and 300 games jump and run. 300 games are made for Playstation and 200 games are made for PC. 120 games are jump and run and made for Playstation. 120 games are mainly for skilled persons and made for PC. 60 games are mainly for professionals and made for PC.

Level of proficiency and type of hardware are dependent of each other, all other characteristics are independent of each other.

1. What is the probability that grandma Miller selects a game which is mainly for skilled persons, given that this game is made for PC?

2. What is the probability that grandma Miller selects a game which is both mainly for beginners and an adventure?

3. What is the probability that grandma Miller selects a game which is not mainly for professionals, given that this game is made for PC?

4. What is the probability that grandma Miller selects a game which is neither for advanced players nor a jump and run game?

5. What is the probability that grandma Miller selects a game which is either mainly for skilled persons or mainly for professionals, given that this game is made for PC?

6. What is the probability that grandma Miller selects a game which is either mainly for advanced players or both mainly for beginners and a strategy game?

7. What is the probability that grandma Miller selects a game which is not either mainly for skilled persons or mainly for professionals, given that this game is made for $\mathrm{PC}$ ?

8. What is the probability that grandma Miller selects a game which is either not mainly for beginners or both mainly for beginners and a jump and run game?

Figure 4.1: Translated version of one of the context stories with one item from every family. 


$$
\left[\begin{array}{llllllll}
1 & 0 & 0 & 0 & 0 & 0 & 0 & 0 \\
0 & 1 & 0 & 0 & 0 & 0 & 0 & 0 \\
0 & 0 & 1 & 0 & 0 & 0 & 0 & 0 \\
0 & 0 & 0 & 1 & 0 & 0 & 0 & 0 \\
0 & 0 & 0 & 0 & 1 & 0 & 0 & 0 \\
0 & 0 & 0 & 0 & 0 & 1 & 0 & 0 \\
0 & 0 & 0 & 0 & 0 & 0 & 1 & 0 \\
0 & 0 & 0 & 0 & 0 & 0 & 0 & 1
\end{array}\right] \quad\left[\begin{array}{llllllll}
0 & 0 & 0 & 0 & 1 & 0 & 0 & 0 \\
1 & 0 & 0 & 0 & 0 & 0 & 0 & 0 \\
0 & 0 & 0 & 0 & 1 & 1 & 0 & 0 \\
1 & 1 & 0 & 0 & 0 & 0 & 0 & 0 \\
0 & 0 & 0 & 0 & 1 & 0 & 1 & 0 \\
1 & 0 & 1 & 0 & 0 & 0 & 0 & 0 \\
0 & 0 & 0 & 0 & 1 & 1 & 1 & 1 \\
1 & 1 & 1 & 1 & 0 & 0 & 0 & 0
\end{array}\right]
$$

Figure 4.2: Design matrices $\boldsymbol{D}_{1}$ and $\boldsymbol{D}_{1}^{*}$ for the eight item families.

\subsubsection{Dataset}

A total of 741 students participated in the study by Zeuch (2011). The students were from two different populations: high school (HS; $\mathrm{n}=679 ; g=1$ ), and vocational school students (VS; $\mathrm{n}=62 ; g=2$ ). Every test taker responded to 16 items in one of 14 booklets. In each of the booklets every family was represented by two items. The time limit for the test was two minutes per item. A response was scored as correct when both the correct combination of equations and the correct data in the items were used; minor miscalculations were disregarded. The total number of responses per item ranged from 95 to 111 . The data were collected in two phases and were linked at the level of the item families and populations of respondents.

\subsubsection{Design Matrices and Models}

As noted in Section 4.2.1, the different item cloning and identical siblings models are obtained introducing specific assumptions on the within-family item-parameter variability as well as the matrices $\boldsymbol{X}^{a}$ and $\boldsymbol{X}^{b}$ in (4.6) and (4.7). The models used in this study, along with their assumptions about the design matrices $\boldsymbol{X}^{a}$ and $\boldsymbol{X}^{b}$ and covariance matrices $\boldsymbol{\Sigma}$, are listed in Table 4.1. The matrices labeled $\boldsymbol{D}_{1}^{*}$ and $\boldsymbol{D}_{1}-\boldsymbol{D}_{5}$ in the first two columns refer to the matrices depicted in Figures 4.2 and 4.3. As for the entries in the last column, $\boldsymbol{\Sigma}_{f}$ refers to family-specific covariance matrices, $\boldsymbol{\Sigma}$ refers to a common covariance matrix for all families, and $\mathbf{0}$ refers to the case of an identical siblings model (no covariance matrix at all).

All models $M_{1}-M_{3}$ had unrestricted family mean parameters; that is, both $\boldsymbol{X}^{a}$ and $\boldsymbol{X}^{b}$ were equal to the identity matrix $\boldsymbol{D}_{1}$ in Figure 4.2. The models only differed in their assumptions on within-family item-parameter variability: $M_{1}$ had family-specific covariance matrices $\boldsymbol{\Sigma}_{f}$ to capture heterogeneity in the withinfamily variability, $M_{2}$ had a common covariance matrix $\boldsymbol{\Sigma}$ assuming homogeneity across families, and $M_{3}$ had no covariance matrix representing the case of no variability at all.

The next two models, $M_{4}$ and $M_{5}$, were constructed to investigate the prediction of the family mean difficulty parameters by the radical effects. In these models, the definition of $\boldsymbol{X}^{b}$ varied, but all other model features were kept identical to $M_{1}$. As all item families were constructed using either the IIE factor or 


$$
\left[\begin{array}{llllll}
0 & 0 & 0 & 1 & 0 & 0 \\
1 & 0 & 0 & 0 & 0 & 0 \\
0 & 0 & 0 & 1 & 1 & 0 \\
1 & 1 & 0 & 0 & 0 & 0 \\
0 & 0 & 0 & 1 & 0 & 1 \\
1 & 0 & 1 & 0 & 0 & 0 \\
0 & 0 & 0 & 1 & 1 & 1 \\
1 & 1 & 1 & 0 & 0 & 0
\end{array}\right] \quad\left[\begin{array}{llll}
0 & 1 & 0 & 0 \\
1 & 0 & 0 & 0 \\
0 & 1 & 1 & 0 \\
1 & 0 & 1 & 0 \\
0 & 1 & 0 & 1 \\
1 & 0 & 0 & 1 \\
0 & 1 & 1 & 1 \\
1 & 0 & 1 & 1
\end{array}\right] \quad\left[\begin{array}{ll}
0 & 1 \\
1 & 0 \\
0 & 1 \\
1 & 0 \\
0 & 1 \\
1 & 0 \\
0 & 1 \\
1 & 0
\end{array}\right] \quad\left[\begin{array}{l}
1 \\
1 \\
1 \\
1 \\
1 \\
1 \\
1 \\
1
\end{array}\right]
$$

Figure 4.3: Design matrices $\boldsymbol{D}_{2}-\boldsymbol{D}_{5}$ for the eight item families.

Table 4.1: Model labels, design matrices $\boldsymbol{X}^{a}$ and $\boldsymbol{X}^{b}$, and assumptions about the covariance matrices.

\begin{tabular}{llll}
\hline Model & $\boldsymbol{X}^{a}$ & $\boldsymbol{X}^{b}$ & Cov. \\
\hline$M_{1}$ & $\boldsymbol{D}_{1}$ & $\boldsymbol{D}_{1}$ & $\boldsymbol{\Sigma}_{f}$ \\
$M_{2}$ & $\boldsymbol{D}_{1}$ & $\boldsymbol{D}_{1}$ & $\boldsymbol{\Sigma}$ \\
$M_{3}$ & $\boldsymbol{D}_{1}$ & $\boldsymbol{D}_{1}$ & 0 \\
\hline$M_{4}$ & $\boldsymbol{D}_{1}$ & $\boldsymbol{D}_{2}$ & $\boldsymbol{\Sigma}_{f}$ \\
$M_{5}$ & $\boldsymbol{D}_{1}$ & $\boldsymbol{D}_{3}$ & $\boldsymbol{\Sigma}_{f}$ \\
\hline$M_{6}$ & $\boldsymbol{D}_{4}$ & $\boldsymbol{D}_{1}$ & $\boldsymbol{\Sigma}_{f}$ \\
$M_{7}$ & $\boldsymbol{D}_{5}$ & $\boldsymbol{D}_{1}$ & $\boldsymbol{\Sigma}_{f}$ \\
\hline$M_{8}$ & $\boldsymbol{D}_{4}$ & $\boldsymbol{D}_{1}^{*}$ & $\boldsymbol{\Sigma}$ \\
\hline
\end{tabular}

the IDE factor, but not both, we first considered a design matrix in which both types of families were modeled separately; see $\boldsymbol{D}_{2}$ in Figure 4.3. The rows of $\boldsymbol{D}_{2}$ refer to the families, the first three columns are for the IIE factor and the IIE* CE and IIE*SDE interactions, and the last three columns are for the IDE factor and the IDE*CE and IDE*SDE interactions, respectively. The values in the design matrix are equal to one if the respective (combination of) radical(s) was required to generate an item from a particular family, and zero otherwise. $M_{5}$ had only main effects for the four radicals $\left(\boldsymbol{D}_{3}\right.$ in Figure 4.3$)$; i.e., the effects of the CE and SDE radicals on the difficulty of the items were restricted to be equal for the two types of families. The columns of $\boldsymbol{D}_{3}$ are for the IIE, IDE, CE, and SDE factors, respectively.

As the range of the discrimination parameters is typically much smaller than that of the difficulty parameters we considered more simple grouping structures for these parameters instead of the relatively complex linear structures described above for the difficulty parameters. More specifically, models $M_{6}$ and $M_{7}$ were constructed to investigate whether the discriminatory power of (subsets of) the item families could be described by a common discrimination parameter. Again, the definition of $\boldsymbol{X}^{a}$ was varied, whereas all other model features were kept iden- 
tical to $M_{1}$. Model $M_{6}$ contained a discrimination parameter for the IIE and IDE families separately $\left(\boldsymbol{D}_{4}\right)$, whereas $M_{7}$ contained a discrimination parameter common to all families $\left(\boldsymbol{D}_{5}\right.$ in Figure 4.3$)$.

Based on the model fit and summary measures discussed in Section 4.2.3, we selected the most stringent restrictions on the design matrices and the covariance structure of the families that did not result in misfit of their models $M_{2}-M_{7}$. The restrictions were simultaneously applied to our most general model, $M_{1}$, to obtain model $M_{8}$. This model had the following properties: $\boldsymbol{X}^{a}=\boldsymbol{D}_{4}, \boldsymbol{X}^{b}=\boldsymbol{D}_{1}^{*}$, and $\boldsymbol{\Sigma}_{f}=\boldsymbol{\Sigma}$, for all $f$. Note that $\boldsymbol{D}_{1}^{*}$ is a reparameterization of $\boldsymbol{D}_{1}$ with columns for the following main and interaction effects: IIE, IIE*CE, IIE*SDE, IIE* ${ }^{*} E^{*} \mathrm{SDE}$, IDE, IDE*CE, IDE*SDE, and IDE*CE*SDE. Whereas the model with $\boldsymbol{X}^{b}=\boldsymbol{D}_{1}^{*}$ results in the same fit to the data as the same model with $\boldsymbol{X}^{b}=\boldsymbol{D}_{1}$, the former was used in $M_{8}$ to present the estimates for the main and interaction effects for the radicals. For this model, the IRF (4.18) and FERF (4.19) were calculated for all items and families by interpolating between their values at each of the ability points $\boldsymbol{\theta}=-3.0(0.1) 3.0$. The integrals in (4.19) were approximated using 10,000 Monte Carlo draws from the family distributions.

For all models, the prior location $\left(\mu_{0}\right)$ and scale parameter $\left(\sigma_{0}^{2}\right)$ for the ability distribution for the VS students were set to 0 and 1, respectively. Prior parameters $\omega_{0}$ and $\kappa_{0}$ were both set to 1 . The prior means of the effect parameters $\boldsymbol{\lambda}_{0}$ were chosen to result in prior family discrimination and difficulty parameters equal to 1 and 0 , respectively. Furthermore, $\boldsymbol{V}_{0}$ was set equal to a diagonal matrix with elements 100 (representing a case of low prior information). For the multilevel models, the prior sum of squares $\boldsymbol{S}_{0}$ for the covariance matrices were set equal to the expected between family variances of 0.1 and 1.0 for the discrimination and difficulty parameters divided by the number of families, respectively; that is, $\operatorname{Vec}\left(\boldsymbol{S}_{0}\right)=(0.0125,0.0000,0.1250)$. The prior number of degrees of freedom $\nu_{0}$ was set equal to 2. All expected a posteriori estimates, posterior standard deviations, and model fit and summary measures were computed from 40,000 iterations of the Gibbs sampler after 10,000 iterations for burn-in. Convergence of the sampler for the hyperparameters of the models was checked using Geweke's (1992) Z-score statistic for the equality of the means of the first $10 \%$ and last $50 \%$ of the posterior draws after burn in.

\subsubsection{Results}

The comparison between the models will be made in terms of parameter estimates (first section) and model fit according to the (E)PPCs and the measures of explained variance and pooling (second section). The results for the selected model will be described in the third section.

\section{Parameter Estimates}

Table 4.2 gives the EAP estimates of the radical parameters and their posterior standard deviations for the models with a linear structure on the family difficulty (first two columns) or discrimination parameters (last two columns). For the former models, $M_{4}$ and $M_{5}$, the estimate of the intercept for the IIE families was lower relative to that for the IDE families; i.e., items created using the IDE factor 
were more difficult than those created using the IIE factor. In addition to being more difficult, they were also more discriminative of ability (see the results for $\left.M_{6}\right)$. For both types of families, the inclusion of the CE factor lead to a larger increase in difficulty than the inclusion of the SDE factor (compare the interaction effects in $M_{4}$ and the main effects for the CE and SDE factors in $M_{5}$ ).

Within the families, the range of item parameters was relatively small (see Tables 4.3 and 4.4). The estimated common covariance matrix of $M_{2}$ was equal to $\operatorname{Vec}(\boldsymbol{\Sigma})=(0.007,-0.003,0.050)$. Note that the estimated variances were smaller than each of the estimated family-specific variances of model $M_{1}$. We expect this result to be due to our choice of prior, which had a stronger influence on the family-specific matrices (based on 14 items each) than on the common matrix (based on all 112 items). Therefore, the values for the family-specific covariance matrices should be interpreted only relatively; that is, across families or different models with family-specific matrices.

The linear restriction on the family difficulty parameters in $M_{4}$, with both main and interaction effects for the radicals, lead to larger variance estimates for the difficulty parameters of the IIE families relative to those of $M_{1}$ (indicated in bold in Table 4.3). The additional restriction of equal CE and SDE effects for the IIE and IDE families in $M_{5}$ resulted in a large variance estimate for the difficulty parameters in Family 2.

As for the models with restricted family discrimination parameters, the (co)variance estimates of $M_{6}$, with its discrimination parameters for the IIE and IDE families, were quite similar to the respective estimates of the most general model, $M_{1}$ (Table 4.4). The assumption of equal family discrimination parameters $\left(M_{7}\right)$, with an estimated common discrimination parameter of 1.099 and a standard deviation of 0.073 , did lead to larger variance estimates for the discrimination parameters of the families. The increase in variance was particularly large for Family 2, 4, 6, and 7.

The plots in Figures 4.4-4.7 present the item (in grey) and family (in black) parameter estimates for the most general $\left(M_{1}\right)$ and the restricted models $\left(M_{2}-M_{7}\right)$. The different families in the plots are distinguished by different numbers. The item-generation rules led to a large between-families range of discrimination and difficulty parameters (see the item parameter plot for $M_{1}$ in Figure 4.4). Notice that the family parameters lie within their respective clusters of item parameters. This was also the case for the multilevel models $M_{2}, M_{4}$, and $M_{6}$. However, for $M_{5}$ and $M_{7}$ some of the family parameters deviated from their respective item parameter clusters. As can be seen from the plot for the model with the main radical effects $\left(M_{5}\right)$ in Figure 4.6, the family difficulty parameter of Family 2 was larger than each of the item difficulty parameters in that family. This finding explains the large estimate for $\sigma_{b}^{2}$ for Family 2 previously discussed (see Table 4.3). Analogously, the plot for the model with a common family discrimination parameter $\left(M_{7}\right)$ in Figure 4.7 shows that, for some of the families, the discrimination parameter did not lie within their respective cluster of item parameters.

The mean and variance estimates of the ability distributions for the VS students are given in Table 4.5. With a decreasing number of parameters to describe the family mean parameters, the estimates of the mean of the ability distributions increased and the variance estimates decreased (compare, for example, the results 
Table 4.2: Radical estimates (and standard deviations) for the models with a design matrix for the family difficulty $\left(M_{4}\right.$ and $\left.M_{5}\right)$ or discrimination parameters $\left(M_{6}\right.$ and $\left.M_{7}\right)$.

\begin{tabular}{lrrrr}
\hline & $M_{4} \lambda^{b}$ & $M_{5}$ & $M_{6} \lambda^{a}$ & $M_{7}$ \\
\hline Intercept & - & - & - & $1.099(0.073)$ \\
\hline IIE & $-1.110(0.223)$ & $-0.655(0.124)$ & $0.767(0.041)$ & - \\
IIE*CE & $0.809(0.239)$ & - & - & - \\
IIE*SDE & $0.787(0.247)$ & - & - & - \\
\hline IDE & $0.857(0.106)$ & $0.829(0.096)$ & $1.503(0.078)$ & - \\
IDE*CE & $0.518(0.112)$ & - & - & - \\
IDE*SDE & $0.434(0.117)$ & - & - & - \\
\hline CE & - & $0.555(0.084)$ & - & - \\
\hline SDE & - & $0.454(0.092)$ & - & - \\
\hline
\end{tabular}

Table 4.3: Family-specific covariance matrices for models $M_{1}, M_{4}$, and $M_{5}$.

\begin{tabular}{rrrr|rrr|rrrr}
\hline \multicolumn{9}{c}{$M_{1}$} & \multicolumn{5}{c}{$M_{4}$} \\
$\mathrm{f}$ & $\sigma_{a}^{2}$ & $\sigma_{a b}$ & $\sigma_{b}^{2}$ & $\sigma_{a}^{2}$ & $\sigma_{a b}$ & $\sigma_{b}^{2}$ & $\sigma_{a}^{2}$ & $\sigma_{a b}$ & $\sigma_{b}^{2}$ \\
\hline 1 & 0.023 & 0.004 & 0.066 & 0.028 & 0.005 & 0.071 & 0.026 & 0.004 & 0.066 \\
2 & 0.009 & -0.002 & 0.070 & 0.012 & -0.012 & $\mathbf{0 . 1 9 4}$ & 0.026 & -0.045 & $\mathbf{0 . 6 8 9}$ \\
3 & 0.018 & 0.007 & 0.074 & 0.018 & 0.006 & 0.075 & 0.017 & 0.005 & 0.070 \\
4 & 0.010 & 0.001 & 0.079 & 0.014 & 0.007 & $\mathbf{0 . 2 5 4}$ & 0.011 & 0.003 & 0.101 \\
5 & 0.020 & 0.012 & 0.112 & 0.018 & 0.010 & 0.120 & 0.019 & 0.011 & 0.117 \\
6 & 0.018 & -0.007 & 0.065 & 0.023 & -0.014 & $\mathbf{0 . 1 4 8}$ & 0.020 & -0.007 & 0.076 \\
7 & 0.016 & 0.002 & 0.058 & 0.018 & 0.004 & 0.074 & 0.017 & 0.004 & 0.075 \\
8 & 0.018 & -0.003 & 0.062 & 0.021 & -0.000 & $\mathbf{0 . 1 3 9}$ & 0.018 & -0.003 & 0.068 \\
\hline
\end{tabular}

Table 4.4: Family-specific covariance matrices for models $M_{6}$ and $M_{7}$.

\begin{tabular}{rrrr|rrr}
\hline \multicolumn{4}{c}{$M_{6}$} & \multicolumn{3}{c}{$M_{7}$} \\
\hline $\mathrm{f}$ & $\sigma_{a}^{2}$ & $\sigma_{a b}$ & $\sigma_{b}^{2}$ & $\sigma_{a}^{2}$ & $\sigma_{a b}$ & $\sigma_{b}^{2}$ \\
\hline 1 & 0.022 & 0.002 & 0.067 & 0.050 & -0.000 & 0.061 \\
2 & 0.015 & -0.012 & 0.084 & $\mathbf{0 . 1 4 8}$ & -0.145 & $\mathbf{0 . 2 3 4}$ \\
3 & 0.018 & 0.008 & 0.076 & 0.027 & 0.010 & 0.068 \\
4 & 0.010 & 0.002 & 0.079 & $\mathbf{0 . 0 8 7}$ & 0.003 & 0.098 \\
5 & 0.020 & 0.013 & 0.116 & 0.022 & 0.009 & 0.092 \\
6 & 0.017 & -0.006 & 0.064 & $\mathbf{0 . 0 7 5}$ & -0.030 & 0.075 \\
7 & 0.017 & 0.001 & 0.054 & $\mathbf{0 . 0 8 7}$ & 0.013 & 0.053 \\
8 & 0.019 & -0.003 & 0.063 & 0.052 & -0.006 & 0.065 \\
\hline
\end{tabular}


$M_{1}$
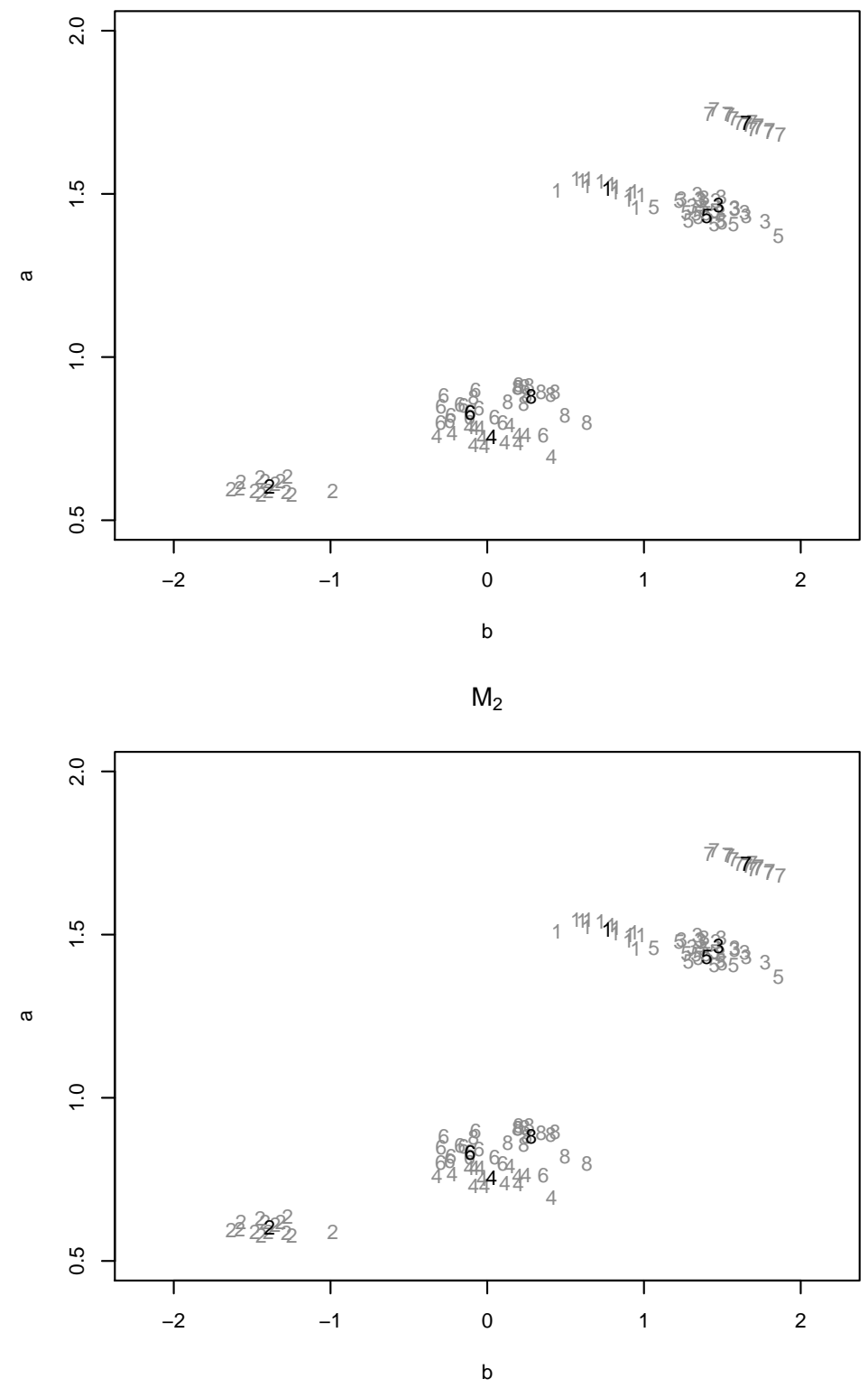

Figure 4.4: Item (grey) and family (black) difficulty (x-axis) and discrimination (y-axis) parameter estimates for models $M_{1}$ and $M_{2}$. 
$M_{3}$
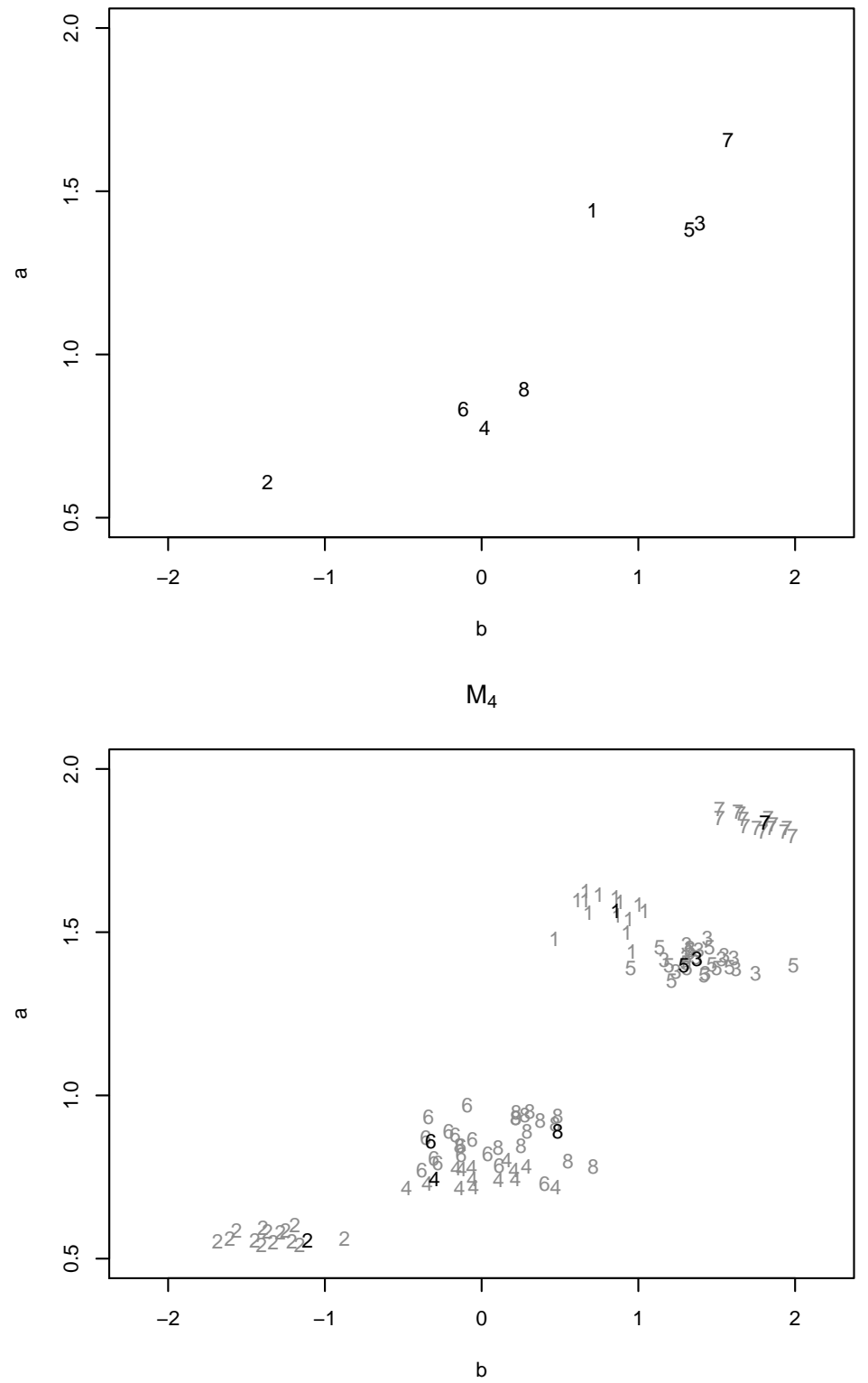

Figure 4.5: Item (grey) and family (black) difficulty (x-axis) and discrimination (y-axis) parameter estimates for models $M_{3}$ and $M_{4}$. 
$M_{5}$
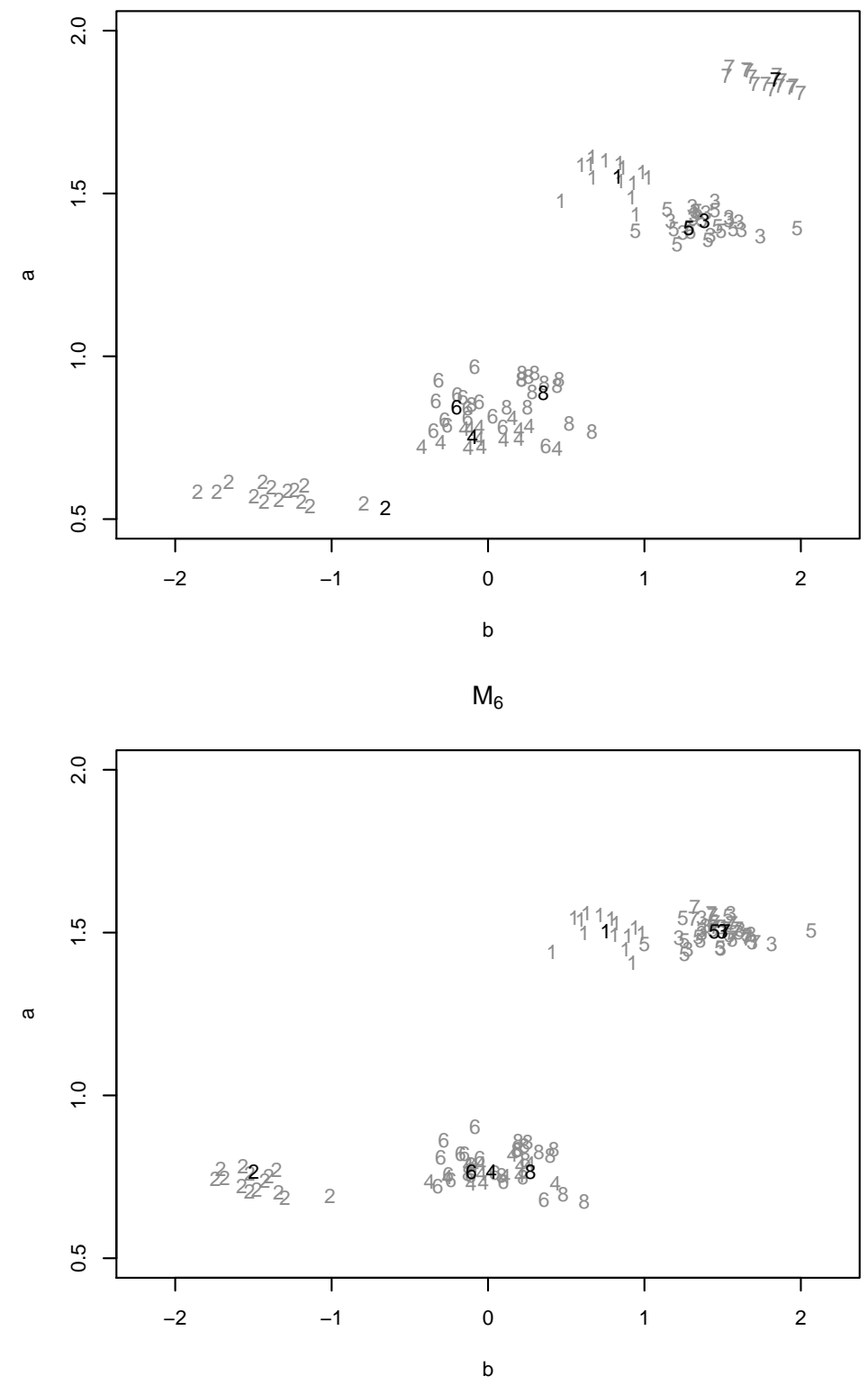

Figure 4.6: Item (grey) and family (black) difficulty (x-axis) and discrimination (y-axis) parameter estimates for models $M_{5}$ and $M_{6}$. 
$M_{7}$
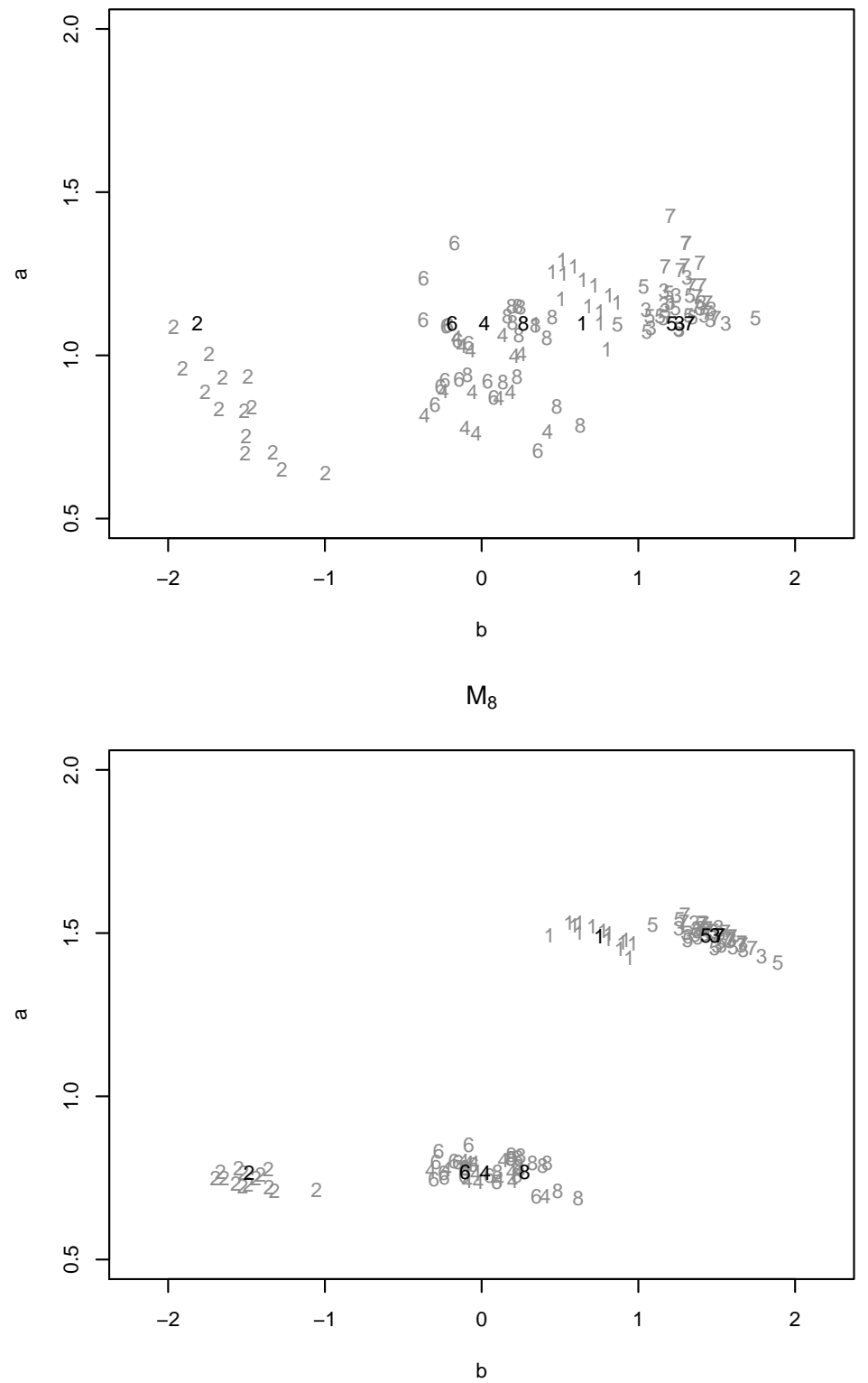

Figure 4.7: Item (grey) and family (black) difficulty (x-axis) and discrimination (y-axis) parameter estimates for models $M_{7}$ and $M_{8}$. 
Table 4.5: Mean (and standard deviations) and variance estimates for the ability distributions for $g=2$.

\begin{tabular}{lrr}
\hline Model & $\mu_{\theta}$ & $\sigma_{\theta}^{2}$ \\
\hline$M_{1}$ & $-0.848(0.195)$ & 1.570 \\
$M_{2}$ & $-0.838(0.193)$ & 1.542 \\
$M_{3}$ & $-0.893(0.194)$ & 1.568 \\
\hline$M_{4}$ & $-0.834(0.195)$ & 1.558 \\
$M_{5}$ & $-0.830(0.192)$ & 1.534 \\
\hline$M_{6}$ & $-0.809(0.180)$ & 1.425 \\
$M_{7}$ & $-0.785(0.165)$ & 1.182 \\
\hline
\end{tabular}

for models $M_{1}, M_{6}$, and $M_{7}$ ).

\section{Model Fit}

The results for the model fit analyses are presented in Tables 4.6-4.7. Table 4.6 is for the models without restrictions on the family mean parameters, but with varying restrictions on the covariance structure of their families. Both for $M_{1}$ (family-specific covariance matrices) and $M_{2}$ (common covariance matrix for all families), the value for the $T^{b}$ statistic was relatively low for Family 2. We expect this result to be due to one item with a relatively large difficulty parameter compared to the other items of this family (see Figure 4.4). In general though, the values for $T^{a}$ and $T^{b}$ did not indicate any major misfit of the family distributions assumed by these models. However, ignoring the within-family variance in the item parameters, as in $M_{3}$, led to misfit both for the discrimination and the difficulty parameters (see the last two columns of Table 4.6; misfit is indicated in bold).

The fit results for the models with a linear restriction on the family difficulty parameters are presented in the first four columns of Table 4.7. Based on the values for the $T^{a}$ and $T^{b}$ statistics, both the model with main and interaction effects of the radicals explaining family difficulty $\left(M_{4}\right)$ and the model with main effects for the radicals only $\left(M_{5}\right)$ resulted in misfit for the second family.

The last four columns of Table 4.7 give the results for the models with a restriction on the family discrimination parameters. The restriction did not have any impact on the fit of model $M_{6}$; the IIE and IDE radical effects seemed sufficient to explain the discriminatory power of the families. However, the restriction of equal family discrimination parameters in $M_{7}$ led to misfit for Family 7 (see the last two columns of Table 4.7).

Except for a value of 0.328 for the Kolmogorov-Smirnov statistic for Family 8 in $M_{4}$ and a value of 0.159 for the same statistic for Family 2 in $M_{5}$, all values for the two statistics for the check on the multivariate normality assumption were larger than .35. Hence, the assumption was not rejected for the models with these values.

Table 4.8 gives the values for the explained variance $R^{2}$ and pooling factor 
Table 4.6: Results of fit analyses for the models with varying covariance structure $\left(M_{1}-M_{3}\right)$.

\begin{tabular}{lrr|rr|rr}
\hline & \multicolumn{2}{c}{$M_{1}$} & \multicolumn{2}{c}{$M_{2}$} & \multicolumn{2}{c}{$M_{3}$} \\
\hline $\mathrm{f}$ & $T^{a}$ & $T^{b}$ & $T^{a}$ & $T^{b}$ & $T^{a}$ & $T^{b}$ \\
\hline 1 & 0.594 & 0.549 & 0.674 & 0.586 & 0.279 & 0.271 \\
2 & 0.717 & 0.224 & 0.788 & 0.295 & $\mathbf{0 . 0 9 2}$ & 0.108 \\
3 & 0.514 & 0.567 & 0.549 & 0.614 & 0.360 & 0.338 \\
4 & 0.631 & 0.658 & 0.688 & 0.866 & 0.157 & $\mathbf{0 . 0 0 0}$ \\
5 & 0.609 & 0.698 & 0.680 & 0.863 & 0.217 & $\mathbf{0 . 0 5 1}$ \\
6 & 0.766 & 0.617 & 0.876 & 0.721 & $\mathbf{0 . 0 5 3}$ & $\mathbf{0 . 0 0 1}$ \\
7 & 0.587 & 0.676 & 0.598 & 0.671 & 0.349 & 0.371 \\
8 & 0.833 & 0.697 & 0.925 & 0.782 & $\mathbf{0 . 0 2 1}$ & $\mathbf{0 . 0 0 1}$ \\
\hline
\end{tabular}

Table 4.7: Results of fit analyses for the models with a restriction on the family difficulty $\left(M_{4}\right.$ and $\left.M_{5}\right)$ or discrimination parameters $\left(M_{6}\right.$ and $\left.M_{7}\right)$.

\begin{tabular}{rrr|rr|rr|rr}
\hline & \multicolumn{2}{c}{$M_{4}$} & \multicolumn{2}{c}{$M_{5}$} & \multicolumn{2}{c}{$M_{6}$} & \multicolumn{2}{c}{$M_{7}$} \\
\hline $\mathrm{f}$ & $T^{a}$ & $T^{b}$ & $T^{a}$ & $T^{b}$ & $T^{a}$ & $T^{b}$ & $T^{a}$ & $T^{b}$ \\
\hline 1 & 0.566 & 0.599 & 0.565 & 0.590 & 0.602 & 0.547 & 0.234 & 0.496 \\
2 & 0.630 & $\mathbf{0 . 0 8 6}$ & 0.401 & $\mathbf{0 . 0 0 0}$ & 0.762 & 0.284 & 0.552 & 0.382 \\
3 & 0.554 & 0.569 & 0.543 & 0.578 & 0.537 & 0.573 & 0.350 & 0.601 \\
4 & 0.480 & 0.318 & 0.605 & 0.552 & 0.655 & 0.663 & 0.501 & 0.708 \\
5 & 0.655 & 0.637 & 0.653 & 0.628 & 0.646 & 0.702 & 0.472 & 0.714 \\
6 & 0.703 & 0.425 & 0.756 & 0.575 & 0.706 & 0.599 & 0.689 & 0.668 \\
7 & 0.553 & 0.721 & 0.544 & 0.710 & 0.487 & 0.701 & $\mathbf{0 . 1 9 6}$ & 0.707 \\
8 & 0.707 & 0.517 & 0.795 & 0.680 & 0.708 & 0.665 & 0.811 & 0.729 \\
\hline
\end{tabular}

Table 4.8: Summary statistics $\left(R^{2}\right.$ and $\left.\lambda\right)$ for the multilevel models.

\begin{tabular}{lrrrr}
\hline Model & $R_{a}^{2}$ & $\lambda_{a}$ & $R_{b}^{2}$ & $\lambda_{b}$ \\
\hline$M_{1}$ & 0.921 & 0.889 & 0.941 & 0.449 \\
$M_{2}$ & 0.960 & 0.894 & 0.951 & 0.433 \\
\hline$M_{4}$ & 0.918 & 0.893 & $\mathbf{0 . 8 8 7}$ & $\mathbf{0 . 4 0 3}$ \\
$M_{5}$ & 0.917 & 0.884 & $\mathbf{0 . 8 6 9}$ & $\mathbf{0 . 2 2 6}$ \\
\hline$M_{6}$ & 0.901 & 0.868 & 0.940 & 0.447 \\
$M_{7}$ & - & $\mathbf{0 . 5 3 1}$ & 0.917 & 0.466 \\
\hline
\end{tabular}


$\lambda$ for the multilevel models. For all models, the explained variance in both the discrimination and difficulty parameters was large $(>.8)$. Also, there was a higher degree of between-family than within-family information for the discrimination parameters, whereas the reverse was true for the difficulty parameters. In particular, for $M_{4}$ and especially $M_{5}$, which both showed misfit in the difficulty parameter of Family 2, the explained variance and pooling factor were lower compared to the other models. Similarly, the pooling factor of the discrimination parameters was lower for $M_{7}$, which had already shown misfit in the discrimination parameters for some of the families. The differences between the amount to which the item parameters are pooled towards their family means for these models and $M_{1}$ is also evident from their plots in Figures 4.4-4.7.

\section{Model Selection}

All results for the family-specific covariance matrices, model fit statistics, and explained variance and pooling factors converge as to which restrictions led to misfit. The most stringent restrictions that did not result in misfit were those corresponding to homogeneity of within-family item-parameter variability (i.e., $\boldsymbol{\Sigma}_{f}=\boldsymbol{\Sigma}$, for all $f$ ) and family discrimination prediction by the IIE and IDE factors (i.e., $\boldsymbol{X}^{a}=\boldsymbol{D}_{4}$ ). These restrictions were combined in our final model, $M_{8}$. For the description of family difficulty the full model with radical main and interaction effects was used (i.e., $\boldsymbol{X}^{b}=\boldsymbol{D}_{1}^{*}$ ).

The difficulty and discrimination factor estimates for this model are given in the first and second column of Table 4.9, respectively. The estimates were similar to those for the models with separate restrictions on the difficulty and discrimination parameters $\left(M_{4}\right.$ and $\left.M_{6}\right)$. Note, however, the smaller standard deviations for the estimates of the radical parameters in $M_{8}$ because of its smaller number of model parameters. The estimated common covariance matrix for the families was $\operatorname{Vec}(\boldsymbol{\Sigma})=(0.008,-0.004,0.050)$, a result similar to that for the previous model with a common covariance matrix, $M_{2}$. The estimate (and standard deviation) for the mean of the ability distribution for the VS students was -0.801 (0.178) and the variance 1.403 .

The lower plot in Figure 4.7 shows the item/family parameter plot for this new model. Similar to $M_{1}$, its family parameter values were located within the appropriate item-parameter clusters.

The results for fit measures $T^{a}$ and $T^{b}$ applied to $M_{8}$ are presented in Table 4.10. The combination of restrictions in this model did not visibly deteriorate its fit. Also, the values for the two statistics for the check on the multivariate normality assumption (not reported here) were larger than .35, and did not indicate any major misfit. Furthermore, the values for the explained variance measure and pooling factor were close to the ones obtained for the other fitting models $\left(R_{a}^{2}=0.944, \lambda_{a}=0.860, R_{b}^{2}=0.951\right.$, and $\left.\lambda_{b}=0.432\right)$.

Figure 4.8 shows the IRFs and FERF per family. Our finding that the items with the IDE factor (uneven families) were both more difficult and more discriminating than the items with the IIE factor (even families) is also evident from these plots (compare the plots in the left-hand and right-hand columns). Note also that the families for which no misfit was found in the identical siblings model (Families 

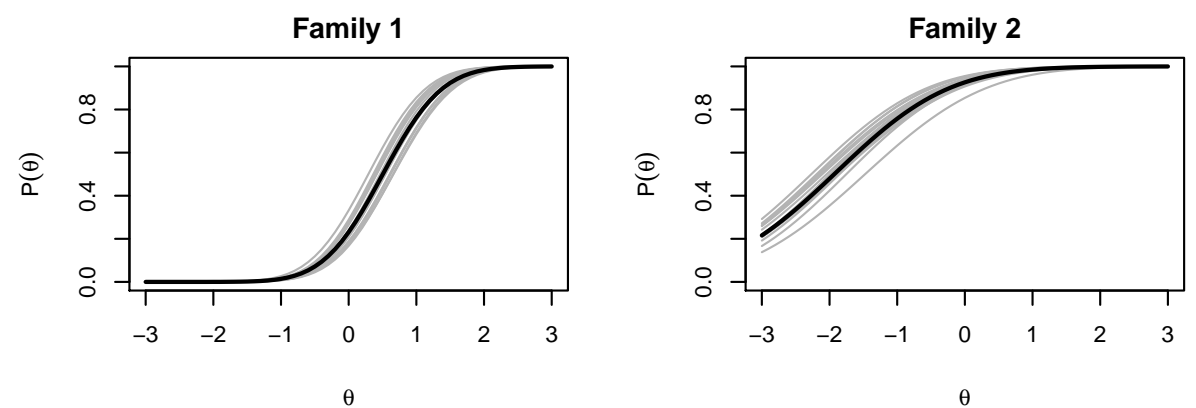

Family 3

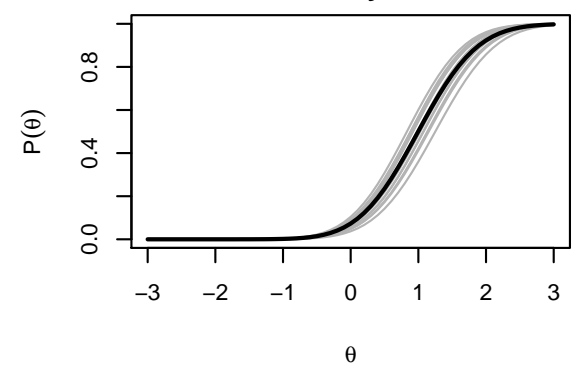

Family 5

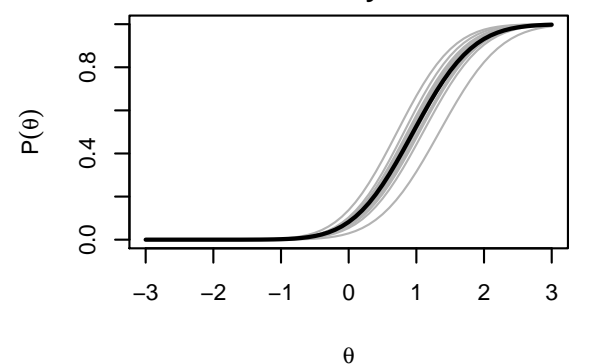

Family 7
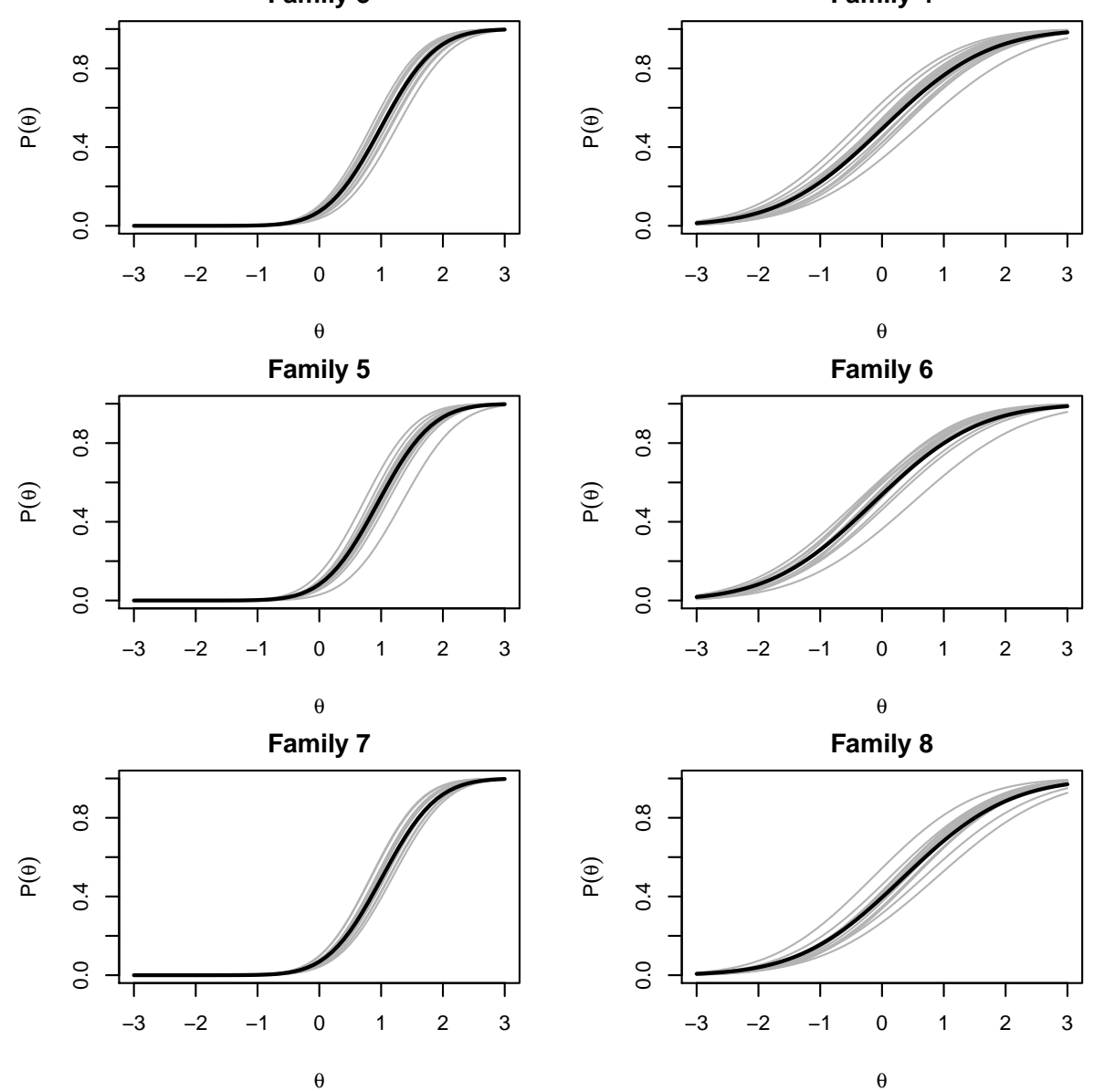

$\theta$

Family 6

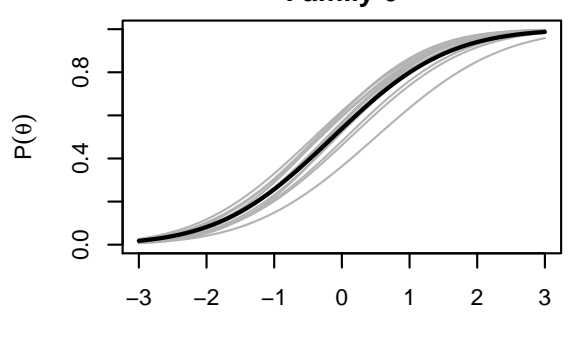

Family 8

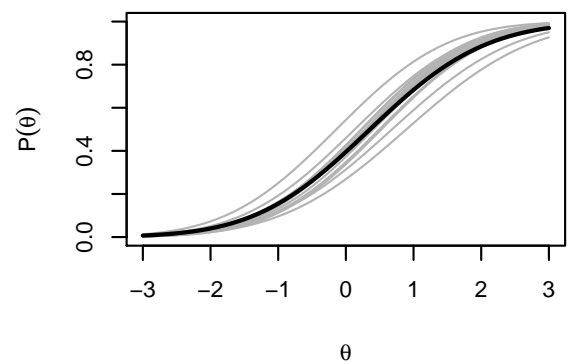

Figure 4.8: Item (grey) and family expected response curves (black) for model $M_{8}$. 
Table 4.9: Radical estimates (and standard deviations) for $M_{8}$.

\begin{tabular}{lrr}
\hline & \multicolumn{1}{c}{$\boldsymbol{\lambda}^{b}$} & \multicolumn{1}{c}{$\boldsymbol{\lambda}^{a}$} \\
\hline IIE & $-1.483(0.084)$ & $0.767(0.039)$ \\
IIE*CE & $1.506(0.105)$ & - \\
IIE*SDE $^{*}$ & $1.378(0.106)$ & - \\
IIE*CE*SDE & $-1.126(0.143)$ & - \\
\hline IDE & $0.753(0.097)$ & $1.492(0.072)$ \\
IDE*CE & $0.736(0.112)$ & - \\
IDE*SDE & $0.678(0.111)$ & - \\
IDE*CE*SDE $^{*}$ & $-0.649(0.161)$ & - \\
\hline
\end{tabular}

Table 4.10: Results of fit analyses for $M_{8}$.

\begin{tabular}{ccc}
\hline $\mathrm{f}$ & $T^{a}$ & $T^{b}$ \\
\hline 1 & 0.667 & 0.591 \\
2 & 0.831 & 0.384 \\
3 & 0.555 & 0.603 \\
4 & 0.696 & 0.872 \\
5 & 0.712 & 0.857 \\
6 & 0.825 & 0.697 \\
7 & 0.494 & 0.691 \\
8 & 0.844 & 0.756 \\
\hline
\end{tabular}

1, 3, and 7; see the model fit results for $M_{3}$ in Table 4.6) showed less variability in the response curves for their items as compared to the other families.

\subsection{Discussion}

Models were discussed that can be used to analyze data from clusters ('families') of items, generated by combinations of design features ('radicals'). The models were estimated in a Bayesian framework with MCMC sampling from their posterior distributions.

In the empirical study, the estimates of the family-specific covariance matrices were largely influenced by the prior. Therefore, it was investigated whether the within-family item-parameter variability could be assumed to be homogeneous over families. If so, all items contribute to the estimation of a common covariance matrix. Note that with an increasingly smaller number of items per family a formal statistical test for the assumption of equal covariance matrices will have less power, and the restricted model is more likely to be favored over the more complex model with family-specific covariance matrices-a clear manifestation of 
the model complexity-model fit tradeoff. However, in case the assumption does not hold, an alternative was provided by Griffiths and Valenzuela (2006) in the form of a hierarchical prior for the covariance matrices. This type of prior allows the family-specific covariance matrices to borrow strength from their higher-level average. However, in our study, the hypothesis of equal covariance matrices across families was not rejected by any of the model fit measures. But, ignoring the within-family item-parameter variability altogether did result in bias in the item parameter estimates as well as misfit of the model.

An imperfect prediction of the family parameters in the item cloning model with linear restrictions resulted in error that was absorbed by the family-specific covariance matrices. When this happened, the covariance matrices not only described the random variation caused by the incidentals, but also the residual variance in the family parameters. As a result, some of the family parameters did not lie within their group of item parameters. Visual inspection of the location of the family parameters in combination with model fit statistics can be used to identify the source of misfit, as well as to select the best set of radicals to explain the family parameters. An alternative, not further pursued here, is to extend the model with error terms for the residual variance in the family parameters. The two sources of error-incidental variation and residual variance in the family parameters-are then separated. However, the added complexity of such a model has the disadvantage of a relatively large number of families needed to accurately estimate the error terms related to the residual variance. Likewise, the number of items and persons in the calibration sample for the model can become prohibitively large.

Our final model, with a common covariance matrix explaining the within-family item-parameter variability as well as radicals explaining the family parameters, seems a likely candidate for the description of the data. The calibrated model has the advantage of new items generated using combinations of the radicals not requiring any calibration. Ability estimation for the model could be based on the parameter values for the family distributions. 


\title{
Chapter 5
}

\section{Optimal Test Design With Rule-Based Item Generation}

\begin{abstract}
Rule-based item generation is a method to automatically generate test items based on construction rules which are meant to determine the items' difficulty. Optimal test-design methods are applied to rule based-item generation. The methods maximize expected Fisher information in the test while satisfying several constraints on the features of the automatically generated items. Optimization models for three different cases of automated test design are presented. In one of the cases, the parameters of the individual items have been estimated. For the other two cases, it is not necessary to have calibrated the test items but it is sufficient to estimate families of items with distributions of item parameters using a hierarchical item response theory model for rule-based item generation. Results from a simulation study show both the effects of the item parameter variability in the families and the severity of the constraint sets in the test design models on the test information measure.
\end{abstract}

Key words: Fisher information, hierarchical modeling, item response theory, optimal test design, rule-based item generation.

\subsection{Introduction}

The field of educational and psychological assessment methods has shown a clear trend towards automation lately, for instance, in the form of advances in automated item generation (Irvine \& Kyllonen, 2002), computerized adaptive testing (van der Linden \& Glas, 2010), and automated scoring and feedback technology (Xi, 2010). As for automated item generation, if the cognitive processes involved in problem solving are known, rules can be defined to generate test items that elicit the processes (Irvine \& Kyllonen, 2002). In principle, once the rules are available, their use opens up the possibility of real-time automated item generation during test taking. In addition to its potential cost-effectiveness in frequent

Adapted from: Geerlings, H., van der Linden, W. J., \& Glas, C. A. W. (2011). Optimal test design with rule-based item generation. Submitted for publication. 
large-scale assessments, rule-based item generation furthers content validity of the assessments through its use of explicit design matrices for the items. One of the goals of this article is to use optimal test-design methods to generate design matrices that optimize the statistical objective of measurement accuracy while at the same time satisfying a range of constraints on the content of the test. Given the objective and content constraints, a system that combines rule-based item generation with such optimal test-design methods has the potential to fully automate test generation.

However, for the feasibility of this goal, several issues need to be resolved. For instance, the psychometric properties of rule-based generated items are not necessarily a priori known. A solution to this issue is possible when the rules lead to families of parallel items. Assuming approximately normally distributed item parameters within the families, hierarchical item response theory (IRT) models can then be used to calibrate the families using only a sample of their items. The calibration of the families exists of estimating their mean item parameters along with their (co)variances (Glas \& van der Linden, 2003; Geerlings et al., 2011; Chapter 2). Once this has been done, any new item in a family generated by the same rules does not need to be calibrated; instead, its known family distribution can be used to assemble a test and score the test takers.

The fact that the parameters of the individual items remain unknown up to a given probability distribution requires an adaptation of the current methods for optimal test design and test scoring. For the case of adaptive testing with item families but without item-generation rules, these issues were already addressed in Glas and van der Linden (2003). They used a Bayesian approach with a minimum expected posterior variance criterion to select the items along with an expected a posteriori (EAP) estimator to update the ability estimate after each item. Both choices accounted for the remaining uncertainty about the item parameters through integration over their family distributions.

The main focus of the current research was on test design with rule-based item families. Its primary goal was to adjust the objective of maximization of Fisher's information on the ability parameter to the case of item families, as well as to explore how to use the adjusted criterion in an optimization model appropriate for automated generation of a test from these families.

The second goal was to assess the degree to which the remaining uncertainty about the individual item parameters affects the solutions to optimal test-design models. Previous research on the effect of item parameter uncertainty on optimal test assembly focused exclusively on estimation error due to small calibration samples (Hambleton, Jones, \& Rogers, 1993; van der Linden \& Glas, 2000). The effects of this error can be described as capitalization-on-chance effects: Small calibration samples lead to larger positive errors of the discrimination parameters of some of the items, and hence to their likely selection in the test. The result is a lower than nominal test information function and underestimation of the standard error of the ability estimates (Tsutakawa \& Johnson, 1990; Zhang, Xie, Song, \& $\mathrm{Lu}, 2011$ ). The magnitude of the capitalization-on-chance effect not only depends on the size of the calibration sample but also on the variability of the true item parameters (van der Linden \& Glas, 2001) and the item bank size-test length ratio (Hambleton et al., 1993; Hambleton \& Jones, 1994). However, in real-world ap- 
plications, the effect of these factors is mitigated by the content constraints added to the test-assembly problem (Hambleton et al., 1993). In the current context of optimal test assembly from items with parameter distributions, the opposite of a capitalization-on-chance effect can be expected: It is no longer possible to select maximum-information items directly from the pool, because we select only from the families with the highest mean information. The actual items are distributed around these means. Therefore, test-information functions obtained for family selection will be lower than those for selection of individually calibrated items from the same pool.

In the next sections, rule-based item generation and a model for the calibration of item families and the effects of item generation rules is reviewed. Then, optimal test design with remaining uncertainty in the item parameters for three cases of item generation is discussed: (a) test assembly from an item pool generated and calibrated prior to testing, (b) test generation on the fly from calibrated item families, and (c) test generation on the fly using calibrated item-generation rules only. Simulation studies will be presented to show both the effects of different amounts of remaining uncertainty in the item parameters and the presence of content constraints on test information. The article will be concluded with a discussion of a basic tradeoff in the design of rule-based item generation.

\subsection{Rule-Based Item Generation}

An important distinction is between item-generation rules that influence the difficulty of the items and rules that have negligible effects. The former have been called radicals (Irvine, 2002); they are typically identified using a cognitive analysis of the item domain. Their systematic use can help to ensure the content validity of the items. The latter are known as incidentals; they only create surface variation and do not have any systematic effect on item difficulty (Irvine, 2002). Hence, we will treat them as random effects. Their application, often referred to as 'item cloning', allows items to be generated with similar psychometric properties, but with a different 'look'. When test security is an issue, the use of incidentals allows for the generation of seemingly different but psychometrically nearly equivalent items for different test takers.

Examples of investigations into the effect of radicals on the difficulty of items includes research on algebra word problems (Lane, 1991; Enright, Morley, \& Sheehan, 2002; Arendasy, Sommer, Gittler, \& Hergovich, 2006; Arendasy \& Sommer, 2007), statistical word problems (Holling et al., 2008, 2009), figural matrices (Hornke \& Habon, 1986; Freund et al., 2008; Klein Entink, Kuhn, Hornke, \& Fox, 2009), latin square tasks (Zeuch, 2011), and reading comprehension items (Gorin, 2005; Sonnleitner, 2008). Examples of investigations into the variability in item parameters caused by incidentals include Glas et al.'s (2010) analysis of naval architecture items, and Sinharay et al.'s (2003) study of mathematics items.

Even though the separate advantages of rule-based item generation and item cloning have been recognized, examples of their combined use in automated item generation are not yet common. One example is the Java-based item generator called Generator for Narrative Probability Exercises (Genpex) developed by Boer Rookhuiszen (2011; see also Theune et al., 2011). The generator is based on the 
research on the radicals, incidentals, and prototypical items for statistical word problems by Zeuch (2011). Each of the prototypes consisted of some numerical information embedded in a context story, followed by questions requiring the computation of a probability (for an example, see Zeuch, 2011; Chapter 4, Figure 4.1). The radicals were defined as the type of computations required to answer the questions (complementary probability; probability of an intersection of independent events; conditional probability; probability of a union of disjoint events) and the incidentals as the numerical information and the topics of the context stories. The Genpex generator additionally includes linguistic variations, such as removal of duplicate words from aggregated sentences, changes in word order and changes in ordering of the sentences. The prototype items were field tested using students in German high schools and vocational colleges as subjects. An analysis using various constrained versions of the hierarchical IRT model for cloned items by Glas and van der Linden (2003) showed that the difficulty of the item families could be explained as main and interaction effects of the radicals, whereas for the explanation of the discriminatory power of the families only main effects were necessary (Chapter 4). Also, relative to the between-family item-parameter variability, the variation between the item parameter values within the families was small.

\subsection{Modeling Rule-Based Item Generation}

The joint use of radicals and incidentals in item generation results in families of items, with between-family variation caused by the radicals and within-family variation by the incidentals. The hierarchical structure of items grouped into families can be explicitly taken into account through multilevel response modeling. Let $n=1, \ldots, N$ be the persons, $f=1, \ldots, F$ the item families, and $i_{f}=1, \ldots, I_{f}$ the items from family $f$. Furthermore, let $U_{i_{f} n}$ be the dichotomous variable for the response of person $n$ to item $i_{f}$. The model for this case proposed by Glas and van der Linden (2003), henceforth denoted as the item cloning model (ICM), extends the three-parameter logistic (3PL) model with a hierarchical structure on the item parameters for each family. In this article, however, the three-parameter normal-ogive (3PNO) model is used as first-level model:

$$
p\left(U_{i_{f} n}=1 \mid \theta_{n}, a_{i_{f}}, b_{i_{f}}, \gamma_{i_{f}}\right)=\gamma_{i_{f}}+\left(1-\gamma_{i_{f}}\right) \Phi\left(a_{i_{f}}\left[\theta_{n}-b_{i_{f}}\right]\right),
$$

where $a_{i_{f}}, b_{i_{f}}$, and $\gamma_{i_{f}}$ are the discrimination, difficulty, and guessing parameters for item $i$ in family $f$, respectively, $\theta_{n}$ is the person parameter, and $\Phi($.$) is the cu-$ mulative normal distribution function. The two-parameter normal-ogive (2PNO) model arises as the first-level model when $\gamma_{i_{f}}=0$ for $i_{f}=1, \ldots, I_{F}$. Generally, the choice of the normal-ogive instead of the logistic link function has the advantage of easy implementation of the Gibbs sampler for the ICM extended with a linear structure on the family difficulty parameters used in the empirical examples below.

The item parameters are transformed as

$$
\boldsymbol{\xi}_{i_{f}}=\left(a_{i_{f}}, b_{i_{f}}, \operatorname{logit} \gamma_{i_{f}}\right),
$$

to facilitate the assumption of a multivariate normal distribution as second-level 
model:

$$
\boldsymbol{\xi}_{i_{f}} \sim M V N\left(\boldsymbol{\mu}_{f}, \boldsymbol{\Sigma}_{f}\right)
$$

with $\boldsymbol{\mu}_{f}$ and $\boldsymbol{\Sigma}_{f}$ family parameters representing the vector of the means of the item parameters for family $f$ and their variability about these means, respectively. We will use $c_{i_{f}}$ to denote the transformed guessing parameter. If the covariance matrices $\boldsymbol{\Sigma}_{f}$ are approximately equal, the assumption of a common covariance matrix $\boldsymbol{\Sigma}$ instead of family-specific matrices is convenient. This case may arise, for example, when all families are generated by the same set of incidentals, and there exists no interaction between the effects of the radicals and the incidentals.

A linear structure on the mean difficulty parameters $\mu_{b_{f}}, f=1, \ldots, F$, can be used to model the fact that family membership is determined by the radicals (Geerlings et al., 2011; Chapter 2). Let $r=1, \ldots, R$ be the radicals used to generate the families. The proposed linear structure is

$$
\mu_{b_{f}}=\sum_{r=1}^{R} d_{f r} \beta_{r},
$$

where $\beta_{r}$ denotes the effect of radical $r$ on the mean family difficulty $\mu_{b_{f}}$ and $d_{f r}$ is a design variable denoting how often radical $r$ should be used to construct an item from family $f$. In the literature, the $F \times R$ design matrix $\boldsymbol{D}=\left(\left(d_{f r}\right)\right)$ has also been referred to as a Q-matrix (Fischer, 1973; Tatsuoka, 1983). Thus, (5.4) represents the mean family difficulty as a weighted sum of the effects of the radicals used to generate items from it.

The basic model restricted by (5.4) will be labeled the linear item cloning model (LICM). Furthermore, the model with family-specific covariance matrices will be labeled the LICM-F, whereas the model with a common covariance matrix is referred to as the LICM-C. A Bayesian estimation procedure to estimate the parameters of the models is presented in Appendix A. Geerlings et al. (2011; Chapter 2) present a parameter recovery study showing the precision with which the parameters are estimated with specific numbers of families, items per family, and persons responding to an item. Bayesian methods to investigate the fit of the models to data were discussed in Chapter 4.

\subsection{Three Cases of Automated Test Design}

Our approach to automated test design is based on mixed integer programming (MIP) optimization with objective functions and constraints (for a general introduction, see van der Linden, 2005). Three different cases are distinguished. The first is assembling a test from a pre-generated item pool. In this case, the itemgeneration rules have already been used to generate all the items in the pool, and the individual item parameters have been estimated using the LICM. Content constraints derived from the radicals and incidentals that define the items in the families can be imposed on the selection of the items.

Second, the test can be designed using knowledge both about the item generation rules and the parameter distribution of each of the families, whereupon the optimal design is used to generate the items in the test. The parameters of 
the newly generated items can be treated as randomly sampled from their family distributions, which have been estimated using representative samples of persons and items. The main difference with the previous case is that the target will be a family information function to represent the use of random incidentals to generate the items from the selected families. The specific family information function used is introduced below. We refer to this case of test design as fully automated test generation on the fly. It has the advantage that, in principle, every test taker can be given a unique test. Potential test security problems due to overexposure of the items are therefore avoided. Note however, that family overexposure may still be an issue. The odds of family disclosure are dependent on the choice of the incidentals; that is, the variability of the surface features of the items they create within the families.

Third, for some applications, it may be possible to go one step further and use the item-generation rules to sample items from entirely new families. This is possible when the effect parameters for the radicals in (5.4) have been estimated along with a common covariance matrix $\boldsymbol{\Sigma}$ for all families, together with additional assumptions about the family means for the discrimination and guessing parameters, $\mu_{a_{f}}$ and $\mu_{c_{f}}$. In this case of fully automated, rule-based test generation, the mean difficulties of the families, $\mu_{b_{f}}$, required for the family information function in the optimal test design model are calculated directly from (5.4). Relative to the previous case, this advanced form of automated test assembly can help to reduce family overexposure.

In the next sections, the family information function is introduced and the three cases of test design are discussed in more detail. For each case, the type of MIP model that can be used to implement it in a fully automated fashion is discussed.

\subsubsection{Family Information Function}

For the first case of automated test design the item parameters are known, and item information can be defined as

$$
I_{i_{f}}(\theta)=-\mathrm{E}_{u}\left[\frac{\partial^{2}}{\partial \theta^{2}} \ln p\left(u \mid \theta, \boldsymbol{\xi}_{i_{f}}\right)\right]
$$

(see, for example, van der Linden \& Pashley, 2010, section 1.2.1).

For the two cases of item generation on the fly, the parameters of the individual items are unknown but the parameters $\boldsymbol{\mu}_{f}$ and $\boldsymbol{\Sigma}_{f}$ have been estimated. The objective function for the test-design problem should therefore be based on a family information function, which is defined as the expected information in the response to a random item from family $f$. The function can be computed by integrating (5.5) over the item parameters; that is, as

$$
I_{f}(\theta)=-\mathrm{E}_{u}\left[\frac{\partial^{2}}{\partial \theta^{2}} \ln \int p\left(u \mid \theta, \boldsymbol{\xi}_{i_{f}}\right) p\left(\boldsymbol{\xi}_{i_{f}} \mid \boldsymbol{\mu}_{f}, \boldsymbol{\Sigma}_{f}\right) d \boldsymbol{\xi}_{i_{f}}\right] .
$$

A convenient approximation of the integral is by Monte Carlo integration. Details of the approximation are given in Appendix B.2. Besides, Appendix B.2 offers a small simulation study investigating the number of iterations needed to obtain a 
required precision. The properties of the family information function are further explored in a later section.

\subsubsection{Test Assembly from Pre-Generated Item Pools}

Assembling a test from a pre-generated item pool with families of items with calibrated parameters $\boldsymbol{\xi}_{f}=\left(\boldsymbol{\xi}_{i_{f}}\right)$ can be seen as a series of decisions of whether or not family $f$ should be represented in the test by including item $i_{f}$. The problem can be formalized using 0-1 decision variables for all families and items in the pool, $\boldsymbol{x}=\left(x_{1}, \ldots, x_{f}, \ldots, x_{F}, x_{1_{1}}, \ldots, x_{i_{f}}, \ldots, x_{I_{F}}\right)$, defined as

$$
\begin{aligned}
x_{f} & = \begin{cases}1, & \text { family } f \text { is selected } \\
0, & \text { otherwise. }\end{cases} \\
x_{i_{f}} & = \begin{cases}1, & \text { item } i \text { from family } f \text { is selected; } \\
0, & \text { otherwise }\end{cases}
\end{aligned}
$$

The decision variables are used to formulate the objective function and constraints in the optimization model presented below. The objective function maximizes the total information in the test subject to a set of weights $R_{p}$ at $\theta_{p}$, $p=1, \ldots, P$, that represent the relative shape of the target for the information function. The constraints represent all content and other specifications for the test. For the case of assembly from a pre-generated item pool, two types of constraints can be imposed. First, the rules used to generate items from a particular family are stored in the $F \times R$ design matrix $\boldsymbol{D}$ introduced above. For the example of statistical word problems presented above, design variable $d_{f r}$ could be an indicator of whether or not probability formula $r$ needs to be applied to solve an item from family $f$. As demonstrated below, these design variables are the item attributes in the first type of constraint. Second, the use of incidentals in the generation of items is assumed to be indicated in a separate design matrix $\boldsymbol{T}=\left(\left(t_{i_{f}}\right)\right)$, with $c=1, \ldots, C$ denoting the available incidentals. Again, as an example, for the generation of statistical word problems, design variable $t_{i_{f} c}$ could be an indicator variable for the context story used to generate the problem for item $i_{f}$.

Selecting $l$ families and $k$ items from every selected family, subject to the constraints relative to the design matrices $\boldsymbol{D}$ and $\boldsymbol{T}$, amounts to solving the following optimization problem:

$$
\text { Maximize } y \quad \text { (maximum information) }
$$

subject to

$$
\begin{array}{ll}
\sum_{f=1}^{F} \sum_{i_{f}=1}^{I_{f}} I_{i_{f}}\left(\theta_{p}\right) x_{i_{f}} \geq R_{p} y, \quad p=1, \ldots, P, & \text { (relative shape of target) } \\
\sum_{f=1}^{F} x_{f}=l, & \text { (number of families) }
\end{array}
$$




$$
\begin{aligned}
& -k x_{f}+\sum_{i_{f}=1}^{I_{f}} x_{i_{f}}=0, \quad f=1, \ldots, F, \quad \text { (number of items per family) } \\
& \sum_{f=1}^{F} d_{f r} x_{f} \gtreqless b_{r}, \quad r=1, \ldots, R, \quad \text { (radicals) } \\
& \sum_{f=1}^{F} \sum_{i_{f}=1}^{I_{f}} t_{i_{f} c} x_{i_{f}} \gtreqless b_{c}, \quad c=1, \ldots, C, \quad \text { (incidentals) } \\
& x_{f}=(0,1), \quad f=1, \ldots, F, \quad \text { (variables for families) } \\
& x_{i_{f}}=(0,1), \quad i_{f}=1_{1}, \ldots, I_{f}, \ldots, I_{F}, \quad \text { (variables for items) } \\
& y=(0, \infty), \quad \text { (auxiliary variable) }
\end{aligned}
$$

with $I_{i_{f}}\left(\theta_{p}\right)$ the information provided by item $i_{f}$ at ability value $\theta_{p}(5.5)$. The objective is of the maximin type: real-valued variable $y$, which is maximized in (5.7), is a common factor in the lower bounds to the sum of the item information functions in (5.8) (for details, see van der Linden, 2005, section 5.1.4). Observe that $R_{p}=1.0$ for $p=1, \ldots, P$ represents the case of the approximation of a uniform target for the test information function. The equalities in (5.9) and (5.10) constrain the number of families and items per family to be selected in the test. The latter also ensures that items from a family are selected if and only if the family is selected. (If $x_{f}=0$, the equation only holds if $x_{i_{f}}=0$ for all $i_{f}=1, \ldots, I_{f}$, but if $x_{f}=1$, it holds only if the sum of all $x_{i_{f}}$ equals $k$.) The constraints in (5.11) and (5.12) impose the bounds $b_{r}$ and $b_{c}$ on the number of times a radical and incidental is represented in the test, respectively.

The model only represents the core of an actual optimization model for test assembly from a pre-generated pool of items. In a real-world application, several other types of constraints may have to be added; for examples, see van der Linden (2005). A solution to the optimization problem in (5.7)-(5.15) is a string of values for the $0-1$ decision variables that maximizes its objective function. It can be found using a solver of the branch-and-bound type (Williams, 1993, section 6.2). In the later examples, we used the mixed-integer solver in CPLEX 9.0 (ILOG, 2003).

\subsubsection{Test Generation On The Fly}

For the second case, the problem changes from the assembly of a test from a pregenerated item pool to the one of designing a test; that is, identifying the rules to generate the items for the test. Or, more formally, to identify the sets of admissible design matrices $\{\boldsymbol{D}\}$ that serve as the blueprint for the generation of the items. Of course, the goal is to find the design matrix with the most informative combination. When it is identified the items are generated by (i) applying the radicals according to the optimal design $\boldsymbol{D}$ and (ii) random sampling from the incidentals.

Hence, the item parameters are unknown, but the known family distributions can be used to optimally design the test. The general model for this type of generation on the fly contains decision variables for the families only, and the 
selection of families is based on the family information measure $I_{f}(\theta)$ in (5.6):

$$
\text { Maximize } y \quad \text { (maximum information) }
$$

subject to

$$
\begin{array}{lll}
\sum_{f=1}^{F} I_{f}\left(\theta_{p}\right) x_{f} \geq R_{p} y, & p=1, \ldots, P, & \text { (relative shape of target) } \\
\sum_{f=1}^{F} x_{f}=l, & & \text { (number of families) } \\
\sum_{f=1}^{F} d_{f r} x_{f} \gtreqless b_{r}, & r=1, \ldots, R, & \text { (radicals) } \\
x_{f}=(0,1), & f=1, \ldots, F, & \text { (variables for families) } \\
y=(0, \infty) . & & \text { (auxiliary variable) }
\end{array}
$$

Again, the model is for the selection of $l$ families [see (5.18)] that together provide optimal information with respect to the relative target in (5.16) and (5.17), and that satisfy the content constraints based on the radicals in (5.19). However, the incidentals are assumed to be randomly sampled to satisfy the assumption of a family information function.

\subsubsection{Test Generation On The Fly Using Radicals Only}

In the third case, even more flexibility in item generation is obtained if the new items are not required to come from fully pre-calibrated families. This is possible if (a) the effect parameters for the radicals, $\beta_{r}, r=1, \ldots, R$, have been estimated from an earlier sample of families and items required to calculate the means of the family difficulties, $\mu_{b_{f}}$, from (5.4), (b) the mean of the family discrimination and guessing parameters, $\mu_{a_{f}}$ and $\mu_{c_{f}}$, can be assumed to be approximately equal across families, and their common means have been estimated from earlier samples, and (c) the same has been done with a common covariance matrix $\boldsymbol{\Sigma}$ for the families. As an alternative to the second assumption, a model as in (5.4) could be used to predict the mean of the family discrimination and guessing parameters as a linear combination of radical effects.

This time, the decision variables $x_{f}$ are redefined as admissible combinations of radicals $v$ :

$$
x_{v}= \begin{cases}1, & \text { combination } v \text { of radicals is selected } \\ 0, & \text { otherwise. }\end{cases}
$$

The test-design model in (5.16)-(5.21) can now be used to generate items from families for admissible combinations of radicals that have never been realized before.

Observe the large number of items that can be generated from small sets of radicals and incidentals. As an example, assume that the items can be generated using $R$ radicals and $C$ incidentals, each of them is a dichotomy, and all possible 


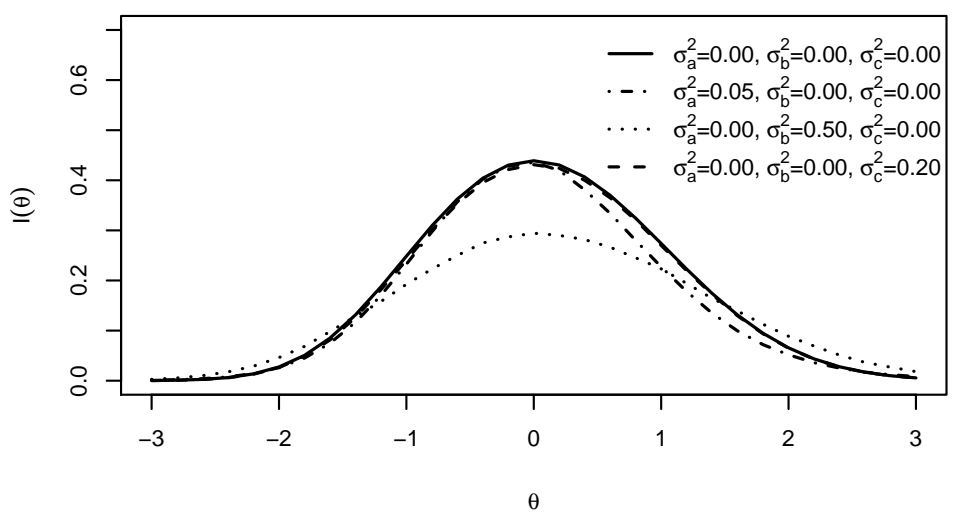

Figure 5.1: Family information for the $3 \mathrm{PNO}$ as the first-level model as a function of the amount of within-family variance in the item parameters and ability level. The family mean parameters were fixed at $\mu_{a}=1, \mu_{b}=-0.258$, and $\mu_{\gamma}=0.2$, and the correlation between the item parameters was fixed at zero.

combinations of radicals and incidentals are admissible. The number of possible families is then equal to $2^{R}$ and the total number of possible items $2^{R+C}$. Thus, from five radicals and ten incidentals 32,768 items in 32 families can be generated. If the set of radicals is extended with another five, the numbers are 1,048,576 and 1,024, respectively. For non-dichotomous radicals and incidentals, the numbers are even higher.

These numbers illustrate the potential power of on-the-fly test generation. Sets of incidentals of substantial size are easily found. For example, in the item generator for statistical word problems discussed in the introductory section, both the content of the context story, the numerical information, the order of the sentences, and the exact wording of the sentences can be varied. The combination of these incidentals leads to an almost infinite number of items that can be generated.

\subsection{Effect of the Covariance Matrix on Family In- formation}

The variability of the item parameters within a family has a potentially large impact on the value of the family information function in (5.6). We discuss the effects for the 3PNO model as first-level model. Family discrimination was fixed at one, family guessing at 0.2 (this corresponds to a $\mu_{c}$ of $\operatorname{logit}(0.2)$ ), and family difficulty was chosen to be optimal with respect to $\theta=0$; that is, $\mu_{b}=-0.258$ (see Wolfe, 1981). The variances were either 0.00 or $0.05\left(\sigma_{a}^{2}\right), 0.00$ or $0.50\left(\sigma_{b}^{2}\right)$, and 0.00 or $0.20\left(\sigma_{c}^{2}\right)$. Note that the nonzero values are large, but their relative magnitudes are according to their usual realization. The covariances were chosen to correspond to a correlation $\rho$ of $0.0,0.5$, or -0.5 . Family information was computed for the different family covariance matrices at the 31 ability points $\boldsymbol{\theta}=-3.0(0.2) 3.0$. The 

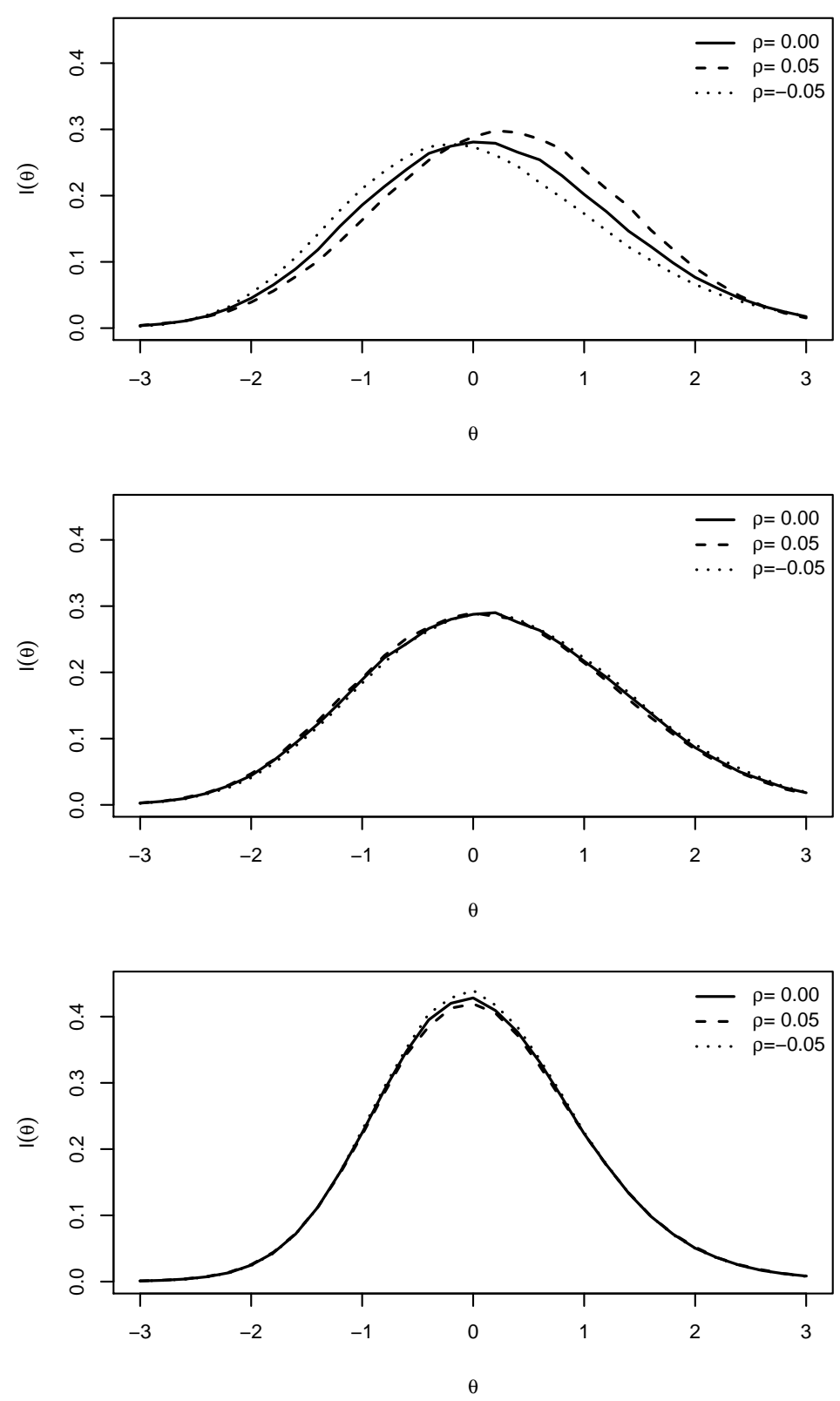

Figure 5.2: Family information for the 3PNO as the first-level model as a function of the correlation between the item parameters and ability level. The family mean parameters were fixed at $\mu_{a}=1, \mu_{b}=-0.258$, and $\mu_{\gamma}=0.2$. For the upper plot the variances were $\operatorname{diag}(\boldsymbol{\Sigma})=(0.05,0.50,0.00)$, for the middle plot $\operatorname{diag}(\boldsymbol{\Sigma})=(0.00,0.50,0.20)$, and for the lower plot $\operatorname{diag}(\boldsymbol{\Sigma})=(0.05,0.00,0.20)$. 
curves in Figures 5.1 and 5.2 were obtained by interpolating between the values. The integrals over the item parameters in (5.6) were approximated using 10,000 Monte Carlo draws from the family distributions (see Appendix B.2). Negative values for the discrimination or guessing parameters were disregarded.

Figure 5.1 presents the curves for the case of zero correlations, and zero variance (solid line) or variance in only one type of parameter (dashed and dotted lines). An increase in the variance in the difficulty parameters $\sigma_{b}^{2}$ led to a decrease in the family information over a large range of the ability scale. However, an increase in the variance of the discrimination parameters $\sigma_{a}^{2}$ led to a decrease in family information only at the ability levels away from the optimal difficulty value. In general, the effects of an increase in the variance of the guessing parameters were small.

The first plot of Figure 5.2 is for the case of $\operatorname{diag}(\boldsymbol{\Sigma})=(0.05,0.50,0.00)$ and varying correlation between the item discrimination and difficulty parameters. A positive correlation resulted in an increase in information at $\theta>\mu_{b}$ but a decrease at $\theta<\mu_{b}$ (compare the curves for zero and 0.5 correlation in the upper plot in Figure 5.2). This can be explained as follows: In case of a positive correlation, larger discrimination parameters coincide with larger difficulty parameters. As the former is the main determinant of the family information function, the positive correlation introduces a shift of the function to the right. For a negative correlation, the reverse shift can be observed.

The second plot of Figure 5.2 is for the case of $\operatorname{diag}(\boldsymbol{\Sigma})=(0.00,0.50,0.20)$ and varying correlation between the item difficulty and guessing parameters. A positive correlation resulted in a small shift of the family information function to the left; negative correlation in a shift to the right. These shifts are explained by the fact that larger guessing parameters result in less information. However, the effects were very small, and cannot easily be discerned in the figure.

The third plot of Figure 5.2 is for the case of $\operatorname{diag}(\boldsymbol{\Sigma})=(0.05,0.00,0.20)$ and varying correlation between the item discrimination and guessing parameters. A positive correlation has a counterbalancing effect on the family information function (more information for larger discrimination parameters but less information because of larger guessing parameters). On the other hand, negative correlation means larger discrimination for lower guessing parameters, and thus generally higher values for the family information function than when there is no covariance between them.

Note that when all variances are zero (the condition corresponding to the solid line in Figure 5.1), all item parameters are equal to their respective means, and family information equals item information in (5.5) with the family values substituted for the item parameters. In case of non-zero (co)variances, approximating family information in this manner, thereby ignoring the uncertainty in the item parameters, almost always resulted in overestimation of the amount of information in the response to a random item from the family.

\subsection{Simulation Study}

As indicated in the introduction, calibration of every individual item is unnecessary if tests are assembled based on family information. However, uncertainty about the 
Table 5.1: Simulated family parameters.

\begin{tabular}{rrrrrrrr}
\hline$f$ & $\mu_{a}$ & $\mu_{b}$ & $\mu_{\gamma}$ & $f$ & $\mu_{a}$ & $\mu_{b}$ & $\mu_{\gamma}$ \\
\hline 1 & 1.01 & -2.0 & 0.20 & 17 & 0.90 & -1.0 & 0.10 \\
2 & 1.58 & -0.8 & 0.13 & 18 & 1.23 & 0.2 & 0.13 \\
3 & 1.36 & -1.4 & 0.20 & 19 & 1.69 & -0.4 & 0.14 \\
4 & 0.94 & -0.2 & 0.16 & 20 & 1.55 & 0.8 & 0.13 \\
5 & 0.88 & -1.1 & 0.20 & 21 & 1.29 & -0.1 & 0.10 \\
6 & 0.83 & 0.1 & 0.11 & 22 & 0.98 & 1.1 & 0.18 \\
7 & 1.62 & -0.5 & 0.17 & 23 & 1.49 & 0.5 & 0.13 \\
8 & 1.18 & 0.7 & 0.19 & 24 & 1.63 & 1.7 & 0.18 \\
9 & 1.67 & -1.7 & 0.10 & 25 & 0.96 & -0.7 & 0.20 \\
10 & 1.41 & -0.5 & 0.19 & 26 & 1.59 & 0.5 & 0.16 \\
11 & 1.36 & -1.1 & 0.18 & 27 & 1.14 & -0.1 & 0.10 \\
12 & 1.30 & 0.1 & 0.10 & 28 & 0.98 & 1.1 & 0.18 \\
13 & 1.39 & -0.8 & 0.12 & 29 & 1.40 & 0.2 & 0.13 \\
14 & 1.14 & 0.4 & 0.16 & 30 & 1.23 & 1.4 & 0.19 \\
15 & 1.59 & -0.2 & 0.14 & 31 & 0.93 & 0.8 & 0.20 \\
16 & 1.67 & 1.0 & 0.11 & 32 & 1.43 & 2.0 & 0.19 \\
\hline
\end{tabular}

individual item parameters in a family leads to less information provided by the assembled test relative to the case of known parameters. A study was conducted to assess the loss of information. At the same time, the study illustrates how to use the test-assembly model in (5.7)-(5.15).

\subsubsection{Study Setup}

A total of 32 item families was created through the use of five dichotomous radicals with a fully crossed design. The mean difficulties of the families were computed from (5.4), with $\boldsymbol{\beta}=(-2.0,1.0,0.3,0.9,0.6,1.2)$. The first value represented an intercept. The next five values represented the regression coefficients for the five radicals. The discrimination parameters for the families were sampled from 0.8(0.01)1.7. Analogously, the mean guessing parameters were sampled from $0.1(0.01) 0.2$, and subsequently transformed into the logits in (5.2). All family means, which are given in Table 5.1, were kept fixed across the replications. The covariance matrix of the family mean parameters was used as a measure of between-family variation. The within-family covariance matrices $\boldsymbol{\Sigma}$ were assumed to be equal across families (LICM-C). Four within-family covariance matrices were used; they were chosen equal to $0.01,0.05,0.1$, or 0.2 times the between-family matrix. In each of 100 replications, 10 or 20 items were sampled from the family distributions in (5.3).

Item information in (5.5) was calculated from the simulated item parameters at the three ability values $\theta=-1,0$, and 1 . Besides, family information in (5.6) was calculated at the same ability values using Monte Carlo integration with 10,000 
iterations.

Two different test-assembly problems were formulated: one without and one with constraints on the distribution of the radicals in the test. For both models, the general formulation in (5.7)-(5.15) was used. To make the comparison between the cases of selection based on calibrated items and selection based on calibrated family distributions, the models were used twice, once with (5.8) for the case of item selection, and once with (5.17) for the case of family selection. As there were no constraints on the distribution of the incidentals in the test, i.e. the constraints in (5.12) were omitted, the latter case corresponds to random sampling of the items from the selected families.

The baseline model (M1) did not contain the constraints on the radicals in (5.11). Its objective function and the first set of constraints represented a relative target for the test information function. That is, the objective was to maximize the height of the information function while maintaining its relative shape defined by the weights $R_{p}, p=1,2,3$, at $\theta_{p}=-1,0,1$. The weights were set equal to $(1,1,1)$ (uniform relative target) or $(1,2,1)$ (relative target with twice as much information at $\theta=0$ ). The number of families selected, $l$, was equal to 10 or 20 , while the number of items selected from each family was fixed at one. Thus, the length of the test was also equal to $l$.

Ten constraints on the frequency of the radicals in the test (5.11) were added to the second model (M2). Test length $l$ was equal to 10 or 20 . Each of the five radicals was restricted to occur between 5-6 and 10-12 times in the test, respectively.

The test-assembly problems were solved using CPLEX 9.0 (ILOG, 2003). For the sets of items selected using (5.8), the mean values of the objective function were computed from (5.5). For the case of family selection using (5.17), the mean values of the objective function were computed from (5.6) and from (5.5) using the simulated item parameters for the (randomly) selected items. These choices allow us to compare the 'expected' mean values of the objective function based on knowledge only of the families, and the 'observed' mean values, based on the simulated item parameters.

\subsubsection{Results}

Figures 5.3 and 5.4 show the results for the two different targets for the information function. The mean values of the objective function $(\bar{y})$ for the cases of item (Item Sel.) and family selection (Fam. Sel.) are given as a function of the number of items per family $\left(I_{f}\right)$, number of selected items/families $(l)$, and within-between (W-B) ratio of the item parameter variability for the models M1 and M2. For the case of family selection, both the expected (Fam. Sel. Exp.) and observed mean value (Fam. Sel. Obs.) of the objective function are given.

A comparison between item and family selection shows that, with increasing variance in the item parameters per family, unlike the latter, the information for a test selected using item information increased. This can be explained as follows: Increasing within-family variance means increasing variance in the information in its items, from which the most informative ones are selected. However, family information decreases because of the larger uncertainty about the item parameter 
values - an effect already discussed in the previous section. Besides, a comparison between the expected and observed values of the objective function for the case of family selection showed minimal differences for small within-family variance, whereas the observed mean was sometimes larger than the expected value for larger within-family variances. In other words, the results for the family-information measure tended to be somewhat conservative for the larger within-family variances.

As expected, the addition of the extra constraints on the distribution of the radicals in M2 caused a decrease in the expected test information, both for item and family selection. However, the effect was more pronounced for the latter.

Doubling the number of items per family, $I_{f}$, led to a slight increase in the test information functions based on the individual items, but the family test information functions remained approximately the same. This effect of the item bank size-test length ratio is in agreement with the results obtained by Hambleton et al. (1993) in their study of the effect of capitalization on chance in item selection. When twice the number of families was selected, the absolute difference between the minimum family and item test information increased (notice the different scales for the respective plots). However, the shape of the plots remained approximately the same.

All results for the two targets for the test information functions were comparable. In general, for the uniform target, larger values of the objective function were obtained both for family and item selection.

Figures 5.5 (uniform target at $\theta=\{-1,0,1\}$ ) and 5.6 (target with twice as much information at $\theta=0$ ) show the effect of increasing within-family variance on the test information for item and family selection (2PNO as first-level model; i.e., $\left.\boldsymbol{\mu}_{\gamma}=0\right)$. For these figures, the number of families selected was $l=10$, the number of items per family was equal to $I_{f}=10$, and the selection model was M1. The plots in their left-hand columns show the item and family parameters in the pool, with the selected items and families highlighted in black. The plots in the righthand columns show the corresponding test-information curves for item and family selection. The effect of increasing within-family variance in the item parameters can be seen by comparing plots in different rows. The item parameters for the top rows had a within-between covariance ratio of 0.01 ; for the lower rows, the ratios were $0.05,0.10$, and 0.20 , respectively. Family test information decreased with increasing variance in the item parameters, whereas the results for the iteminformation functions tended to approximate the target for the test better.

\subsection{Discussion}

Rule-based item generation can be used to generate families of items in which the between-family variance is caused by item features with a fixed effect on the item parameters but within-family variance is caused by features that only have a random effect. In this article, the case of optimal test assembly from such families was studied. The methods proposed can be used to automatically generate tests directly from a set of test specifications.

In practical applications, a decision has to be made regarding the trade-off between the advantages of small item-parameter variability within families and larger surface variability between their items. For high-stakes testing, we may 

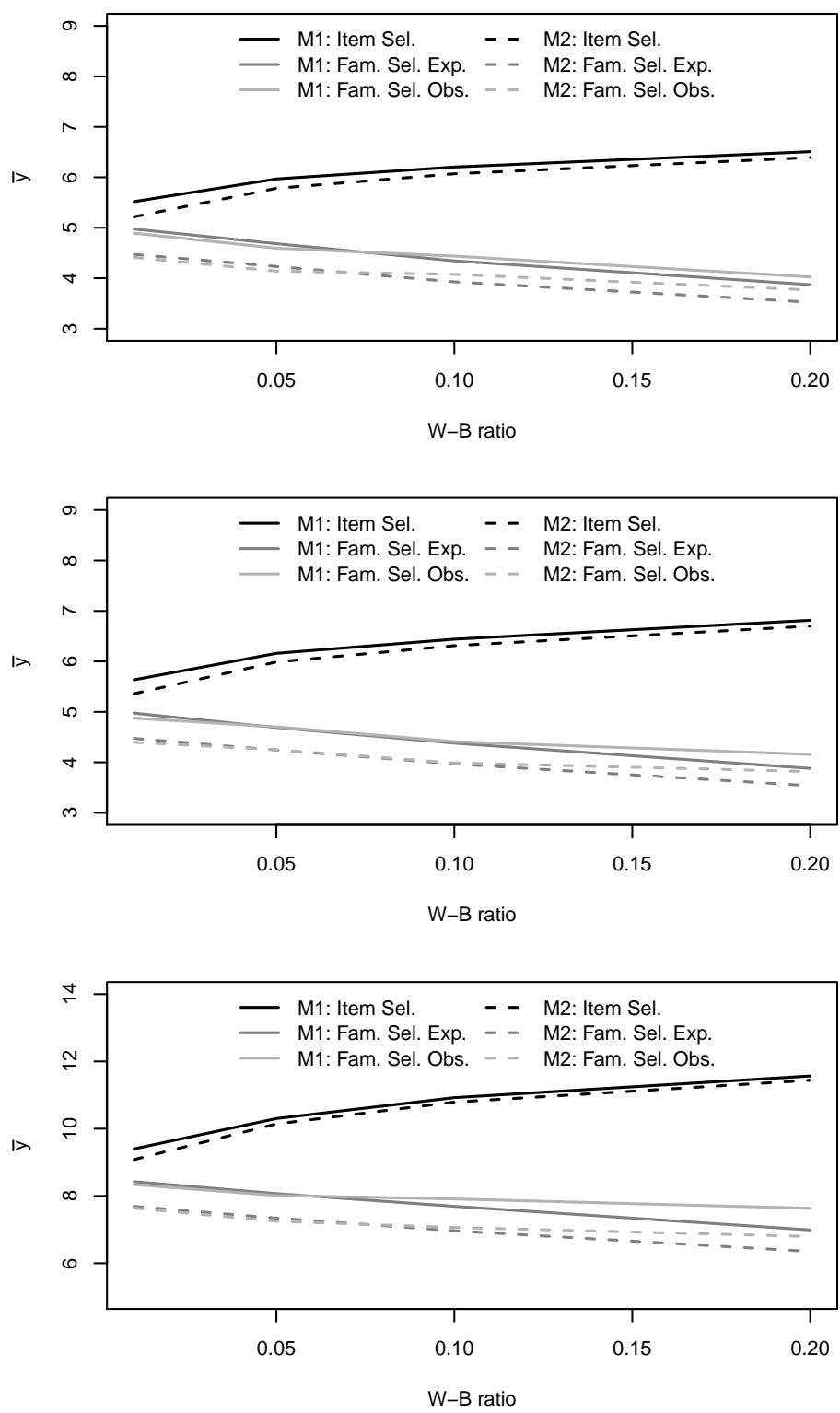

Figure 5.3: Mean value of the objective function $(\bar{y})$ for models M1 and M2 with a uniform relative target and with item selection and family selection, as a function of the ratio of within to between item parameter variability ( $\mathrm{W}$-B ratio). The upper plot is for the condition with $l=10$ selected families and $I_{f}=10$ items per family in the simulated item pool, the middle plot for 10 families and 20 items per family, and the lower plot for 20 families and 10 items per family. 

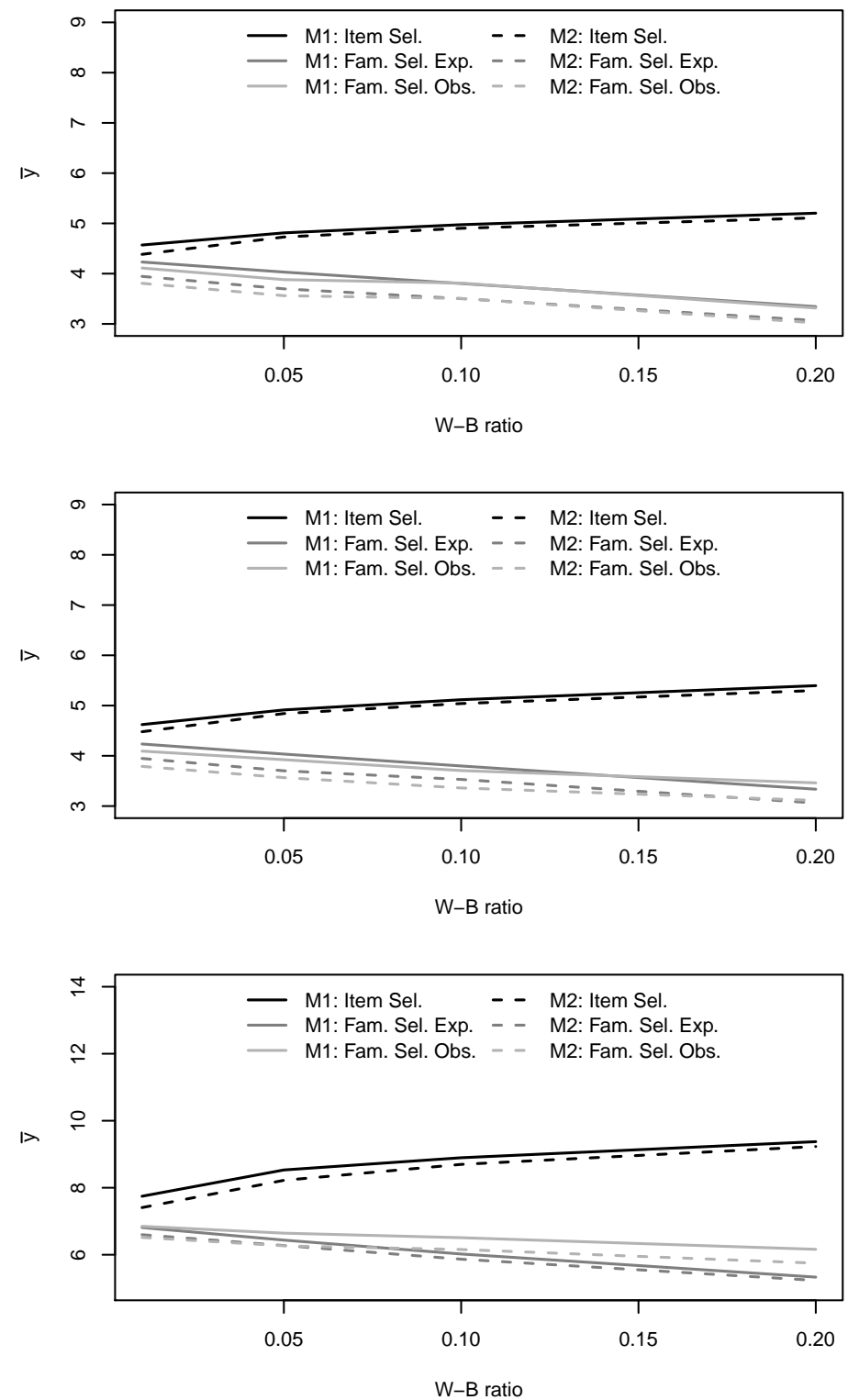

Figure 5.4: Mean value of the objective function $(\bar{y})$ for models M1 and M2 with a relative target with twice as much information at $\theta=0$ than at $\theta=-1$ and $\theta=1$ and with item selection and family selection, as a function of the ratio of within to between item parameter variability ( $\mathrm{W}-\mathrm{B}$ ratio). The upper plot is for the condition with $l=10$ selected families and $I_{f}=10$ items per family in the simulated item pool, the middle plot for 10 families and 20 items per family, and the lower plot for 20 families and 10 items per family. 

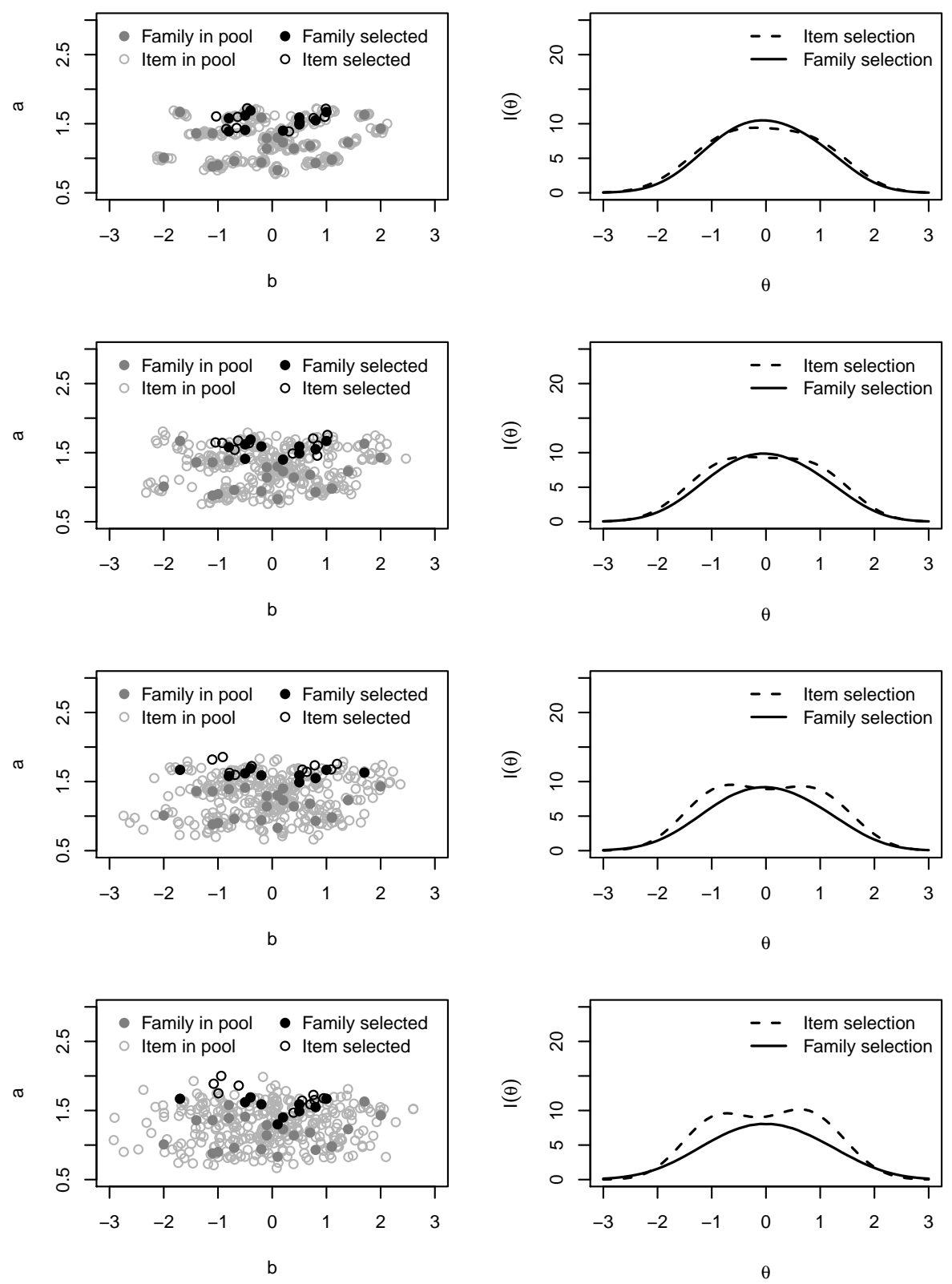

Figure 5.5: Simulated family and item parameters (left column) and family and item information curves (right column) for within-between covariance ratios of 0.01 (upper row), 0.05 (second row), 0.10 (third row), and 0.20 (last row), for a test selected based on a uniform relative target. 

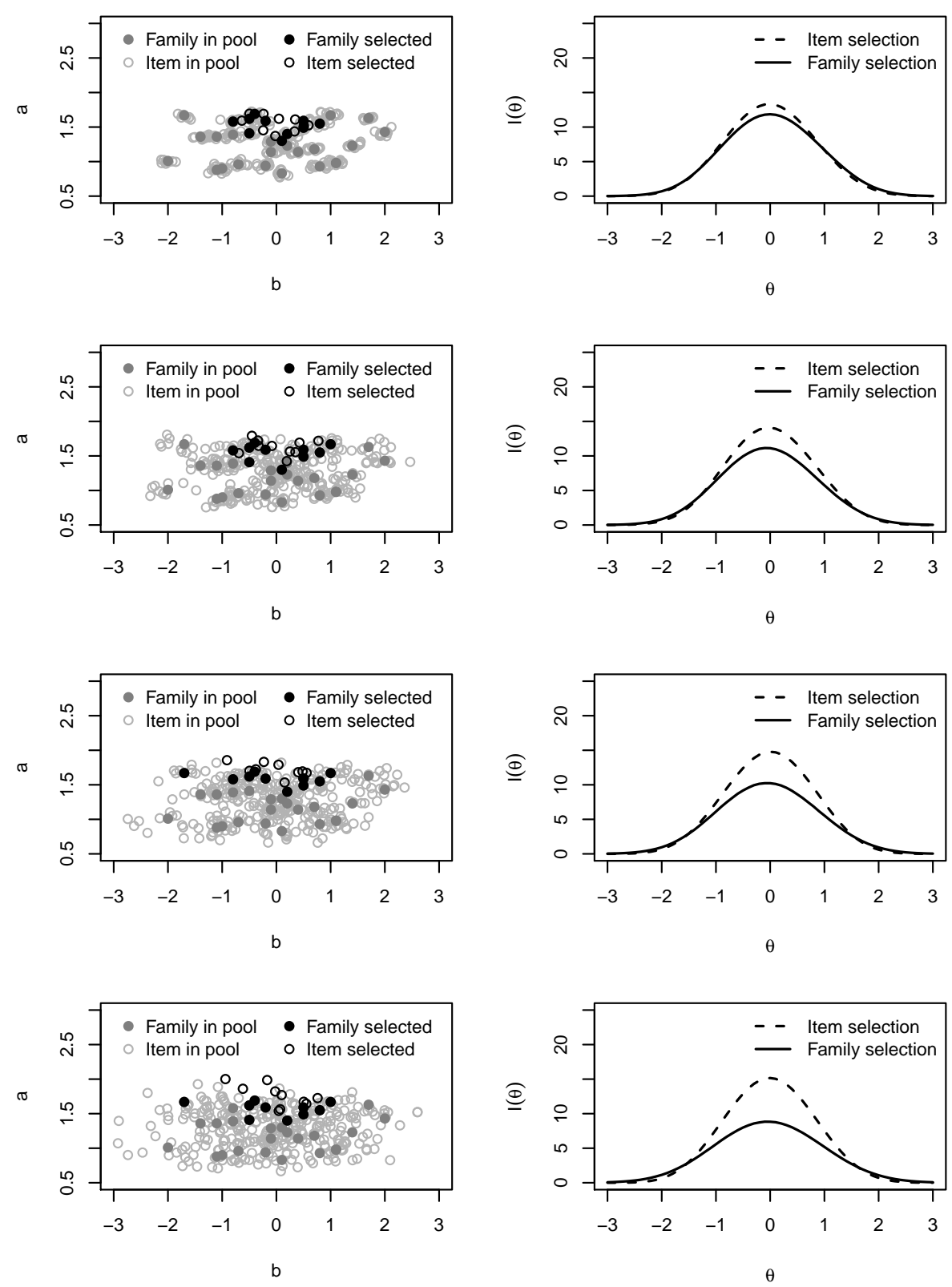

Figure 5.6: Simulated family and item parameters (left column) and family and item information curves (right column) for within-between covariance ratios of 0.01 (upper row), 0.05 (second row), 0.10 (third row), and 0.20 (last row), for a test selected based on a relative target with twice as much information at $\theta=0$ than at $\theta=-1$ and $\theta=1$. 
typically want both: For families with small item-parameter variability, ability can be estimated with larger precision (Glas \& van der Linden, 2003). However, items from such families may be more easily detected by test takers to be essentially the same. In other words, we should strike a careful balance between the wish to reduce estimation error and the danger of family disclosure.

In general, the Fisher information measure in (5.6) prefers families with low over high variability (but also takes the covariance between their item parameters into account). To mitigate the effects of low variability on family disclosure, an exposure-control method could be used; that is, with increasing exposure of the family over time, it could be given a lower weight in the test-assembly process. 


\section{Appendices}





\section{Appendix A}

\section{Models and Gibbs Sampling Algorithms}

The models used in this thesis are of two general types. The first type assumes identical item parameters for cloned items (i.e., items from the same family), whereas the second type allows for deviations of individual item parameters from their family means. In the literature, the models are known as identical siblings models (Johnson \& Sinharay, 2005) and item cloning models (Glas \& van der Linden, 2003) or related siblings models (Johnson \& Sinharay, 2005), respectively. In this thesis, both types of models are based on the three-parameter normal ogive model,

$$
p\left(U_{i_{f} n}=1 \mid \theta_{n}, a_{i_{f}}, b_{i_{f}}, \gamma_{i_{f}}\right)=\gamma_{i_{f}}+\left(1-\gamma_{i_{f}}\right) \Phi\left(a_{i_{f}} \theta_{n}-b_{i_{f}}\right),
$$

where $U_{i_{f} n}$ is a dichotomous variable for the response of person $n$ to item $i_{f}$. Furthermore, $\theta_{n}$ is the ability parameter of person $n, \boldsymbol{\xi}_{i_{f}}=\left(a_{i_{f}}, b_{i_{f}}, \gamma_{i_{f}}\right)$ are the item discrimination, difficulty, and guessing parameters of item $i_{f}, i_{f}=1, \ldots, I_{f}$, in family $f, f=1, \ldots, F$, and $\Phi($.$) is the cumulative normal distribution. The$ two-parameter normal ogive model is obtained by setting the guessing parameters to zero.

In the identical siblings models, the item parameters in (A.1) equal their respective family parameters $\boldsymbol{\mu}_{f}=\left(\mu_{a_{f}}, \mu_{b_{f}}, \mu_{\gamma_{f}}\right)$, which may in turn be explained by a set of effect parameters $\boldsymbol{\lambda}=\left(\boldsymbol{\lambda}^{a}, \boldsymbol{\lambda}^{b}, \boldsymbol{\lambda}^{\gamma}\right)$ :

$$
\begin{aligned}
\boldsymbol{\xi}_{i_{f}} & =\boldsymbol{\mu}_{f}, \\
\mu_{a_{f}} & =\boldsymbol{X}_{f}^{a} \boldsymbol{\lambda}^{a}, \\
\mu_{b_{f}} & =\boldsymbol{X}_{f}^{b} \boldsymbol{\lambda}^{b}, \\
\mu_{\gamma_{f}} & =\boldsymbol{X}_{f}^{\gamma} \boldsymbol{\lambda}^{\gamma} .
\end{aligned}
$$

The matrices $\boldsymbol{X}^{a}=\left(\boldsymbol{X}_{f}^{a}\right), \boldsymbol{X}^{b}=\left(\boldsymbol{X}_{f}^{b}\right)$, and $\boldsymbol{X}^{\gamma}=\left(\boldsymbol{X}_{f}^{\gamma}\right)$ describe the relationship between the family and effect parameters, and determine the exact model.

In the item cloning models, the item parameters are transformed as $\boldsymbol{\xi}_{i_{f}}=$ 
$\left(a_{i_{f}}, b_{i_{f}}, c_{i_{f}}=\operatorname{logit} \gamma_{i_{f}}\right)$ and are modeled by multivariate normal distributions:

$$
\begin{aligned}
\boldsymbol{\xi}_{i_{f}} & \sim \operatorname{MVN}\left(\boldsymbol{\mu}_{f}, \boldsymbol{\Sigma}_{f}\right), \\
\mu_{a_{f}} & =\boldsymbol{X}_{f}^{a} \boldsymbol{\lambda}^{a} \\
\mu_{b_{f}} & =\boldsymbol{X}_{f}^{b} \boldsymbol{\lambda}^{b} \\
\mu_{c_{f}} & =\boldsymbol{X}_{f}^{c} \boldsymbol{\lambda}^{c} .
\end{aligned}
$$

Analogous to (A.3)-(A.5), the matrices $\boldsymbol{X}^{a}, \boldsymbol{X}^{b}$, and $\boldsymbol{X}^{c}=\left(\boldsymbol{X}_{f}^{c}\right)$ in (A.7)-(A.9) determine the model, and $\boldsymbol{\lambda}=\left(\boldsymbol{\lambda}^{a}, \boldsymbol{\lambda}^{b}, \boldsymbol{\lambda}^{c}\right)$ are the effect parameters. The main differences between the two types of models are the covariance matrices $\boldsymbol{\Sigma}_{f}$, for $f=1, \ldots, F$, which describe the variability of the item parameters within families; compare (A.2) and (A.6).

Examples of types of matrices were given in Figures 4.2 and 4.3 for the eight families of statistical word problems. The first type, $\boldsymbol{D}_{1}$, is an identity matrix, and represents the case of a unique mean parameter for every family. Matrices $\boldsymbol{D}_{2}$ and $\boldsymbol{D}_{3}$ are design matrices, with and without interaction effects, respectively, and represent cases in which the family mean parameters are explained by radicals. The last matrix, $\boldsymbol{D}_{5}$, is an array of ones, and represents a common family mean parameter for all items. As examples, for the item cloning model (pp. 16 and 17) $\boldsymbol{X}^{a}, \boldsymbol{X}^{b}$ and $\boldsymbol{X}^{c}$ are all of matrix type $\boldsymbol{D}_{1}$, whereas for the linear item cloning model (same pages) $\boldsymbol{X}^{b}$ is of type $\boldsymbol{D}_{2}$ or $\boldsymbol{D}_{3}$. Note that other models can be defined, simply by changing $\boldsymbol{X}^{a}$ and $\boldsymbol{X}^{b}$ (see, for example, Table 4.1), and in case of guessing parameters in an item cloning model also $\boldsymbol{X}^{c}$. As $\boldsymbol{\lambda}^{\gamma}$ in the identical siblings model is the only element of $\boldsymbol{\lambda}$ that is not estimated by linear regression, we fix $\boldsymbol{X}^{\gamma}$ at an identity matrix. That is, for these models we assume that guessing parameters are either not included in the model, or family-specific.

\section{A.1 Identical Siblings Models}

Albert (1992) devised a Gibbs sampling scheme to estimate the parameters of the 2PNO model. In order to be able to obtain simple conditional distributions, he augmented the observed data with latent data (Tanner, 1996). An extension to the 3PNO model was suggested in Johnson and Albert (1999, section 6.9; see also Béguin \& Glas, 2001). The suggestion is based on the assumption that person $n$ knows the correct answer to item $i_{f}$ with probability $\Phi\left(a_{i_{f}} \theta_{n}-b_{i_{f}}\right)$ and then gives a correct response with probability one. Alternatively, the person does not know the answer with probability $\left(1-\Phi\left(a_{i_{f}} \theta_{n}-b_{i_{f}}\right)\right)$ and then guesses the correct answer with probability $\gamma_{i_{f}}$. The marginal probability of a correct response is the sum of the probabilities associated with these two processes,

$$
p\left(U_{i_{f} n}=1 \mid \theta_{n}, \boldsymbol{\xi}_{i_{f}}\right)=\Phi\left(a_{i_{f}} \theta_{n}-b_{i_{f}}\right)+\gamma_{i_{f}}\left(1-\Phi\left(a_{i_{f}} \theta_{n}-b_{i_{f}}\right)\right) .
$$

This interpretation suggests the introduction of latent augmentation variable $W_{i_{f} n}$,

$W_{i_{f} n}=1$ if person $n$ knows the correct answer to item $i_{f}$,

$W_{i_{f} n}=0 \quad$ if person $n$ does not know the correct answer to item $i_{f}$. 
The conditional probability of $W_{i_{f} n}$ given response $U_{i_{f} n}$ is given by

$$
\begin{aligned}
& p\left(W_{i_{f} n}=1 \mid U_{i_{f} n}=1, \theta_{n}, \boldsymbol{\xi}_{i_{f}}\right) \propto \Phi\left(a_{i_{f}} \theta_{n}-b_{i_{f}}\right), \\
& p\left(W_{i_{f} n}=0 \mid U_{i_{f} n}=1, \theta_{n}, \boldsymbol{\xi}_{i_{f}}\right) \propto \gamma_{i_{f}}\left(1-\Phi\left(a_{i_{f}} \theta_{n}-b_{i_{f}}\right)\right), \\
& p\left(W_{i_{f} n}=1 \mid U_{i_{f} n}=0, \theta_{n}, \boldsymbol{\xi}_{i_{f}}\right)=0, \\
& p\left(W_{i_{f} n}=0 \mid U_{i_{f} n}=0, \theta_{n}, \boldsymbol{\xi}_{i_{f}}\right)=1 .
\end{aligned}
$$

If a person gives an incorrect response to an item, (s)he is assumed not to know the answer; otherwise it would have been given.

Let $\boldsymbol{\delta}_{i_{f}}=\left(a_{i_{f}}, b_{i_{f}}\right)$. A second augmentation variable, $Z_{i_{f} n}$, is defined conditionally on $w_{i_{f} n}$ as a truncated normally distributed variable (Albert, 1992):

$$
\begin{aligned}
Z_{i_{f} n} \mid w_{i_{f} n}, \theta_{n}, \boldsymbol{\delta}_{i_{f}} & \sim N\left(a_{i_{f}} \theta_{n}-b_{i_{f}}, 1\right), \\
Z_{i_{f} n}<0 & \text { if } \quad W_{i_{f} n}=0 \\
Z_{i_{f} n} \geq 0 & \text { if } \quad W_{i_{f} n}=1 .
\end{aligned}
$$

In terms of the interpretation given above, if person $n$ does not know the answer to item $i_{f}$ the augmentation variable $Z_{i_{f} n}$ will be negative. Otherwise, it will be positive.

Because of these two types of data augmentation, the conditional posterior distributions of the ability and item discrimination and difficulty factor parameters can be obtained through a standard result from linear regression theory, which shows that the conditional posterior distributions of $\theta_{n}$ and $\boldsymbol{\lambda}=\left(\boldsymbol{\lambda}^{a}, \boldsymbol{\lambda}^{b}\right)$ are (multivariate) normal. The regression equation for $\theta_{n}$ is $\boldsymbol{z}_{n}+\boldsymbol{b}=\boldsymbol{a} \theta_{n}+\boldsymbol{\epsilon}_{n}$, and results in the following conditional distribution when using the prior $p\left(\theta_{n}\right) \sim$ $N(0,1)$ :

$$
\theta_{n} \mid \boldsymbol{z}_{n}, \boldsymbol{\delta} \sim N\left(\frac{\sum_{f=1}^{F} \sum_{i_{f}=1}^{I_{f}} a_{i_{f}}\left(z_{i_{f} n}+b_{i_{f}}\right)}{1+\sum_{f=1}^{F} \sum_{i_{f}=1}^{I_{f}} a_{i_{f}}^{2}},\left(1+\sum_{f=1}^{F} \sum_{i_{f}=1}^{I_{f}} a_{i_{f}}^{2}\right)^{-1}\right) .
$$

The regression equations for $\boldsymbol{\lambda}$ are

$$
\boldsymbol{z}_{i_{f}}=\boldsymbol{Q}_{f} \boldsymbol{\lambda}+\epsilon_{i_{f}}=\left[\boldsymbol{X}_{f}^{a} \boldsymbol{\theta},-\boldsymbol{X}_{f}^{b} \mathbf{1}_{N}\right]\left[\begin{array}{c}
\boldsymbol{\lambda}^{a} \\
\boldsymbol{\lambda}^{b}
\end{array}\right]+\epsilon_{i_{f}} .
$$

Using these equations, the discrimination and difficulty parameters $\boldsymbol{\lambda}$ can be sampled from the following conditional distribution:

$$
\boldsymbol{\lambda} \mid \boldsymbol{Q}, \boldsymbol{z} \sim M V N\left(\hat{\boldsymbol{\lambda}},\left(\boldsymbol{V}_{0}^{-1}+\sum_{f=1}^{F} I_{f}\left(\boldsymbol{Q}_{f}^{T} \boldsymbol{Q}_{f}\right)\right)^{-1}\right)
$$

where

$$
\hat{\boldsymbol{\lambda}}=\left(\boldsymbol{V}_{0}^{-1}+\sum_{f=1}^{F} I_{f}\left(\boldsymbol{Q}_{f}^{T} \boldsymbol{Q}_{f}\right)\right)^{-1}\left(\boldsymbol{V}_{0}^{-1} \boldsymbol{\lambda}_{0}+\sum_{f=1}^{F} \sum_{i_{f}=1}^{I_{f}} \boldsymbol{Q}_{f}^{T} \boldsymbol{z}_{i_{f}}\right)
$$


is the least squares estimator, and $\boldsymbol{\lambda}_{0}, \boldsymbol{V}_{0}$ are the mean and covariance matrix of the multivariate normal prior. For a noninformative prior, $\boldsymbol{\lambda}_{0}$ and $\boldsymbol{V}_{0}$ should be removed from (A.15). The draws for the item parameters $\boldsymbol{a}$ and $\boldsymbol{b}$ are computed from (A.2)-(A.4).

Let the number of persons that guess on the items from family $f$ and the number of persons that guess correctly on these items be given by

$$
\begin{aligned}
t_{f} & =\sum_{n=1}^{N} \sum_{i_{f}}^{I_{f}} I\left(w_{i_{f} n}=0\right), \\
s_{f} & =\sum_{n=1}^{N} \sum_{i_{f}}^{I_{f}} I\left(w_{i_{f} n}=0\right) u_{i_{f} n},
\end{aligned}
$$

respectively. The number of correct guesses, $s_{f}$, has a binomial distribution with parameters $t_{f}$ and $\gamma_{f}$. The conjugate prior for this conditional likelihood is the beta distribution with parameters $\alpha_{0}$ and $\beta_{0}$. The conditional posterior distribution of the guessing parameters is

$$
\gamma_{f} \mid \boldsymbol{u}_{f}, \boldsymbol{w}_{f} \sim \operatorname{Beta}\left(\alpha_{0}+s_{f}, \beta_{0}+t_{f}-s_{f}\right) .
$$

Since $\operatorname{Beta}(1,1)$ is the Uniform $(0,1)$ distribution, setting $\alpha_{0}=1$ and $\beta_{0}=1$ results in a noninformative prior.

In case of a model without guessing parameters, the draws for $\boldsymbol{W}$ (A.11) and $\gamma$ (A.18) are omitted, and the augmentation variable $\boldsymbol{W}$ in (A.12) is replaced by the data variable $\boldsymbol{U}$.

\section{A.2 Item Cloning Models}

The data augmentation variables $W_{i_{f} n}$ and $Z_{i_{f} n}$, and the ability parameters $\theta_{n}$ are drawn from (A.11)-(A.13), respectively. The discrimination and difficulty parameters $\boldsymbol{\delta}=(\boldsymbol{a}, \boldsymbol{b})$ are sampled using the linear regression models $\boldsymbol{z}_{i_{f}}=$ $\left[\boldsymbol{\theta},-\mathbf{1}_{N}\right] \boldsymbol{\delta}_{i_{f}}+\boldsymbol{\epsilon}_{i_{f}}$. Let $\boldsymbol{X}=\left(\boldsymbol{\theta},-\mathbf{1}_{N}\right)$, then

$$
\boldsymbol{\delta}_{i_{f}} \mid \boldsymbol{z}_{i_{f}}, \boldsymbol{\theta}, \boldsymbol{\mu}_{\delta_{f}}, \boldsymbol{\Sigma}_{\delta_{f}} \sim M V N\left(\hat{\boldsymbol{\delta}}_{i_{f}},\left(\boldsymbol{\Sigma}_{\delta_{f}}^{-1}+\boldsymbol{X}^{T} \boldsymbol{X}\right)^{-1}\right)
$$

with

$$
\hat{\boldsymbol{\delta}}_{i_{f}}=\left(\boldsymbol{\Sigma}_{\delta_{f}}^{-1}+\boldsymbol{X}^{T} \boldsymbol{X}\right)^{-1}\left(\boldsymbol{\mu}_{\delta_{f}} \boldsymbol{\Sigma}_{\delta_{f}}^{-1}+\boldsymbol{X}^{T} \boldsymbol{z}_{i_{f}}\right),
$$

where $\boldsymbol{\mu}_{\delta_{f}}$ and $\boldsymbol{\Sigma}_{\delta_{f}}$ are the elements of $\boldsymbol{\mu}_{f}$ and $\boldsymbol{\Sigma}_{f}$ corresponding to the discrimination and difficulty parameters. Note that $\hat{\boldsymbol{\delta}}_{i_{f}}$ is a precision weighted estimate composed of the mean parameters $\boldsymbol{\mu}_{\delta_{f}}$ and $\boldsymbol{\Sigma}_{\delta_{f}}$ and an item-specific effect, which is the classical least-squares estimate. The prior causes the item parameter estimates to 'shrink' towards their mean values. The restriction of positive discrimination parameters can be applied by redrawing when the restriction is violated.

Guessing parameters $\gamma_{i_{f}}$ are obtained in a separate step, conditional on $\boldsymbol{W}_{i_{f}}$, $\boldsymbol{U}_{i_{f}}$, and the draws of the second-level parameters. Let $t_{i_{f}}$ be the number of 
persons who do not know the correct answer to item $i_{f}$ and guess the response. The number of correct guesses, $s_{i_{f}}$, has a binomial distribution with parameters $t_{i_{f}}$ and $\gamma_{i_{f}}$. We assume that $c_{i_{f}}=\operatorname{logit}\left(\gamma_{i_{f}}\right)$ has a normal prior with parameters $\mu_{c_{f} \mid \delta_{f}}$ and $\Sigma_{c_{f} \mid \delta_{f}}$. Combining a binomial likelihood with this normal prior results in a non-standard posterior distribution but importance sampling can be used to obtain draws from it (Gelman et al., 2004).

When larger numbers of items per family are administered, family-specific covariance matrices can be estimated. Using the conjugate inverse-Wishart prior the conditional posterior distribution of the matrices is equal to

$$
\boldsymbol{\Sigma}_{f} \mid \boldsymbol{\xi}_{f}, \boldsymbol{\mu}_{f} \sim \text { inverse-Wishart }\left(\nu_{0}+I_{f},\left(\boldsymbol{S}_{0}+\boldsymbol{S}_{f}\right)^{-1}\right),
$$

with scale matrix $\boldsymbol{S}_{f}$ defined as

$$
\boldsymbol{S}_{f}=\sum_{i_{f}=1}^{I_{f}}\left(\boldsymbol{\xi}_{i_{f}}-\boldsymbol{\mu}_{f}\right)\left(\boldsymbol{\xi}_{i_{f}}-\boldsymbol{\mu}_{f}\right)^{T} .
$$

However, estimation of family-specific covariance matrices may lead to estimates with large standard errors when the number of items per family is small. As an alternative, it then makes sense to assume equal covariance structures across families, and estimate their common covariance matrix as

$$
\boldsymbol{\Sigma} \mid \boldsymbol{\xi}, \boldsymbol{\mu} \sim \text { inverse-Wishart }\left(\nu_{0}+\sum_{f=1}^{F} I_{f},\left(\boldsymbol{S}_{0}+\boldsymbol{S}\right)^{-1}\right),
$$

with

$$
\boldsymbol{S}=\sum_{f=1}^{F} \sum_{i_{f}=1}^{I_{f}}\left(\boldsymbol{\xi}_{i_{f}}-\boldsymbol{\mu}_{f}\right)\left(\boldsymbol{\xi}_{i_{f}}-\boldsymbol{\mu}_{f}\right)^{T},
$$

where $\boldsymbol{S}_{0}$ and $\nu_{0}$ are the sum of squares matrix and degrees of freedom of the inverse-Wishart prior.

The effect parameters $\boldsymbol{\lambda}=\left(\boldsymbol{\lambda}^{a}, \boldsymbol{\lambda}^{b}, \boldsymbol{\lambda}^{c}\right)$ are estimated using multivariate linear regression. The regression equation for item $i_{f}$ is

$$
\boldsymbol{\xi}_{i_{f}}=\boldsymbol{Q}_{f} \boldsymbol{\lambda}+\epsilon_{i_{f}}=\left[\begin{array}{ccc}
\boldsymbol{X}_{f}^{a} & \mathbf{0} & \mathbf{0} \\
\mathbf{0} & \boldsymbol{X}_{f}^{b} & \mathbf{0} \\
\mathbf{0} & \mathbf{0} & \boldsymbol{X}_{f}^{c}
\end{array}\right]\left[\begin{array}{c}
\boldsymbol{\lambda}^{a} \\
\boldsymbol{\lambda}^{b} \\
\boldsymbol{\lambda}^{c}
\end{array}\right]+\epsilon_{i_{f}},
$$

where $\epsilon_{i_{f}}$ is our generic notation for a vector of normally distributed error terms with mean zero and covariance matrix $\boldsymbol{\Sigma}_{f}$.

The equation in (A.22) is an example of what Griffiths and Valenzuela (2006) have called a set of "seemingly unrelated regressions." The term was coined by Zellner (1962) and refers to regression models in which each dependent variable is related to a possibly different set of explanatory variables. For correlated dependent variables, Zellner (1962) shows that the use of generalized least squares results in more efficient estimation of the regression coefficients than ordinary least squares. 
Using the conjugate multivariate normal prior the conditional posterior distribution of $\boldsymbol{\lambda}$ is

$$
\boldsymbol{\lambda} \mid \boldsymbol{Q}, \boldsymbol{\Sigma}, \boldsymbol{\xi} \sim M V N\left(\hat{\boldsymbol{\lambda}},\left(\boldsymbol{V}_{0}^{-1}+\sum_{f=1}^{F} I_{f}\left(\boldsymbol{Q}_{f}^{T} \boldsymbol{\Sigma}_{f}^{-1} \boldsymbol{Q}_{f}\right)\right)^{-1}\right)
$$

where

$$
\hat{\boldsymbol{\lambda}}=\left(\boldsymbol{V}_{0}^{-1}+\sum_{f=1}^{F} I_{f}\left(\boldsymbol{Q}_{f}^{T} \boldsymbol{\Sigma}_{f}^{-1} \boldsymbol{Q}_{f}\right)\right)^{-1}\left(\boldsymbol{V}_{0}^{-1} \boldsymbol{\lambda}_{0}+\sum_{f=1}^{F} \sum_{i_{f}=1}^{I_{f}} \boldsymbol{Q}_{f}^{T} \boldsymbol{\Sigma}_{f}^{-1} \boldsymbol{\xi}_{i_{f}}\right)
$$

is the generalized least squares estimator. The draws for the family mean parameters $\boldsymbol{\mu}_{a}, \boldsymbol{\mu}_{b}$ and $\boldsymbol{\mu}_{c}$ are computed as (A.7)-(A.9), respectively.

In case of a model without guessing parameters, the draws for $\boldsymbol{W}$ (A.11) and $\gamma$ are omitted, and the augmentation variable $\boldsymbol{W}$ in (A.12) is replaced by the data variable $\boldsymbol{U}$. Furthermore, $\boldsymbol{Q}_{f}$ and $\boldsymbol{\lambda}$ in (A.22) are redefined to exclude the elements corresponding to the guessing parameters. 


\section{Appendix B}

\section{Family Information}

Family information can be defined as the expected information in the response to a random item from a family. The computations required to obtain values for the family information function depend on the type of model used; the type of identical siblings models or that of item cloning models (see Appendix A).

\section{B.1 Identical Siblings Models}

Family information for the general class of identical siblings models in (A.1)-(A.5) is given by

$$
I_{f}(\theta)=-\mathrm{E}_{u}\left[\frac{\partial^{2}}{\partial \theta^{2}} \ln p\left(u \mid \theta, \boldsymbol{\mu}_{f}\right)\right] .
$$

Let $P_{u}=p\left(u \mid \theta, \boldsymbol{\mu}_{f}\right)$ be the likelihood of the model, and let $P_{1}$ and $P_{0}$ be the probabilities of a correct and incorrect response according to this model, respectively. Furthermore, let $P_{1}^{\prime}$ be the first derivative of $P_{1}$ with respect to the ability parameter $\theta$ (see Table B.1, second row). Because for this class of models the item parameters $\boldsymbol{\xi}_{i_{f}}$ are restricted to be equal to their respective family parameters $\boldsymbol{\mu}_{f}$, family information can simply be computed from the well-known item information formula (Birnbaum, 1968) with the family parameters substituted for the item parameters; that is,

$$
I_{f}(\theta)=\frac{\left[P_{1}^{\prime}\right]^{2}}{P_{1} P_{0}}
$$

\section{B.2 Item Cloning Models}

Family information for the general class of item cloning models in (A.1), (A.6)(A.9), is given by

$$
I_{f}(\theta)=-\mathrm{E}_{u}\left[\frac{\partial^{2}}{\partial \theta^{2}} \ln \int p\left(u \mid \theta, \boldsymbol{\xi}_{i_{f}}\right) p\left(\boldsymbol{\xi}_{i_{f}} \mid \boldsymbol{\mu}_{f}, \boldsymbol{\Sigma}_{f}\right) d \boldsymbol{\xi}_{i_{f}}\right] .
$$


Let $\int \ldots d G=\int \ldots p\left(\boldsymbol{\xi}_{i_{f}} \mid \boldsymbol{\mu}_{f}, \boldsymbol{\Sigma}_{f}\right) d \boldsymbol{\xi}_{i_{f}}$, so that (B.3) can be abbreviated as

$$
I_{f}(\theta)=-\mathrm{E}_{u}\left[\frac{\partial^{2}}{\partial \theta^{2}} \ln \int p\left(u \mid \theta, \boldsymbol{\xi}_{i_{f}}\right) d G\right] .
$$

Let $L_{u}=\int p\left(u \mid \theta, \boldsymbol{\xi}_{i_{f}}\right) d G$ denote the likelihood of the hierarchical model (the ICM or LICM), and let $L_{1}=\int p\left(U=1 \mid \theta, \boldsymbol{\xi}_{i_{f}}\right) d G$ and $L_{0}=\int p\left(U=0 \mid \theta, \boldsymbol{\xi}_{i_{f}}\right) d G$ denote the probabilities of a correct and incorrect response, respectively, according to this model. Family information is then derived as

$$
\begin{aligned}
I_{f}(\theta) & =-\mathrm{E}_{u}\left[\frac{\partial^{2}}{\partial \theta^{2}} \ln L_{u}\right] \\
& =-\mathrm{E}_{u}\left[\frac{\partial}{\partial \theta} \frac{L_{u}^{\prime}}{L_{u}}\right], \\
& =-\mathrm{E}_{u}\left[\frac{L_{u}^{\prime \prime} L_{u}-L_{u}^{\prime} L_{u}^{\prime}}{L_{u}^{2}}\right]=-\mathrm{E}_{u}\left[\frac{L_{u}^{\prime \prime}}{L_{u}}-\left(\frac{L_{u}^{\prime}}{L_{u}}\right)^{2}\right] .
\end{aligned}
$$

Let $P_{u}=p\left(u_{f} \mid \theta, \boldsymbol{\xi}_{i_{f}}\right)$ be the likelihood of the first-level model, and let $P_{1}$ and $P_{0}$ be the probabilities of a correct and incorrect response according to this model, respectively. Making use of Leibniz' rule for changing the order of integration and differentiation (e.g., Casella \& Berger, 2002, section 2.4), the first and second derivatives of $L_{1}$ and $L_{0}$ can be obtained by Monte Carlo integration over the first and second derivatives of $P_{1}$ and $P_{0}$, respectively:

$$
\begin{aligned}
L_{1}^{\prime} & =\frac{\partial}{\partial \theta} \int P_{1} d G=\int \frac{\partial P_{1}}{\partial \theta} d G, \\
L_{1}^{\prime \prime} & =\int \frac{\partial^{2} P_{1}}{\partial \theta^{2}} d G, \\
L_{0}^{\prime} & =\int \frac{\partial P_{0}}{\partial \theta} d G, \\
L_{0}^{\prime \prime} & =\int \frac{\partial^{2} P_{0}}{\partial \theta^{2}} d G .
\end{aligned}
$$

The expectation in (B.5) can thus be rewritten as

$$
\begin{aligned}
I_{f}(\theta)= & -\mathrm{E}_{u}\left[\frac{\frac{\partial^{2}}{\partial \theta^{2}} \int P_{u} d G}{\int P_{u} d G}-\left(\frac{\frac{\partial}{\partial \theta} \int P_{u} d G}{\int P_{u} d G}\right)^{2}\right], \\
= & -\left[\int \frac{\partial^{2}}{\partial \theta^{2}}\left[P_{1}\right] d G-\frac{\left(\int \frac{\partial}{\partial \theta}\left[P_{1}\right] d G\right)^{2}}{\int P_{1} d G}\right] \\
& -\left[\int \frac{\partial^{2}}{\partial \theta^{2}}\left[P_{0}\right] d G-\frac{\left(\int \frac{\partial}{\partial \theta}\left[P_{0}\right] d G\right)^{2}}{\int P_{0} d G}\right] .
\end{aligned}
$$

The first and second derivatives of $P_{1}$ and $P_{0}$ are well-known and easy to compute (see Table B.1). 
Table B.1: Probability of a correct response $\left(P_{1}\right)$ and its first $\left(P_{1}^{\prime}\right)$ and second $\left(P_{1}^{\prime \prime}\right)$ derivative with respect to $\theta$ for two normal ogive first-level models.

\begin{tabular}{lll}
\hline & $2 \mathrm{PNO}$ & $3 \mathrm{PNO}$ \\
\hline$P_{1}$ & $\Phi(a[\theta-b])$ & $c+(1-c) \Phi(a[\theta-b])$ \\
$P_{1}^{\prime}$ & $a \phi(a[\theta-b])$ & $(1-c) a \phi(a[\theta-b])$ \\
$P_{1}^{\prime \prime}$ & $-a^{2}(a[\theta-b]) \phi(a[\theta-b])$ & $-(1-c) a^{2}(a[\theta-b]) \phi(a[\theta-b])$ \\
\hline
\end{tabular}

The first and second derivative of the probability of an incorrect response equal the respective derivatives for a correct response, with a reversed sign. If the alternative parameterization with $a \theta-b$ as the argument of $\Phi($.$) is used,$ as in Appendix A, the square brackets should be removed from the formulas.

Table B.2: Standard deviation of the computed family information for the $2 \mathrm{PNO}$ and the $3 \mathrm{PNO}$ as a function of the number of Monte Carlo iterations.

\begin{tabular}{rcccccc}
\hline Number of & \multicolumn{3}{c}{$2 \mathrm{PNO}$} & \multicolumn{3}{c}{$3 \mathrm{PNO}$} \\
\cline { 2 - 7 } iterations & $\theta=-1$ & $\theta=0$ & $\theta=1$ & $\theta=-1$ & $\theta=0$ & $\theta=1$ \\
\hline 1000 & 0.006 & 0.011 & 0.009 & 0.003 & 0.007 & 0.005 \\
5000 & 0.003 & 0.005 & 0.003 & 0.002 & 0.003 & 0.002 \\
10,000 & 0.002 & 0.003 & 0.003 & 0.001 & 0.002 & 0.001 \\
20,000 & 0.001 & 0.002 & 0.002 & 0.001 & 0.002 & 0.001 \\
\hline
\end{tabular}

\section{Number of Monte Carlo Iterations}

A small simulation study was performed to investigate the required number of iterations to obtain desirable precision in the computed family information. Both the $2 \mathrm{PNO}$ and $3 \mathrm{PNO}$ models, parameterized with $a_{i_{f}}\left[\theta_{n}-b_{i_{f}}\right]$ as the argument of $\Phi($.$) , were used as first-level models. For the former, the mean discrimination$ and difficulty parameters per family were fixed at one and zero, respectively; for the latter, the mean discrimination and guessing parameters were fixed at one and 0.2 , while the mean difficulty was chosen to be optimal at $\theta=0$; i.e., $\mu_{b}=-0.258$ (see Wolfe, 1981). For all models, the variances and covariance of all parameters were set equal to 0.1 and 0.05 , respectively. Family information was computed 100 times at $\boldsymbol{\theta}=-1,0$, and 1 . The numbers of Monte Carlo iterations to compute the information was $1000,5000,10,000$, or 20,000 . The standard deviation over the replications was used as a measure of precision of the information estimates.

Table B.2 presents the results from the study. The larger values for the family information measure required more iterations to obtain the same precision; compare, for example, the differences between the standard deviations for the twoparameter and three-parameter models and those for $\theta=0$ and $\theta=\{-1,1\})$. In general however, the precision was high. In the main simulation studies of Chapter 5, 10,000 iterations were used to compute family information. As shown in Table B.2, for this number the standard error of the family information estimates for 
each of the models can be expected to be smaller than 0.005 . 


\section{References}

Albert, J. H. (1992). Bayesian estimation of normal-ogive item response curves using Gibbs sampling. Journal of Educational and Behavioral Statistics, 17, 261-269.

Andersen, E. B. (1972). The numerical solution of a set of conditional estimation equations. Journal of the Royal Statistical Society, Series B, 34, 42-54.

Arendasy, M., \& Sommer, M. (2005). The effect of different types of perceptual manipulations on the dimensionality of automatically generated figural matrices. Intelligence, 33, 307-324.

Arendasy, M., \& Sommer, M. (2007). Using psychometric technology in educational assessment: The case of a schema-based isomorphic approach to the automatic generation of quantitative reasoning items. Learning and Individual Differences, 17, 366-383.

Arendasy, M., Sommer, M., Gittler, G., \& Hergovich, A. (2006). Automatic generation of quantitative reasoning items: A pilot study. Journal of Individual Differences, 27, 2-14.

Baker, F. B., \& Kim, S.-H. (2004). Item response theory: Parameter estimation techniques. New York: Marcel Dekker.

Béguin, A. A., \& Glas, C. A. W. (2001). MCMC estimation and some model-fit analysis of multidimensional IRT models. Psychometrika, 66, 541-562.

Bennett, R. E., Steffen, M., Singley, M. K., Morley, M., \& Jacquemin, D. (1997). Evaluating an automatically scorable, open-ended response type for measuring mathematical reasoning in computer-adaptive tests. Journal of Educational Measurement, 34, 162-176.

Birnbaum, A. (1968). Some latent-trait models and their use in inferring an examinee's ability. In F. M. Lord \& M. R. Novick (Eds.), Statistical theories of mental test scores (pp. 397-479). Reading, MA: Addison-Wesley.

Boer Rookhuiszen, R. (2011). Generation of German narrative probability exercises. Unpublished master's thesis, Enschede, The Netherlands: University of Twente.

Bormuth, J. R. (1970). On the theory of achievement test items. Chicago, IL: University of Chicago Press.

Bradlow, E. T., Wainer, H., \& Wang, X. (1999). A Bayesian random effects model for testlets. Psychometrika, 64, 153-168.

Casella, G., \& Berger, R. L. (2002). Statistical inference. USA: Duxbury.

Casella, G., \& George, E. I. (1992). Explaining the Gibbs sampler. The American Statistician, 46, 167-174. 
Chaloner, K., \& Brant, R. (1988). A Bayesian approach to outlier detection and residual analysis. Biometrika, 75, 651-659.

Chapelle, C. A., \& Chung, Y.-R. (2010). The promise of NLP and speech processing technologies in language assessment. Language Testing, 27, 301-315.

Cho, S.-J., \& Rabe-Hesketh, S. (2011). Alternating imputation posterior estimation of models with crossed random effects. Computational Statistics and Data Analysis, 55, 12-25.

De Boeck, P. (2008). Random item IRT models. Psychometrika, 73, 533-559.

De Boeck, P., \& Wilson, M. (2004). Explanatory item response models: A generalized linear and nonlinear approach. New York: Springer.

Embretson, S. E. (1998). A cognitive design system approach to generating valid tests: Application to abstract reasoning. Psychological Methods, 3, 380-396.

Embretson, S. E. (1999). Generating items during testing: Psychometric issues and models. Psychometrika, 64, 407-433.

Embretson, S. E. (2005). Measuring human intelligence with artificial intelligence: Adaptive item generation. In R. J. Sternberg \& J. E. Pretz (Eds.), Cognition and intelligence: Identifying the mechanisms of the mind (pp. 251-267). Cambridge, UK: Cambridge University Press.

Embretson, S. E., \& Reise, S. (2000). Item response theory for psychologists. Mahwah, NJ: Erlbaum.

Enright, M. K., Morley, M., \& Sheehan, K. M. (2002). Items by design: The impact of systematic feature variation on item statistical characteristics. Applied Measurement in Education, 15, 49-74.

Ferguson, R. W. (2000). Modeling orientation effects in symmetry detection: The role of visual structure. In L. R. Gleitman \& A. K. Joshi (Eds.), Proceedings of the twenty-second annual conference of the cognitive science society (pp. 125-130). Hillsdale, USA: Lawrence Erlbaum Associates.

Fischer, G. H. (1973). The linear logistic test model as an instrument in educational research. Acta Psychologica, 37, 359-374.

Fox, J.-P. (2004). Multilevel IRT model assessment. In L. A. van der Ark, M. A. Croon, \& K. Sijtsma (Eds.), New developments in categorical data analysis for the social and behavioral sciences (pp. 227-252). London: Lawrence Erlbaum Associates.

Fox, J.-P., \& Glas, C. A. W. (2001). Bayesian estimation of a multilevel IRT model using Gibbs sampling. Psychometrika, 66, 271-288.

Freund, P. A., Hofer, S., \& Holling, H. (2008). Explaining and controlling for the psychometric properties of computer-generated figural matrix items. Applied Psychological Measurement, 32, 195-210.

Geerlings, H., Glas, C. A. W., \& van der Linden, W. J. (2011). Modeling rule-based item generation. Psychometrika, 76, 337-359.

Gelman, A., Carlin, J. B., Stern, H. S., \& Rubin, D. B. (2004). Bayesian data analysis. USA: Chapman \& Hall.

Gelman, A., Meng, X.-L., \& Stern, H. (1996). Posterior predictive assessment of model fitness via realized discrepancies. Statistica Sinica, 6, 733-807.

Gelman, A., \& Pardoe, I. (2006). Bayesian measures of explained variance and pooling in multilevel (hierarchical) models. Technometrics, 48, 241-251.

Gelman, A., Van Mechelen, I., Verbeke, G., Heitjan, D. F., \& Meulders, M. (2005). 
Multiple imputation for model checking: Complete-data plots with missing and latent data. Biometrics, 61, 74-85.

Geweke, J. (1992). Evaluating the accuracy of sampling-based approaches to the calculation of posterior moments. In J. M. Bernardo, J. O. Berger, A. P. Dawid, \& A. F. M. Smith (Eds.), Bayesian statistics 4: Proceedings of the fourth valencia international meeting (pp. 169-193). Oxford: Oxford University Press.

Gilbride, T. J., \& Lenk, P. L. (2010). Posterior predictive model checking: An application to multivariate normal heterogeneity. Journal of Marketing Research, 47, 896-909.

Glas, C. A. W. (2010). Item parameter estimation and item fit analysis. In W. J. van der Linden \& C. A. W. Glas (Eds.), Elements of adaptive testing (pp. 269-288). New York, NJ: Springer.

Glas, C. A. W., \& Geerlings, H. (2009). Psychometric aspects of pupil monitoring systems. Studies in Educational Evaluation, 35, 83-88.

Glas, C. A. W., \& Meijer, R. R. (2003). A Bayesian approach to person fit analysis in item response theory models. Applied Psychological Measurement, 27, 217-233.

Glas, C. A. W., \& van der Linden, W. J. (2001). Modeling variability in item parameters in item response models (Research Rep. 01-11). Enschede, The Netherlands: University of Twente.

Glas, C. A. W., \& van der Linden, W. J. (2003). Computerized adaptive testing with item cloning. Applied Psychological Measurement, 27, 247-261.

Glas, C. A. W., van der Linden, W. J., \& Geerlings, H. (2010). Estimation of the parameters in an item-cloning model for adaptive testing. In W. J. van der Linden \& C. A. W. Glas (Eds.), Elements of adaptive testing (pp. 289-314). New York, NJ: Springer.

Gorin, J. S. (2005). Manipulating processing difficulty of reading comprehension questions: The feasibility of verbal item generation. Journal of Educational Measurement, 42, 351-373.

Gorin, J. S., \& Embretson, S. E. (2006). Item difficulty modeling of paragraph comprehension items. Applied Psychological Measurement, 30, 394-411.

Goto, T., Kojiri, T., Watanabe, T., Iwata, T., \& Yamada, T. (2010). Automatic generation system of multiple-choice cloze questions and its evaluation. Knowledge Management \& E-Learning: An International Journal, 2, 210-224.

Green, K. E., \& Smith, R. M. (1987). A comparison of two methods of decomposing item difficulties. Journal of Educational and Behavioral Statistics, 12, 369381.

Griffiths, W. E., \& Valenzuela, M. R. (2006). Gibbs samplers for a set of seemingly unrelated regressions. Australian and New Zealand Journal of Statistics, 48 , 335-351.

Hambleton, R. K., \& Jones, R. W. (1994). Item parameter estimation errors and their influence on test information functions. Applied Measurement in Education, 7, 171-186.

Hambleton, R. K., Jones, R. W., \& Rogers, H. J. (1993). Influence of item parameter estimation errors in test development. Journal of Educational 
Measurement, 30, 143-155.

He, Q., \& Veldkamp, B. P. (2010, July). Including unstructured textual information in CAT prior selection. Presentation at the International Meeting of the Psychometric Society 2010, Athens, Georgia, USA.

Heidelberger, P., \& Welch, P. D. (1983). Simulation run length control in the presence of an initial transient. Operations Research, 31, 1109-1144.

Hively, W., Patterson, H. L., \& Page, S. H. (1968). A "universe-defined" system of arithmetic achievement items. Journal of Educational Measurement, 5, $275-290$.

Hofer, S. (2004). MatrixDeveloper [unpublished computer software]. Münster, Germany: Psychological Institute IV, Westfälische Wilhelms-Universität.

Holling, H., Bertling, J. P., \& Zeuch, N. (2009). Automatic item generation of probability word problems. Studies in Educational Evaluation, 35, 71-76.

Holling, H., Blank, H., Kuchenbäcker, K., \& Kuhn, J.-T. (2008). Rule-based item design of statistical word problems: A review and first implementation. Psychology Science Quarterly, 50, 363-378.

Hornke, L. F., \& Habon, M. W. (1986). Rule-based item bank construction and evaluation within the linear logistic framework. Applied Psychological Measurement, 10, 369-380.

ILOG, I. (2003). CPLEX 9.0 [Computer program]. Incline Village, NV: Author.

Irvine, S. H. (2002). The foundations of item generation for mass testing. In S. H. Irvine \& P. C. Kyllonen (Eds.), Item generation for test development (pp. 3-34). Mahwah, NJ: Lawrence Erlbaum Associates.

Irvine, S. H., \& Kyllonen, P. C. (Eds.). (2002). Item generation for test development. Mahwah, NJ: Lawrence Erlbaum Associates.

Janssen, R., Schepers, J., \& Peres, D. (2004). Models with item and item group predictors. In P. De Boeck \& M. Wilson (Eds.), Explanatory item response models: A generalized linear and nonlinear approach (pp. 189-212). New York: Springer.

Janssen, R., Tuerlinckx, F., Meulders, M., \& De Boeck, P. (2000). A hierarchical IRT model for criterion-referenced measurement. Journal of Educational and Behavioral Statistics, 25, 285-306.

Jodoin, M. G. (2003). Measurement efficiency of innovative item formats in computer-based testing. Journal of Educational Measurement, 40, 1-15.

Johnson, M. S., \& Sinharay, S. (2005). Calibration of polytomous item families using Bayesian hierarchical modeling. Applied Psychological Measurement, 29, 369-400.

Johnson, V. E., \& Albert, J. H. (1999). Ordinal data modeling. New York: Springer.

Karamanis, N., Ha, L. A., \& Mitkov, R. (2006). Generating multiple-choice test items from medical text: A pilot study. In Proceedings of the fourth international natural language generation conference (pp. 104-107). Stroudsburg, PA, USA: The Association for Computational Linguistics.

Klein Entink, R. H., Fox, J.-P., \& van der Linden, W. J. (2009). A multivariate multilevel approach to the modeling of accuracy and speed of test takers. Psychometrika, 74, 21-48.

Klein Entink, R. H., Kuhn, J.-T., Hornke, L. F., \& Fox, J.-P. (2009). Evaluating 
cognitive theory: A joint modeling approach using responses and response times. Psychological Methods, 14, 54-75.

Klein Entink, R. H., van der Linden, W. J., \& Fox, J.-P. (2009). A Box-Cox normal model for response times. British Journal of Mathematical and Statistical Psychology, 62, 621-640.

Kohs, S. C. (1923). Intelligence measurement. New York: MacMillan Press.

Kubinger, K. D. (2008). On the revival of the Rasch model-based LLTM: From constructing tests using item generating rules to measuring item administration effects. Psychology Science Quarterly, 50, 311-327.

Lane, S. (1991). Use of restricted item response models for examining item difficulty ordering and slope uniformity. Journal of Educational Measurement, 28, 295-309.

Laros, J. A., \& Tellegen, P. J. (1991). Construction and validation of the SON-R 5.5-17, the Snijders-Oomen non-verbal intelligence test. Groningen: WoltersNoordhoff.

Lee, J., \& Corter, J. E. (2011). Diagnosis of subtraction bugs using Bayesian networks. Applied Psychological Measurement, 35, 27-47.

Lewis, C. (2001). Expected response functions. In A. Boomsma, M. A. J. van Duijn, \& T. A. B. Snijders (Eds.), Essays on item reponse theory (pp. 163171). New York: Springer.

Liu, C.-L., Wang, C.-H., \& Gao, Z.-M. (2005). Using lexical constraints to enhance the quality of computer-generated multiple-choice cloze items. Computational Linguistics and Chinese Language Processing, 10, 303-328.

Lord, F. M., \& Novick, M. R. (1968). Statistical theories of mental test scores. Reading, MA: Addison-Wesley.

Luecht, R. M. (2009, June). Adaptive computer-based tasks under an assessment engineering paradigm. Paper presented at the 2009 Graduate Management Admission Council Conference on Computerized Adaptive Testing, Minneapolis, Minnesota, USA.

MacEachern, S. N., \& Berliner, L. M. (1994). Subsampling the Gibbs sampler. The American Statistician, 48, 188-190.

Matteucci, M., Mignani, S., \& Veldkamp, B. P. (2010). On using item features to estimate parameters in IRT models. Presentation at the Joint Meeting GfKI-CLADAG 2010 in Firenze, Italy.

Miller, J. C., \& Skillman, G. D. (2008). Relationship of stimulus and examinee variables to performance on analogous visual and tactile block construction tasks. Applied Neuropsychology, 15, 140-149.

Millman, J., \& Westman, R. S. (1989). Computer-assisted writing of achievement test items: Toward a future technology. Journal of Educational Measurement, 26, 177-190.

Mislevy, R. J., \& Levy, R. (2007). Bayesian psychometric modeling from an evidence-centered design perspective. In C. R. Rao \& S. Sinharay (Eds.), Handbook of statistics, volume 26 (pp. 839-865). North-Holland: Elsevier.

Mitkov, R., Ha, L. A., \& Karamanis, N. (2006). A computer-aided environment for generating multiple-choice test items. Natural Language Engineering, 12, $177-194$.

Mostow, J., Beck, J. E., Bey, J., Cuneo, A., Sison, J., Tobin, B., et al. (2004). 
Using automated questions to assess reading comprehension, vocabulary, and effects of tutorial interventions. Technology, Instruction, Cognition and Learning, 2, 97-134.

Osburn, H. G. (1968). Item sampling for achievement testing. Educational and Psychological Measurement, 28, 95-104.

Plummer, M., Best, N., Cowles, K., \& Vines, K. (2006). CODA: Convergence diagnosis and output analysis for MCMC. $R$ News, $6,7-11$. Available from http://CRAN.R-project.org/doc/Rnews/

R Development Core Team. (2009). R: A language and environment for statistical computing [Computer software manual]. Vienna, Austria. Available from http://www.R-project.org

Raftery, A. E., \& Lewis, S. (1992). How many iterations in the Gibbs sampler? In J.-M. Bernardo, J. O. Berger, A. P. Dawid, \& A. F. M. Smith (Eds.), Bayesian statistics 4: Proceedings of the fourth valencia international meeting (pp. 763-773). Oxford: Oxford University Press.

Rasch, G. (1960). Probabilistic models for some intelligence and attainment tests. Copenhagen: Danish Institute for Educational Research.

Reed, S. K. (1987). A structure-mapping model for word problems. Journal of Experimental Psychology: Learning, Memory and Cognition, 13, 124-139.

Reiter, E., \& Dale, R. (2000). Building natural language generation systems. New York: Cambridge University Press.

Rijmen, F., \& De Boeck, P. (2002). The random weights linear logistic test model. Applied Psychological Measurement, 26, 271-285.

Rijmen, F., Tuerlinckx, F., De Boeck, P., \& Kuppens, P. (2003). A nonlinear mixed model framework for item response theory. Psychological Methods, 8, 185-205.

Roid, G., \& Haladyna, T. (1982). A technology for test-item writing. New York: Academic Press.

Rubin, D. B. (1976). Inference and missing data. Biometrika, 63, 581-592.

Sinharay, S., \& Johnson, M. S. (2008). Use of item models in a large-scale admissions test: A case study. International Journal of Testing, 8, 209-236.

Sinharay, S., Johnson, M. S., \& Stern, H. S. (2006). Posterior predictive assessment of item response theory models. Applied Psychological Measurement, 30 , $298-321$.

Sinharay, S., Johnson, M. S., \& Williamson, D. M. (2003). Calibrating item families and summarizing the results using family expected response functions. Journal of Educational and Behavioral Statistics, 28, 295-313.

Sireci, S. G., \& Zenisky, A. L. (2006). Innovative item formats in computer-based testing: In pursuit of improved construct representation. In S. M. Downing \& T. M. Haladyna (Eds.), Handbook of test development (pp. 329-347). Mahwah, NJ: Lawrence Erlbaum Associates.

Smith, S., Avinesh, P. V. S., \& Kilgarriff, A. (2010). Gap-fill tests for language learners: Corpus-driven item generation. In Proceedings of icon-2010: 8th international conference on natural language processing. India: Macmillan Publishers.

Sonnleitner, P. (2008). Using the LLTM to evaluate an item-generating system for reading comprehension. Psychology Science Quarterly, 50, 345-362. 
Spiegelhalter, D. J., Best, N. G., Carlin, B. P., \& van der Linde, A. (2002). Bayesian measures of model complexity and fit. Journal of the Royal Statistical Society B, 64, 583-639.

Steinbakk, G. H., \& Storvik, G. O. (2009). Posterior predictive p-values in Bayesian hierarchical models. Scandinavian Journal of Statistics, 36, 320336.

Sumita, E., Sugaya, F., \& Yamamoto, S. (2005). Measuring non-native speakers' proficiency of English by using a test with automatically-generated fill-inthe-blank questions. In J. Burstein \& C. Leacock (Eds.), Proceedings of the second workshop on building educational applications using NLP (pp. 61-68). USA: Association for Computational Linguistics.

Tanner, M. A. (1996). Tools for statistical inference: Methods for the exploration of posterior distributions and likelihood functions. New York: Springer-Verlag.

Tatsuoka, K. K. (1983). Rule space: An approach for dealing with misconceptions based on item response theory. Journal of Educational Measurement, 20, $345-354$.

Tellegen, P. J., \& Laros, J. A. (1993). The construction and validation of a nonverbal test of intelligence: The revision of the Snijders-Oomen tests. European Journal of Psychological Assessment, 9, 147-157.

Theune, M., Boer Rookhuiszen, R., op den Akker, R., \& Geerlings, H. (2011). Generating varied narrative probability exercises. In Proceedings of the sixth workshop on innovative use of NLP for building educational applications (pp. 20-29). Portland, Oregon: Association for Computational Linguistics.

Timms, M. J. (2007). Using item response theory (IRT) to select hints in an ITS. In R. Luckin, K. R. Koedinger, \& J. Greer (Eds.), Proceedings of the 2007 conference on artificial intelligence in education: Building technology rich learning contexts that work (pp. 213-221). Amsterdam, The Netherlands: IOS Press.

Tsutakawa, R. K., \& Johnson, J. C. (1990). The effect of uncertainty of item parameter estimation on ability estimates. Psychometrika, 55, 371-390.

Van den Noortgate, W., De Boeck, P., \& Meulders, M. (2003). Cross-classification multilevel logistic models in psychometrics. Journal of Educational and Behavioral Statistics, 28, 369-386.

van der Kleij, F. M., Timmers, C. F., \& Eggen, T. J. H. M. (2011). The effectiveness of methods for providing written feedback through a computer-based assessment for learning: A systematic review. CADMO, 21-38.

van der Linden, W. J. (Ed.). (2005). Linear models for optimal test design. New York: Springer.

van der Linden, W. J., \& Glas, C. A. W. (2000). Capitalization on item calibration error in adaptive testing. Applied Measurement in Education, 13, 35-53.

van der Linden, W. J., \& Glas, C. A. W. (2001). Cross-validating item parameter estimation in adaptive testing. In A. Boomsma, M. A. J. van Duijn, \& T. A. B. Snijders (Eds.), Essays on item reponse theory (pp. 205-219). New York: Springer.

van der Linden, W. J., \& Glas, C. A. W. (2010). Elements of adaptive testing. New York: Springer.

van der Linden, W. J., \& Hambleton, R. K. (Eds.). (1997). Handbook of modern 
item response theory. New York: Springer.

van der Linden, W. J., Klein Entink, R. H., \& Fox, J.-P. (2010). IRT parameter estimation with response times as collateral information. Applied Psychological Measurement, 34, 327-347.

van der Linden, W. J., \& Pashley, P. J. (2010). Item selection and ability estimation in adaptive testing. In W. J. van der Linden \& C. A. W. Glas (Eds.), Elements of adaptive testing (pp. 3-30). New York: Springer.

Verguts, T., \& De Boeck, P. (2000). A Rasch model for detecting learning while solving an intelligence test. Applied Psychological Measurement, 24, 151162.

Verhelst, N. D., \& Glas, C. A. W. (1993). A dynamic generalization of the Rasch model. Psychometrika, 58, 395-415.

Verhelst, N. D., \& Glas, C. A. W. (1995). The one parameter logistic model. In G. H. Fischer \& I. W. Molenaar (Eds.), Rasch models: Foundations, recent developments, and applications (pp. 215-238). New York: Springer-Verlag.

Weiss, D. J., \& Kingsbury, G. G. (1984). Application of computerized adaptive testing to educational problems. Journal of Educational Measurement, 21, $361-375$.

Williams, H. P. (1993). Model solving in mathematical programming. Chichester, England: John Wiley \& Sons Ltd.

Wolfe, J. H. (1981). Optimal item difficulty for the three-parameter normal ogive response model. Psychometrika, 46, 461-464.

Xi, X. (2010). Automated scoring and feedback systems: Where are we and where are we heading? Language Testing, 27, 291-300.

Zellner, A. (1962). An efficient method of estimating seemingly unrelated regressions and tests for aggregation bias. Journal of the American Statistical Association, 57, 348-368.

Zeuch, N. (2011). Rule-based item construction: Analysis with and comparison of linear logistic test models and cognitive diagnostic models with two item types. Unpublished doctoral dissertation, University of Münster, Münster, Germany.

Zhang, J., Xie, M., Song, X., \& Lu, T. (2011). Investigating the impact of uncertainty about item parameters on ability estimation. Psychometrika, 76, 97-118. 


\section{Samenvatting}

In de toetspraktijk zijn vaak grote itembanken nodig om (meerdere) betrouwbare toetsen samen te kunnen stellen. Na een initiële investering in het maken van een item generator kan tijd bespaart worden door de items automatisch te laten genereren. Hierbij kan onderscheid gemaakt worden tussen constructieregels die de moeilijkheid van de items beïnvloeden en regels die alleen voor oppervlakkige variatie in item kenmerken zorgen. De eerste methode zorgt ervoor dat de bank items bevat met een brede spreiding in moeilijkheid, terwijl de tweede methode kan worden gebruikt om de itembank verder uit te breiden. De combinatie van de twee item generatie technieken leidt tot groepen van items ("item families") die vergelijkbare psychometrische eigenschappen hebben die te verklaren zijn aan de hand van de constructieregels. Dit geeft bijvoorbeeld de mogelijkheid om elke test afnemer een unieke toets aan te bieden die op een willekeurig tijdstip kan worden gemaakt, zonder dat daarmee de validiteit van de test in het geding komt.

In dit proefschrift worden item respons theorie (IRT) modellen besproken die gebruikt kunnen worden om de psychometrische eigenschappen van item families te schatten op basis van een steekproef van items. Er wordt onderscheid gemaakt tussen modellen die veronderstellen dat de parameters voor de items gelijk zijn aan hun familiegemiddelden en modellen die item-specifieke afwijkingen toestaan. Uitbreidingen van de modellen met verklarende variabelen voor de familiegemiddelden staan een onderzoek naar het effect van de constructieregels op de moeilijkheid van de items toe. Methoden worden besproken voor het schatten van de modelparameters en voor het toetsen van modelpassing aan de data. Tevens wordt beschreven hoe op basis van deze modellen nieuwe tests ontworpen kunnen worden die de verwachte informatie in de responsen op de automatisch genereerde items over de vaardigheid van (een groep van) personen maximaliseren.

In hoofdstuk 1 worden de elementen besproken die nodig zijn voor een geheel automatisch systeem voor toetsgeneratie en -afname. Het voorbereidende werk, zoals het opstellen van een moeilijkheidstheorie en daarop gebaseerde constructieregels, het schatten van modelparameters en het toetsen van modelpassing, wordt gevolgd door het implementeren van een systeem dat een test kan ontwerpen, de items kan genereren en afnemen, de responsen van de test afnemers kan analyseren en eventueel de resultaten kan terugkoppelen naar de test afnemers.

In de daaropvolgende hoofdstukken (hoofdstuk 2-4) worden de verschillende IRT modellen in detail beschreven. In hoofdstuk 2 wordt het hiërarchische model van Glas en van der Linden (2003), dat elke familie met een multivariaat normaal verdeling modelleert, uitgebreid met verklarende variabelen voor de fami- 
liemoeilijkheid. Het oorspronkelijke model en de uitbreiding worden vergeleken door middel van een empirische toepassing op intelligentie data. Tevens wordt de nauwkeurigheid waarmee de parameters met een Bayesiaans schattingsalgoritme kunnen worden teruggeschat getoetst met een simulatiestudie. In elk van de onderzochte condities is de variantie in de familie parameterschattingen het kleinst bij een combinatie van een groter aantal items en een kleiner aantal personen dat een item maakt. Het heeft dan ook de aanbeveling om het afnamedesign goed te overwegen, om met een zo klein mogelijke steekproef van personen zo nauwkeurig mogelijke schattingen te kunnen krijgen.

Het effect van het negeren van residuele variantie in de itemparameters op de schattingen van de model parameters wordt onderzocht in hoofdstuk 3. Een cumulatief normaal versie van Fischer's (1973) lineair logistische test model wordt vergeleken met een uitbreiding waarin de residuele variantie in de itemparameters wordt opgevangen in een covariantiematrix. De vergelijking wordt gemaakt in een empirische studie met twee subtests uit een intelligentietest alsmede door een simulatiestudie. De resultaten van de studies laten zien dat zowel bij hypothesetoetsing als vaardigheidsschatting beide modellen conservatievere resultaten geven bij een grotere mate van residuele variantie in de itemparameters.

In hoofdstuk 4 wordt besproken hoe de modelpassing van de hierboven beschreven modellen aan data kan worden getoetst. In een empirische studie wordt het model van Glas en van der Linden (2003) toegepast op data over statistische woordproblemen. Het model wordt vervolgens systematisch gerestricteerd door het implementeren van assumpties over de familiegemiddelden en de residuele variantie in de itemparameters. De modelpassing wordt getoetst door een vergelijking tussen de geobserveerde en onder het model gerepliceerde waarden van teststatistieken. Vervolgens worden de meest restrictieve assumpties die niet tot misfit leidden gebruikt om een relatief spaarzaam model te verkrijgen dat de data adequaat beschrijft.

Hoofdstuk 5 laat zien hoe de geschatte familieparameters gebruikt kunnen worden om nieuwe tests te ontwerpen. Hiertoe wordt voor de itemfamilies in de hiërarchische modellen een informatiefunctie gedefinieerd als de verwachte waarde in een respons op een random item uit de familie. De informatiefunctie geeft over het algemeen een lagere waarde bij een grotere mate van binnen-familie variabiliteit in de itemparameters. Op basis van de informatiefunctie en eventuele restricties op het gebruik van de constructieregels kunnen de families worden geselecteerd en de items worden gegenereerd. In een simulatiestudie wordt de informatie in een test verkregen uit een itembank waarvan de parameters van alle items zijn geschat, vergeleken met dezelfde bank voor het geval dat alleen de familieparameters bekend zouden zijn. In het eerste geval benadert de test-informatiefunctie het doel beter dan in het tweede geval. Voor praktische toepassingen dienen de eerder genoemde voordelen van automatische itemgeneratie dan ook tegen dit nadeel van minder informatie per afgenomen item te worden afgewogen.

Twee appendices, waarin de Bayesiaanse schattingsalgoritmen (Appendix A) en de berekening van familie-informatiefuncties (Appendix B) worden uitgewerkt beëindigen het proefschrift. 